\title{
INFLUENCE OF HIGH ASPECT RATIO NANOPARTICLE FILLER ADDITION ON \\ PIEZOELECTRIC NANOCOMPOSITES
}

\author{
A Thesis \\ presented to \\ the Faculty of California Polytechnic State University, \\ San Luis Obispo
}

\author{
In Partial Fulfillment \\ of the Requirements for the Degree \\ Master of Science in Polymers and Coatings
}

by

Jeremy Armas

December 2018 
(C) 2018

Jeremy Armas

ALL RIGHTS RESERVED 
COMMITTEE MEMBERSHIP

TITLE: Influence of High Aspect Ratio Nanoparticle Filler Addition on Piezoelectric Nanocomposites

AUTHOR: Jeremy Armas

DATE SUBMITTED: December 2018

COMMITTEE CHAIR: Raymond Fernando, Ph. D.

Professor, Department of Chemistry and

Biochemistry

COMMITTEE MEMBER: Thomas Yong-Jin Han, Ph. D.

Group Leader, Functional Materials Synthesis

and Integration Group

Lawrence Livermore National Laboratory

COMMITTEE MEMBER: Erik Sapper, Ph. D.

Assistant Professor, Department of Chemistry and

Biochemistry 


\begin{abstract}
Influence of High Aspect Ratio Nanoparticle Filler Addition on Piezoelectric Nanocomposites Jeremy Armas
\end{abstract}

Piezoelectric nanogenerators (PNGs) are a new class of energy harvesting materials that show potential as a direct energy source for low powered electronics. Recently, piezoelectric polymers have been utilized for PNG technology due to low toxicity, high flexibility, and facile solution processing which provide manufacturing opportunities such as screen printing. Throughout the last decade, countless projects have focused on how to enhance the energy harvesting capabilities of these PNGs through the incorporation of nanoparticle fillers, which have been reported to enhance the piezoelectric properties of the film either directly through their intrinsic piezoelectric properties or through acting as surfaces for the interfacial nucleation of piezoelectric polymer crystals.

Herein, two systems of PNGs formed from piezoelectric copolymers poly(vinylidene fluoride-co-hexafluropropylene) or poly(vinylidene fluoride-co-trifluoroethylene) mixed with high aspect ratio zinc oxide nanowires, hydroxyl functionalized multi-walled carbon nanotubes, or carboxylic acid functionalized single walled carbon nanotubes were investigated. Variations of filler type and loading are tested to determine influences on film morphology and piezoelectric properties. Power harvesting tests are conducted to directly determine the effect of nanoparticle addition on the output power of the non-poled devices. Both copolymer systems are found to exhibit a non-linear increase in output power with the increase of nanoparticle filler loading. The crystal polymorph properties of both systems are investigated by Fourier transform infrared spectroscopy. The microstructure of the poly(vinylidene fluoride-co-trifluoroethylene) films are further examined using X-ray diffraction, differential scanning calorimetry, polarized optical microscopy, and atomic force microscopy to determine the mechanism behind the increased power harvesting capabilities. As well, explanations for perceived output power from "self-poled" films are briefly explored. 


\section{ACKNOWLEDGMENTS}

I would like to thank Dr. Thomas Yong-Jin Han at Lawrence Livermore National Laboratory for his mentorship throughout my research project. With his support I learned a great amount about project management and the importance of being a critically thinking researcher.

I would like to thank the members of my project team and the Functional Materials Synthesis and Integration group for help with planning, running experiments, and providing feedback for my project. A special thanks to Logan Bekker for training, Caitlyn Cook for project guidance, Tim Yee for SEM, and James Cahill for XRD testing. To have the opportunity to work alongside my peers at the lab has been a truly humbling and inspiring experience.

Finally, I would like to thank Dr. Raymond Fernando, Dr. Erik Sapper, Dr. Shanju Zhang, and other professors associated with the Polymers and Coatings program who have provided me with guidance not only during this project, but throughout my time as a Master's student in the Polymers and Coatings program.

This work was performed under the auspices of the U.S. Department of Energy by Lawrence Livermore National Laboratory. 


\section{TABLE OF CONTENTS}

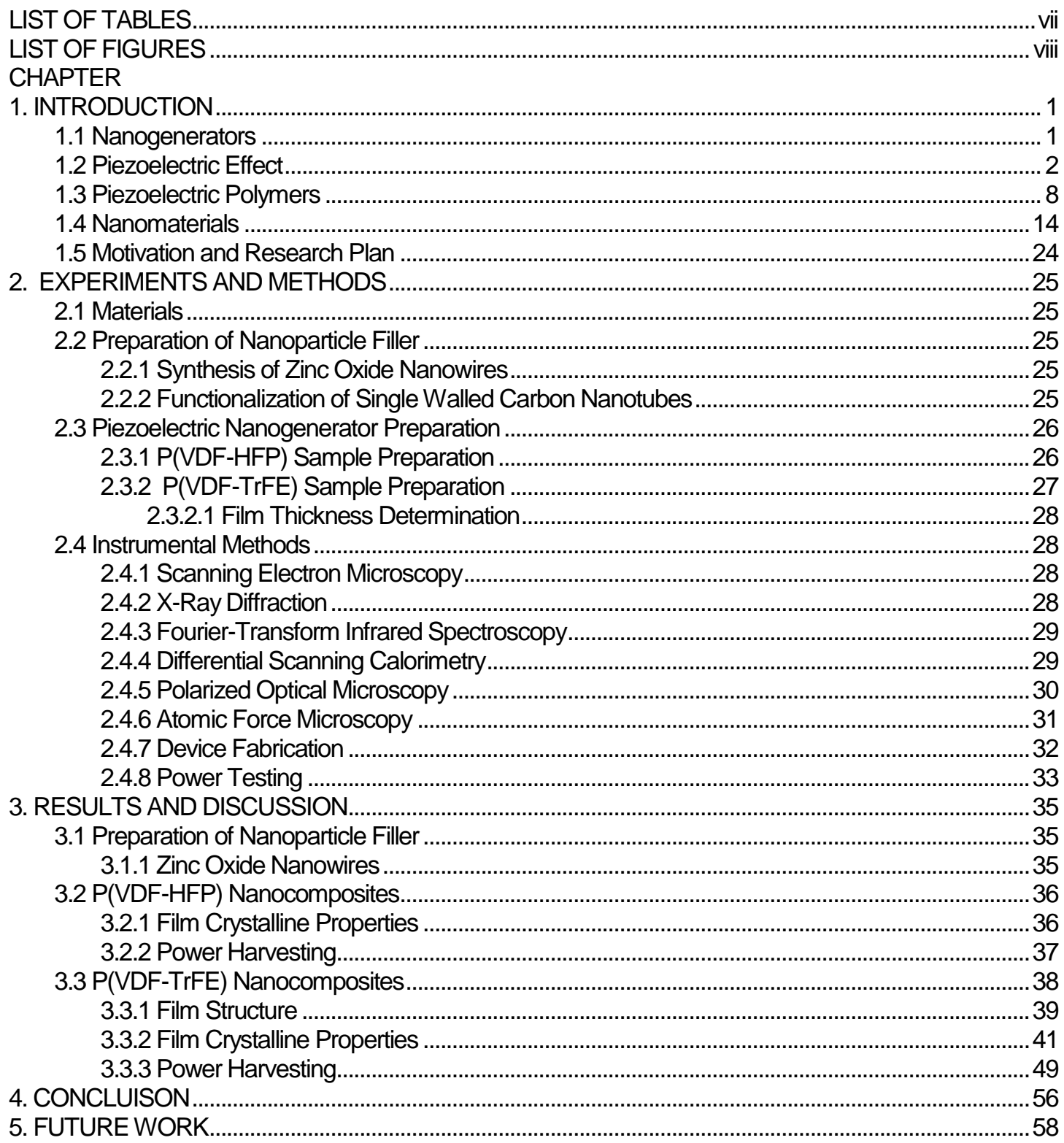

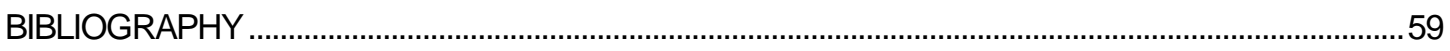

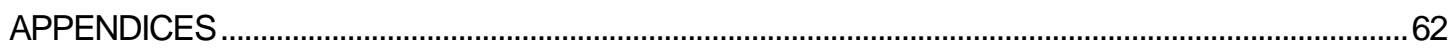

A. Supporting Information 62 


\section{LIST OF TABLES}

Table

Page

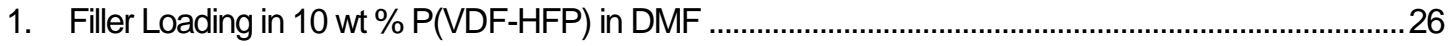

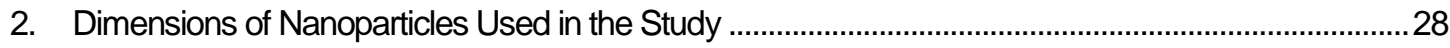

3. RMS Surface Roughness of Polymer Nanocomposite AFM Topographs .......................................... 48 


\section{LIST OF FIGURES}

Figure

1. Noncentrosymmetric perovskite unit cell of lead zirconate titanate with charge polarization across the structure below $T_{\text {curie }}$

2. (a) Unpoled piezoelectric with randomly oriented ferroelectric domains, (b) during poling, an external voltage is applied to the material to align ferroelectric domains, (c) after poling, domains are oriented in similar directions, resulting in a remnant polarization $\left(P_{r}\right)$ in the direction of the applied field. 4

3. Typical ferroelectric (P-E) hysteresis loop. ${ }^{1}$ 5

4. Generation of current across electrodes as a piezoelectric material is compressed and decompressed 6

5. General diagram of piezoelectric transducer with directional axis 1, 2, and 3 and shear axis 4,5 , and 6 7

6. Facile solution-based processing of polymer electric components gives rise to opportunities for a new class of flexible and sustainable polymer electronics

7. (Left) Different conformations in linear polymers (a) amorphous conformation (b) lamellar sheets (c) extended chain crystals (d) semi-crystalline matrix. (right) Illustration of polymer crystal lamellae.

8. (Left) Depiction of crystalline chain conformations of PVDF $\alpha / \delta$ and $\beta$ phases. (Right) Unit cells of the (a) $\alpha$-phase (b) $\delta$-phase (c) $\beta$-phase of PVDF projected parallel to chain axes. ${ }^{2}$

9. Schematic illustration showing (left to right) morphology of film after melt casting ( $\alpha$-phase), mechanical stretching to reorient chains to the extended $\beta$-phase, and application of external electric field to align polarization domains of crystals. 3 
10. Structure of PVDF copolymers (left) poly(vinylidene fluoride-cohexafluoropropylene) and (right) poly(vinylidene fluoride-cotrifluoroethylene)

11. Orientation of $\beta$-phase within the PVDF lamellar crystal throughout the $a, b$, and c axis. The (110) and (200) crystallographic planes shown in the upper left corner are commonly associated with the $\beta$-phase of (PVDF-TrFE) and are visible around $19.9^{\circ}$ using X-ray diffractometry. ${ }^{4}$

12. As the particle size within a constant volume decreases, the surface area to volume ratio increases significantly 14

13. Illustration of (a) Initial step deposition on substrate surface and (b) subsequent stem and fold formation resulting in development of crystal lamella. ${ }^{5}$ 17

14. Polarized optical microscopy images of (a) PVDF, (b) $0.1 \%$ CoFe2O4, and (c) $5 \%$ CoFe2O4 PNCs (200 $\mu \mathrm{m}$ scalebars in b and c). (d) Increase in $\beta$ phase in nanocomposites with increasing $\mathrm{CoFe} 2 \mathrm{O} 4$ nanocomposite sample measured through FTIR. ${ }^{6}$ 18

15. (a) Crystal structure of hexagonal wurtzite $\mathrm{ZnO}$ with a non-centrosymmetric unit cell (b) compression and extension of ions lead to changes in polarization which are stabilized by charge generation at electrode surfaces 20

16. (a) Lattice structure of carbon nanomaterials and (b) Structure of singlewalled (left) and multi-walled (right) carbon nanotube. ${ }^{51,52}$

17. Acid functionalization of carbon nanotubes which introduces polar groups to the surface of CNTs

18. Method for piezoelectric nanogenerator device fabrication. 32

19. (Top) circuit used for power harvesting of piezoelectric nanogenerator (PNG) (Bottom) Actual power harvesting circuit on breadboard 
20. SEM images of synthesized $\mathrm{ZnO} N W$, where images show (left) dispersion of $\mathrm{ZnO} \mathrm{NW}(x 5,500)$ and (right) zoomed in depiction of $\mathrm{ZnO} N W$ diameters $(x 90,000)$

21. XRD diffractogram of solvothermally grown $\mathrm{ZnO} N W$ confirming noncentrosymmetric hexagonal wurtzite structure with lattice parameters of $a=$ $3.25 \AA$ and $c=5.21 \AA$ 36

22. Results from FTIR study (a) FTIR spectra of $100 \mu \mathrm{m}$ wet cast (b) FTIR spectra of $200 \mu \mathrm{m}$ wet cast (c) Fraction of beta phase $F(\beta)$ of $P(V D F-H F P)$ copolymer composites with 1 wt $\%$ MWCNT-OH and a range of 0,4 , and 8 wt $\%$ ZnO NW casted at $100 \mu \mathrm{m}$ and $200 \mu \mathrm{m}$ wet films 37

23. Output voltage of $\mathrm{P}(\mathrm{VDF}-\mathrm{HFP})$ formulations from tapping demonstrating an increase in increase in voltage with the addition of nanoparticle filler 38

24. Film thickness of 100 and $200 \mu \mathrm{m}$ wet cast films with $0,0.1,0.5$, or 1 wt $\%$ nanofiller

25. $200 \mu \mathrm{m} P($ VDF-TrFE) films loaded with 1 wt \% SWCNT-COOH casted (a) before and (b) after 1 minute of pulsed probe sonication 40

26. FTIR spectra of $100 \mu \mathrm{m}$ wet cast samples with the addition of $0.1,0.5$, or 1 wt \% (left to right) ZnO NW, MWCNT-OH, or SWCNT-COOH 42

27. FTIR spectra of $200 \mu \mathrm{m}$ wet cast samples with the addition of $0.1,0.5$, or 1 wt \% (left to right) ZnO NW, MWCNT-OH, or SWCNT-COOH

28. XRD diffractogram of $\mathrm{P}(\mathrm{VDF}-\mathrm{TrFE})$ films with variable loading of nanoparticle filler 44

29. Percent crystallinity $\left(X_{c}\right)$ of polymer nanocomposites with varying loading of ZnO NW, MWCNT-OH, and SWCNT-COOH 45

30. Atomic force microscopy topograph of control $P(V D F-T r F E)$ film with no added filler 46 
31. Atomic force microscopy topographs of P(VDF-TrFE) PNCs with (left to right)

0.1 wt $\%, 0.5$ wt $\%$, and 1 wt $\% \mathrm{ZnO} N W$

32. Atomic force microscopy topographs of P(VDF-TrFE) PNCs with (left to right)

0.1 wt $\%, 0.5$ wt $\%$, and 1 wt $\%$ MWCNT-OH

33. Atomic force microscopy topographs of P(VDF-TrFE) PNCs with (left to right)

0.1 wt $\%, 0.5$ wt $\%$, and 1 wt $\%$ SWCNT-COOH

34. Output voltage of devices from Instron compression testing

35. Output current of devices from Instron compression testing 49

36. Device volume power density from Instron compression testing 50

37. Oscillatory compression test output voltage using $0.5 \mathrm{wt} \%$ SWCNT-COOH film over (left) multiple seconds and (right) a single compression/decompression cycle showing a $74 \mathrm{~ms}$ compressive signal 51

38. Output voltage from tapping SWCNT-COOH 0.5 (left) tapping over time showing sustained output voltage and (right) peak and trough outlined in left figure demonstrating typical time duration of compressive and decompressive voltage peaks 52

39. Output current from tapping SWCNT-COOH 0.5 tapping 52

40. Output voltage from tapping $0.1 \mathrm{wt} \% \mathrm{ZnO} N W$ PNG with (left) no rectification (right) with full wave bridge rectifier 53

41. Discharge of $10 \mathrm{nF}$ capacitor charged with 10 seconds moderate hand tapping used to power several green and yellow LED lights (inset)

42. Output voltage in the (left) forward and (right) reverse direction showing polarization-switching with the $0.5 \mathrm{wt} \%$ SWCNT-COOH film

43. Voltage generation through flicking (left) overview of signals observed (right) single signal .54

44. Voltage generation through (left) film bending and (right) compressive force from finger pulsing 55 


\section{INTRODUCTION}

\subsection{Nanogenerators}

With global energy consumption larger than ever before, the world has an urgent need for renewable and sustainable energy sources. Increasing power demands coupled with the emerging push for sustainable energy has led to a flux of new energy harvesting technologies within the last few decades. A novel type of energy harvesting device known as the nanogenerator is developing interest in the research world as a promising method to power small electronics from ambient energy sources. These nanogenerators utilize the specialized properties of nanomaterials to produce flexible, lightweight, and portable devices that have energy harvesting capabilities. This technology has been classified into three general categories based on their energy harvesting mechanisms: piezoelectricity, triboelectricity, and pyroelectricity.

The first type, the piezoelectric nanogenerator (PNG) is a device that can convert mechanical energy from tapping, bending, or vibrating into electrical energy through the flow of charges that neutralize a pressure induced polarization differential. The second type, the triboelectric nanogenerator also converts mechanical energy into electrical energy through a contact charging effect from the transfer of charges between surfaces of unlike materials. The final class of nanogenerators is the pyroelectric nanogenerator, which produces power by converting thermal energy into mechanical energy from the diffusion of charge carriers through a temperature gradient. ${ }^{7}$

Piezoelectric nanogenerators have developed into a flourishing area of research due their opportunities for facile processing resulting in the production of thin devices with high flexibility. The first proposed development of a nanogenerator was a PNG suggested by Professor Zhong Lin Wang at Georgia Institute of Technology. Wang's group observed the generation of electrical energy from an array of highly aligned zinc oxide nanowires when they were mechanically strained. ${ }^{8}$ Further tests from the group showed that zinc oxide nanowire devices could be used to drive electronic components such as LEDs and small liquid crystal displays when tapped, bended, or stretched. ${ }^{7}$ Since the group's establishment of nanogenerator technology in 2006, various other groups have investigated various combinations of nanomaterials that could directly generate 
piezoelectric energy ${ }^{9-11}$ or be incorporated into a piezoelectric material to enhance its energy harvesting efficiency ${ }^{12-14}$.

\subsection{Piezoelectric Effect}

To optimize the power conversion efficiency of a PNG, it is important to have a strong background understanding of its working mechanism, the piezoelectric effect. This effect was first discovered on August 2, 1880 by two brothers, Pierre and Jacques Curie, who were interested in studying the character of pyroelectric crystalline materials that would produce an electric potential from a change in temperature. The Curie brothers believed that this effect was the result of a material's asymmetric crystal structure, and that these structures might be able to generate electric potential from other direct stimuli such as mechanical stress. Using a simple setup of tinfoil, wire, and a jeweler's saw, the brothers tested various materials such as quartz, topaz, and Rochelle salt, and found a charge gradient would indeed form when applying mechanical strain. ${ }^{15,16}$

This was the first discovery of direct piezoelectric effect, and not long after, mathematician Gabriel Lippman found the reverse piezoelectric effect when he demonstrated that a piezoelectric material will change its structure when it is exposed to an external electric field. ${ }^{17}$ After these discoveries, interest in piezoelectric materials waned until the early $20^{\text {th }}$ century, when piezoelectric ultrasonic transducers were designed with quartz crystals for submarine sonar equipment. Shortly after, the technology was employed in various systems such as audio speakers and microphones, quartz watches, sensors, and MEMS actuators. ${ }^{18,19,20}$

The general definition of a piezoelectric material is a crystalline material with aligned noncentrosymmetric unit cells resulting in a unidirectional electric charge polarization throughout the crystal structure. These materials, also known as oriented-dipole electrets, exhibit a change in charge polarization density when strained as the spacing between charged atoms within the crystal lattice is compressed or extended. ${ }^{21}$

For example, lead zirconate titanate (PZT, chemical structure $\left.\mathrm{Pb}\left(\mathrm{Zr}_{x} \mathrm{Ti}_{1-\mathrm{x}}\right) \mathrm{O}_{3}\right)$ is a widely used piezoelectric ceramic with a perovskite structure (Figure 1). At room temperature, the unit cell of PZT is tetragonal, and the central $\mathrm{Ti}^{4+}$ or $\mathrm{Zr}^{4+}$ are displaced out of plane with surrounding $\mathrm{O}^{2-}$ ions, resulting in polarization. PZT is also defined as a ferroelectric material, a subclass of 
piezoelectric materials that have a spontaneous electric polarization that can be reversed through the application of an external electric field. At a molecular scale, polarization reversal occurs through the reorientation of the central $\mathrm{Ti}^{4+} / \mathrm{Zr}^{4+}$ from its equilibrium position above the central plane of the unit cell to a position of equal energy below the plane, leading to a $180^{\circ}$ reversal in the direction of spontaneous polarization. Above the Curie temperature $\left(T_{\text {curie }}\right)$ the material transitions from the ferroelectric phase to the paraelectric phase: PZT unit cell is face-centered-cubic, the central $\mathrm{Ti}^{4+} / \mathrm{Zr}^{4+}$ are at the body center in plane with surrounding $\mathrm{O}^{2-}$ ions, and the overall structure is nonpolar. ${ }^{22}$

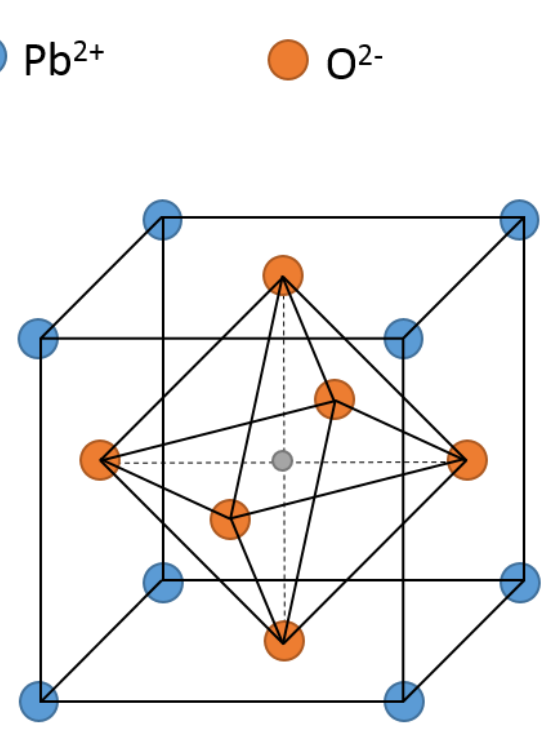

$\mathrm{T}>\mathrm{T}_{\text {curie }}$
$\mathrm{Ti}^{4+}, \mathrm{Zr}^{4+}$

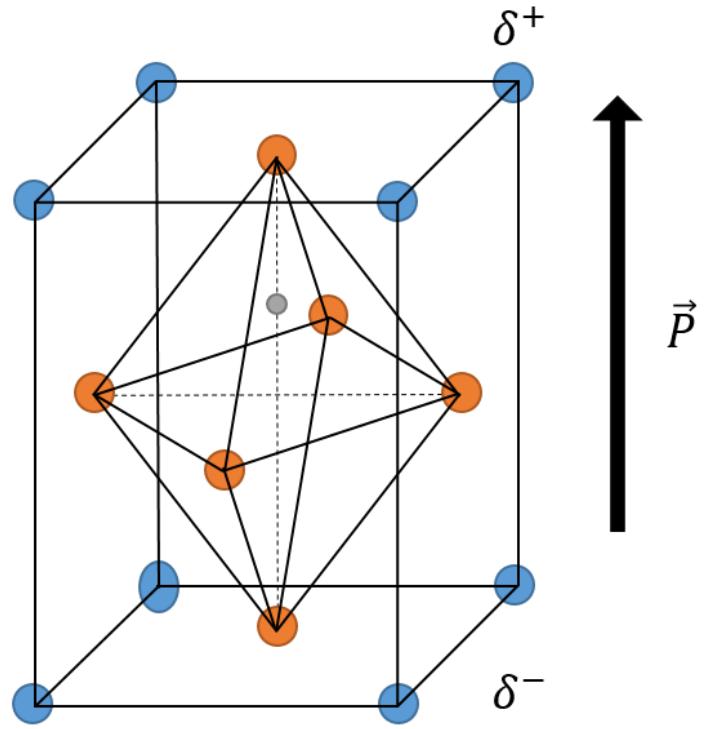

$\mathrm{T}<\mathrm{T}_{\text {curie }}$

Figure 1. Noncentrosymmetric perovskite unit cell of lead zirconate titanate with charge polarization across the structure below $\mathrm{T}_{\text {curie. }}$

Ceramics such as PZT possess domains in which the polarization of neighboring units align in the same direction, but between these domains the direction of the polar field vector may vary. Domain walls separate these regions, and the directions of polarization between neighboring domains are either $90^{\circ}$ or $180^{\circ}$ from one another. As a result, the net polarization across the material is essentially zero, as randomly oriented domains counteract each other's polarization. Unidirectional orientation of polar field vectors is established within a ferroelectric material through 
the application of an externally applied DC electric field below $\mathrm{T}_{\text {curie. }}$. From the application of the external field, the direction of polarization in the crystallites will align with the direction of the poling field. After the external field is removed, the resulting material is left with a remnant polarization $\left(\mathrm{Pr}_{\mathrm{r}}\right)$ perpendicular to the poling axis. ${ }^{21}$

(a)

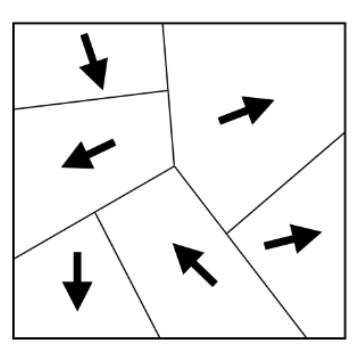

(b)

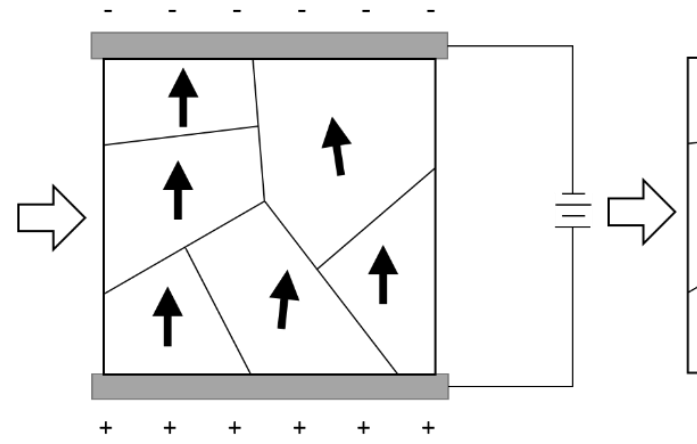

(c)

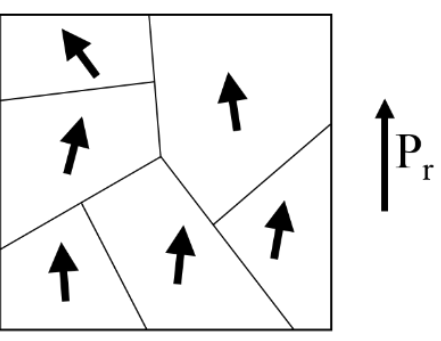

Figure 2. (a) Unpoled piezoelectric with randomly oriented ferroelectric domains, (b) during poling, an external voltage is applied to the material to align ferroelectric domains, (c) after poling, domains are oriented in similar directions, resulting in a remnant polarization $\left(\mathrm{P}_{\mathrm{r}}\right)$ in the direction of the applied field.

The characteristics of ferroelectrics are better understood by visualizing their polarization behavior using a ferroelectric (P-E) hysteresis loop (Figure 3). This test measures the polarization (P) of a ferroelectric material as a function of an applied alternating electric field (E). Electrodes across the surfaces of the ferroelectric sample $\left(C_{x}\right)$ are connected in series with a linear capacitor $\left(\mathrm{C}_{0}\right)$, so that the measured voltage across $\mathrm{C}_{0}$ is proportional to the polarization of the ferroelectric sample. As a large electric field is applied across the ferroelectric material, domains that have polarization oriented opposite to the direction of the applied field undergo switching into the direction of the applied field, essentially applying the poling treatment previously mentioned (curve $\mathrm{OA}$ in Figure 3). The extrapolation of the linear tangential segment of the $\mathrm{BC}$ section in Figure 3 back to the vertical axis (point $E$ ) represents the spontaneous polarization $\left(P_{s}\right)$. As the applied field is increased, a maximum polarization is reached as all domains are switched in the direction of the field. When the field is reduced back to zero, the polarization of the ferroelectric sample decreases 
to a non-zero $\operatorname{Pr}($ point $\mathrm{D})$. The applied field required to decrease the polarization to zero is the coercive field strength $\left(E_{c}\right)$. As the field strength increases in the opposite direction, the polarization of the ferroelectric domains reverse so that the spontaneous polarization is reversed. The P-E loop is completed by reversing the applied field direction once more. ${ }^{1}$

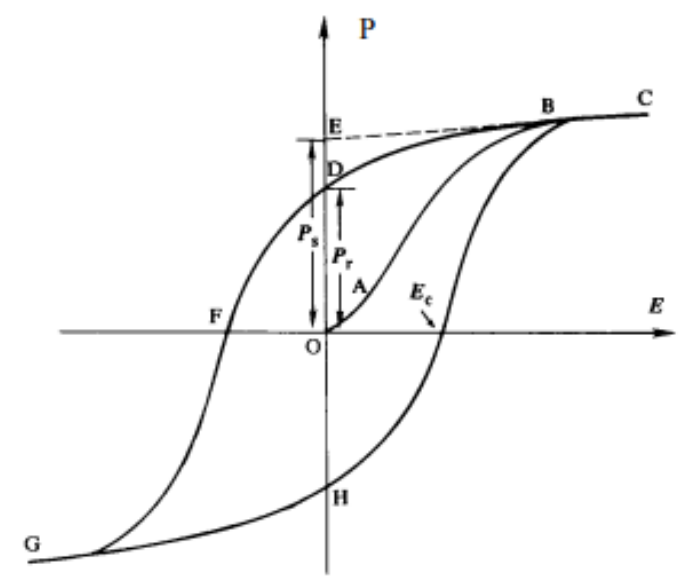

Figure 3. Typical ferroelectric (P-E) hysteresis loop. ${ }^{1}$

As previously mentioned, the compression or extension of the non-centrosymmetric unit cell of a piezoelectric material results in the change in charge polarization across it. When this compression-induced change in polarization occurs throughout a piezoelectric material with unidirectional orientation, the average polarization across the material changes, resulting in a change of charge at the surfaces on either side of the device. The application of conductive electrode films to these surfaces and connection with external conductive wires creates a pathway for charges to travel on either side of the polarization field. Thus, during compression, charges can run from one electrode to the other to compensate for changes in material surface charges. These charges continue to travel between electrodes until a new equilibrium is reached, resulting in electron flow towards the positively charged electrode. When the material is decompressed, it returns to its original polarization state, and charges transfer to their original state resulting in an electron flow in the opposite direction. 

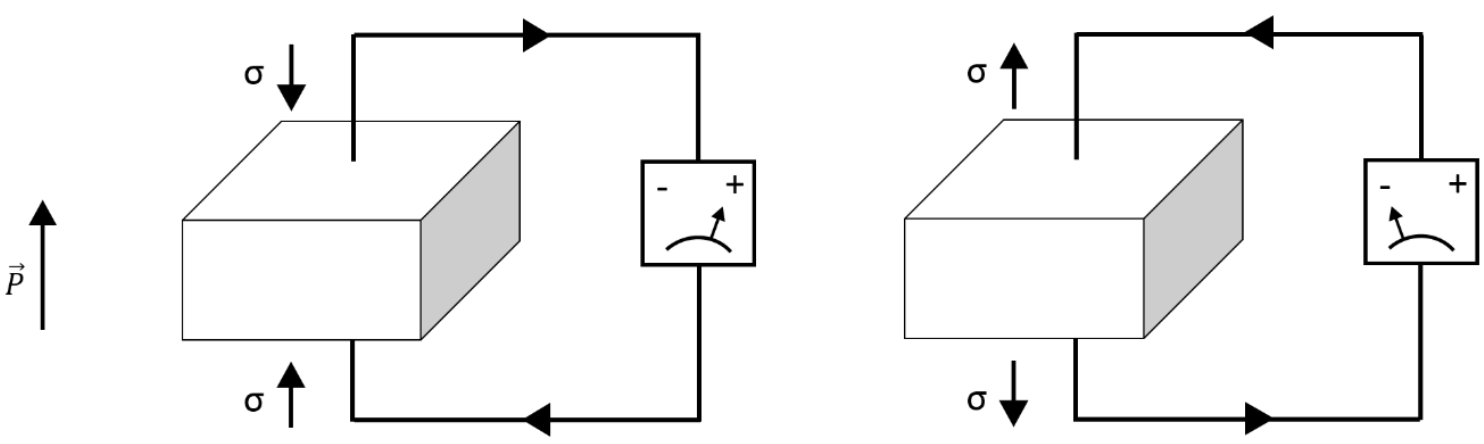

Figure 4. Generation of current across electrodes as a piezoelectric material is compressed and decompressed.

The piezoelectric effect is described as the generation of an electrical charge density as a result of an applied stress through the equation:

$$
D=\frac{Q}{A}=d \sigma
$$

In which the charge density $(D)$ is charge $(Q)$ per unit area $(A)$, which is equivalent to the applied stress $(\sigma)$ multiplied by the piezoelectric charge constant $(d) .{ }^{23}$

Piezoelectric materials can also undergo the reverse piezoelectric effect, described by the following equation:

$$
\varepsilon=d E
$$

Here, the resulting strain $\varepsilon$ is generated from a field $E$, and these values are proportionally related with the piezoelectric charge constant. When considering the both the direct and inverse piezoelectric effect, the electrical charge density is described as:

$$
D=d \sigma+\epsilon^{\sigma} E
$$

In which $\epsilon^{\sigma}$ represents the film's dielectric permittivity under a zero or constant stress. ${ }^{24}$ The above equation is rewritten to better describe the behavior of a piezoelectric transducer:

$$
E=g \sigma+\beta^{\sigma} D
$$

In which $g$ represents the piezoelectric voltage constant and $\beta^{\sigma}$ represents the impermitivity of the film under a zero or constant stress. These equations are generally expressed in vector and tensor notation, but for the purpose of this introduction they have been generalized. 
${ }^{25}$ These relationships only hold true when piezoelectric materials are linear, at low electric fields (reverse piezoelectric effect) and low mechanical stress (direct piezoelectric effect). Above these conditions, transducers display a non-linear hysteresis-like behavior.

Equations 3 and 4 are generalized to describe the piezoelectric charge and voltage constants expressed as a function of developed charge density or electric field as a function of imparted stress:

$$
\begin{aligned}
& d_{i j}=\frac{D}{\sigma} \\
& g_{i j}=\frac{E}{\sigma}
\end{aligned}
$$

A high piezoelectric charge constant is desirable for materials that undergo high frequency vibration such as ultrasonic transducers, whereas a high piezoelectric voltage constant, $g$, is desirable for materials that generate large voltages in response to mechanical stress. ${ }^{23}$ When $d$ and $g$ constants are reported in literature, they are often reported in with "i" and "j" indices, which indicate the direction of induced polarization or voltage in relation to the applied stress, respectively. These indices indicate directional vectors 1 to 3 signifying the $x, y$, and $z$ axis directions, respectively, and 4 to 6 representing shear around the respective axis (Figure 5).

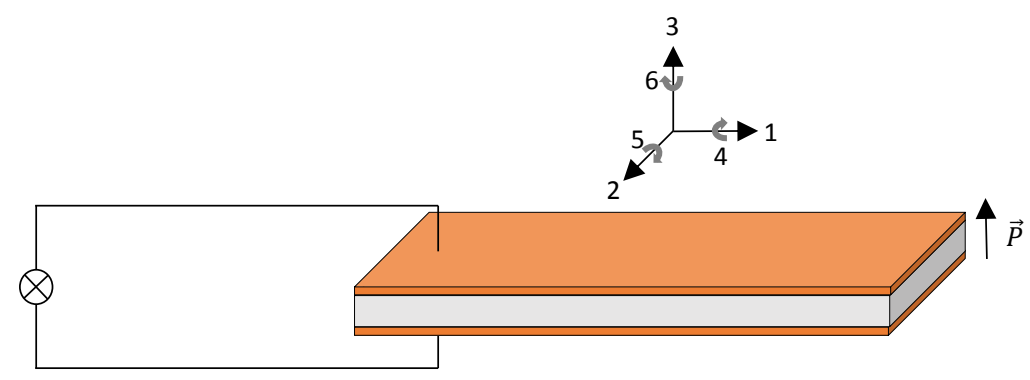

Figure 5. General diagram of piezoelectric transducer with directional axis 1, 2, and 3 and shear axis 4,5 , and 6 .

For example, the $d_{33}$ coefficient is the piezoelectric charge coefficient that corresponds to the electrical charge density normal to the direction of the film per unit of stress imparted normal to 
the film's direction. A more general way to describe this term is the amount of charge density generated across the electrodes of a piezoelectric transducer such as the one in Figure 5 per unit stress applied normal to the film from a normal compressive force such as tapping.

\subsection{Piezoelectric Polymers}

Recently, there has been a large push to use materials other than inorganics in electronics technology due to their poor sustainability, brittleness, toxicity, and complicated processing. Functional polymers are a popular class of materials that are being used to replace inorganics in electronics technology due to their facile solution-based batch synthesis and processing, high flexibility, and low toxicity. Functional polymers have been integrated into many electronics technologies including field effect transistors (FETs), light emitting diodes (LEDs), photovoltaics, and battery technologies. A recent report from IDTechEx, an independent market research organization recognized as a leading expert on emerging technologies suggests that market for printable, flexible organic electronics is forecasted to grow from $\$ 31.7$ billion in 2018 to $\$ 77.3$ billion in 2029. ${ }^{26} \mathrm{~A}$ portion of this market growth has been forecasted to occur within piezoelectric polymer nanogenerator technologies.

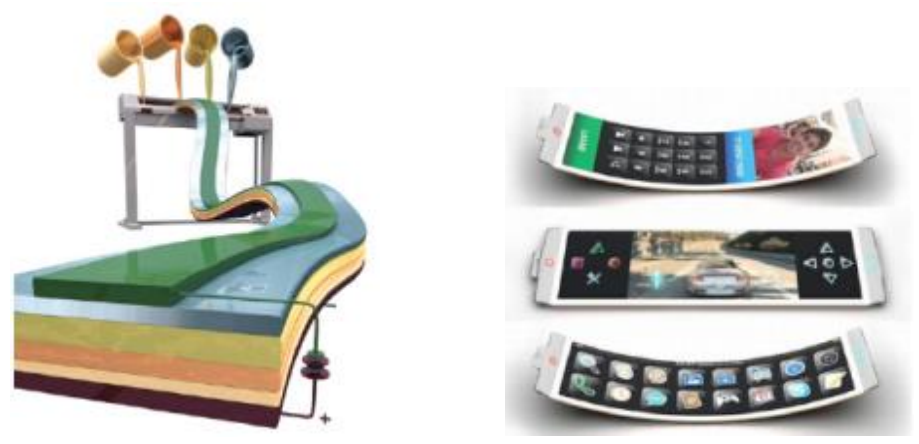

Figure 6. Facile solution-based processing of polymer electric components gives rise to opportunities for a new class of flexible and sustainable polymer electronics. ${ }^{27}$

The piezoelectric effect was originally believed to exist only in inorganic materials, but in 1925, physicist Mototaro Eguchi discovered that when a molten mixture of carnauba wax and resin was heated to $130^{\circ} \mathrm{C}$ and exposed to a $1.5 \mathrm{MV} / \mathrm{m}$ electric field, injected charges from contacted electrodes would form a charge on the surface of the mixture resulting in charge polarization across 
the material. Dielectric materials like Eguchi's carnauba wax that hold induced charges are regarded now as an important class of piezoelectric materials known as real-charge electrets. ${ }^{28,29}$ It was discovered later that these real-charge electrets trap and store charges either on the material's surface or within the material, which results in a polarization analogous to that of an oriented-dipole piezoelectric. In reality, most piezoelectric polymers have been suggested to display both real-charge and oriented-dipole piezoelectric behavior.

In 1969, Dr. Heiji Kawai of the Kobayashi Institute of Physical research in Tokyo discovered that a large piezoelectric voltage signal is generated from structurally regular poly(vinylidene fluoride) (PVDF) when properly processed. ${ }^{28,30}$ Kawai noticed that the repeat unit of this polymer, which has chemical structure $\left(\mathrm{CH}_{2}-\mathrm{CF}_{2}\right)$, has an exceptionally large dipole moment of $2.1 \mathrm{D}^{3}$ when oriented in the all-trans conformation. When this polymer forms into an all-trans crystalline state, it forms a non-centrosymmetric unit cell with oriented-dipole piezoelectric behavior similar to that of inorganic piezoelectrics. In this case, to make a working piezoelectric device from this polymer, the all-trans conformation of the polymer must be formed and regularly oriented normal to the direction of the film's plane.

To achieve this desired orientation within the polymer system, the proper crystal domains must be formed through certain processing methods. In a good solution, polymers exist in an amorphous state in which polymer chains are in a random-coil orientation (Figure 7a). With flexible, linear chain polymers, aligned crystalline domains are formed either through orientation by mechanical stretching or through self-organized regular alignment and folding of polymer chains as the solvent is evaporated. The latter crystal formation mechanism occurs through intermolecular forces between chain segments and results in sheet-like crystals called lamellae. As polymers rarely form $100 \%$ crystalline films when formed from these conditions, crystal lamellae are normally dispersed among amorphous polymer regions in what is known as a semi-crystalline matrix. 

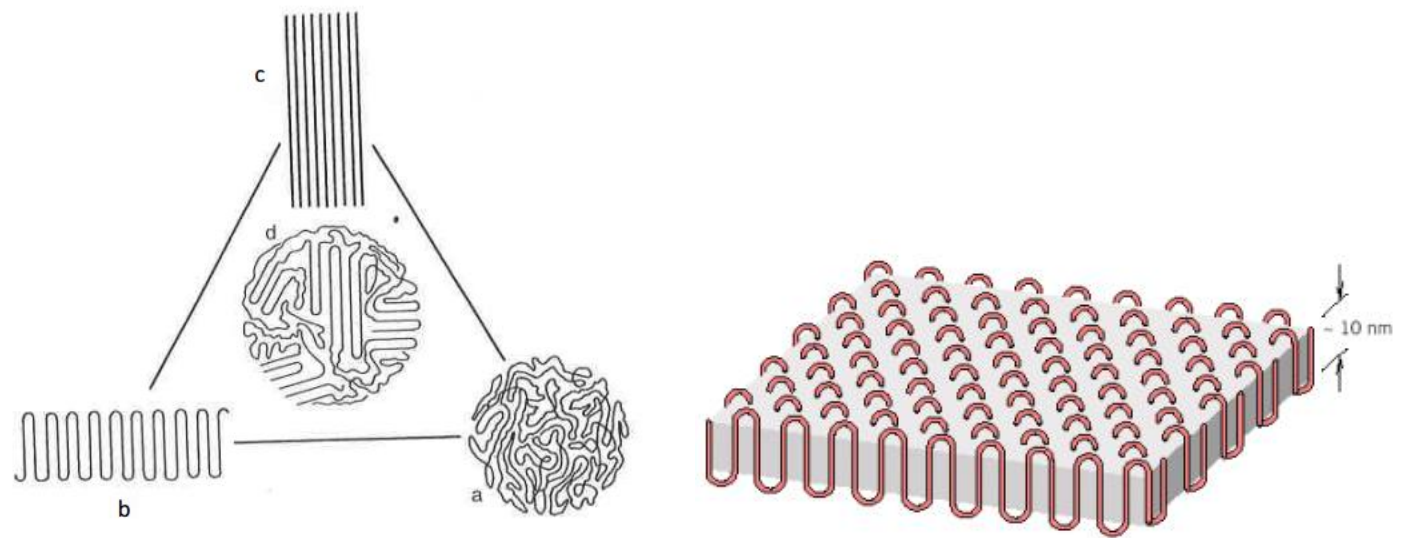

Figure 7. (Left) Different conformations in linear polymers (a) amorphous conformation (b) lamellar sheets (c) extended chain crystals (d) semi-crystalline matrix. (Right) Illustration of polymer crystal lamellae. ${ }^{31}$

When PVDF is crystallized under quiescent conditions (either from dilute solution or the melt), chains orient themselves in order to minimize steric and electrostatic repulsion between adjacent fluorine groups, and will assume the lowest energy torsional bonding arrangements of either 180 degrees (trans, $\mathrm{t}$ ) or \pm 60 degrees (gauche ${ }^{ \pm}, \mathrm{g}^{ \pm}$) between substituents when forming lamellar structures. As a result of multiple low energy rotational states, PVDF is a polymorph, and

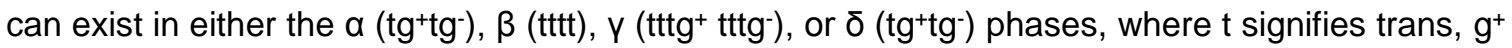
signifies clockwise gauche, and g- signifies counterclockwise gauche bond rotation. Although both the $\alpha$ and $\delta$ crystal chain orientations have identical rotational order leading to a formed dipole moment, stacking of $\alpha$ chains form antiparallel crystal packing leading to a nonpolar internally compensated crystal domain (Figure 8). To form $\delta$-phase crystals, the $\alpha$ phase is introduced to an external electric field, and transverse chains rotate $180^{\circ}$ along its torsional axis leading to dipole alignment within the crystal lamellae (Figure 8). In comparison, the all-trans $\beta$ phase crystal forms overall unidirectional polarization within the crystal lamellae without the need for an applied field. The $y$ phase crystal also forms polarization throughout its domain, although dipole moment per 
repeat unit is significantly smaller than that of a $\beta$ phase crystal. ${ }^{2}$ Thus, to develop a piezoelectric device from PVDF, it is important to maximize the formation of $\beta$-phase crystal domains.
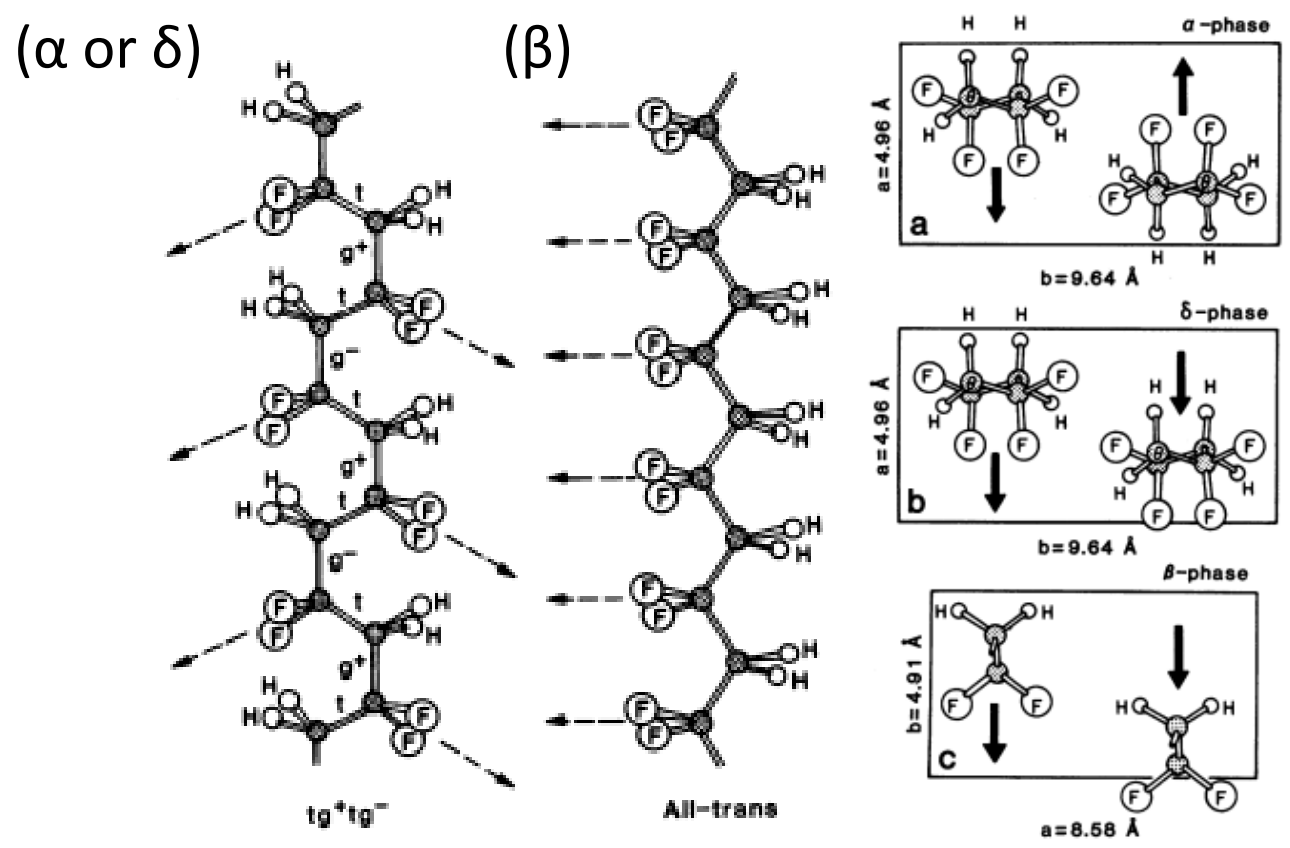

Figure 8. (Left) Depiction of crystalline chain conformations of PVDF $\alpha / \delta$ and $\beta$ phases. (Right) Unit cells of the (a) $\alpha$-phase (b) $\delta$-phase (c) $\beta$-phase of PVDF projected parallel to chain axes. ${ }^{2}$

Although most linear polymers form an all-trans conformation when crystallized from quiescent conditions, steric repulsion between fluorine groups causes PVDF's lowest energy crystal conformation to be the nonpolar a-phase, rendering the film nonfunctional as a piezoelectric device. As well, even if crystal domains within the semi-crystalline polymer matrix were polarized, the crystalline domains would be randomly oriented, and unidirectional orientation of polarized domains is needed for an effective piezoelectric device.

Kawai developed a processing method to achieve unidirectionally oriented $\beta$-phase crystal domains of PVDF that is utilized to this day. In essence, this method involves mechanical extension and then electrical poling of a film formed from quiescent conditions (Figure 9). Mechanical drawing at temperatures below $90^{\circ} \mathrm{C}$ is responsible for reorienting the spherulitic $\alpha$ phase crystals to the chain's fully extended all-trans $(\beta)$ conformation in the direction of drawing. At this point, polarized crystal domains are formed, but their dipole vectors must be oriented normal to the film, which is 
achieved through external poling normal to the film from a high voltage source of at least $0.4 \mathrm{MV}$ $\mathrm{cm}^{-1} \cdot{ }^{2}$
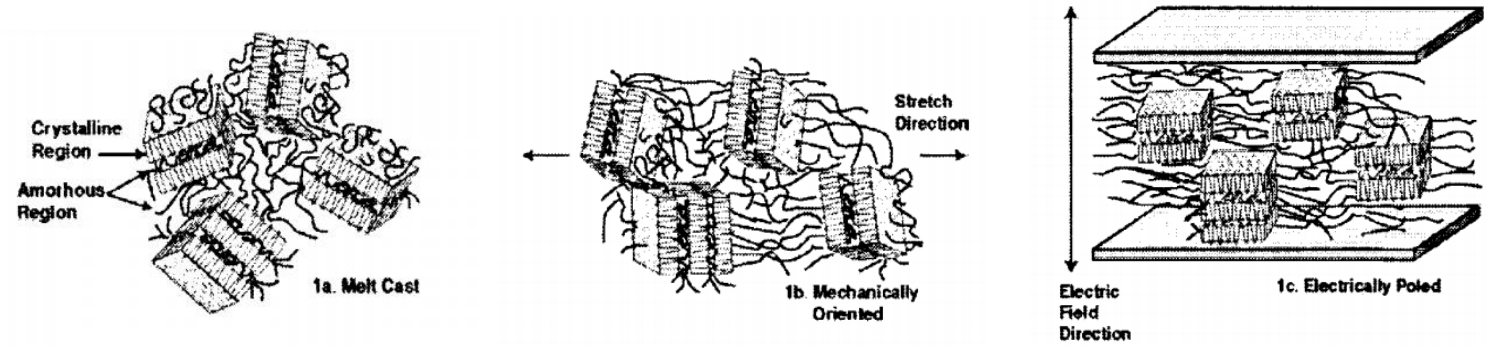

Figure 9. Schematic illustration showing (left to right) morphology of film after melt casting ( $\alpha$ phase), mechanical stretching to reorient chains to the extended $\beta$-phase, and application of external electric field to align polarization domains of crystals. ${ }^{3}$

This stretch and pole processing method, while effective, requires specialized processing equipment and the application of a strong electric field resulting in the use of a large amount of additional energy and added processing equipment. To combat the need for PVDF stretching, researchers have developed copolymers of PVDF that exhibit a strong piezoelectric effect with high crystallinity and the formation of the polar $\beta$-phase from the melt.

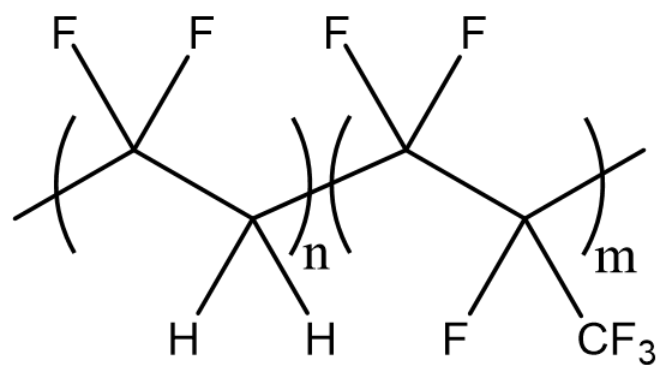

$\mathrm{P}($ VDF-HFP $)$

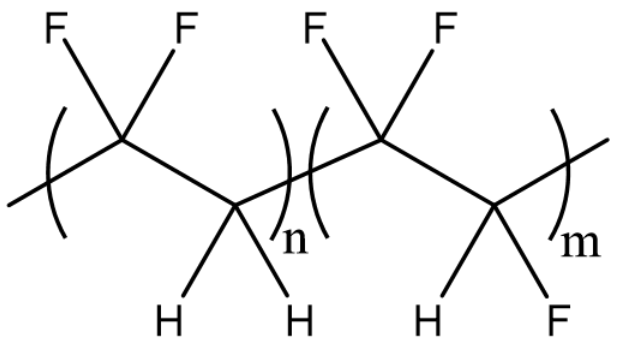

$\mathrm{P}(\mathrm{VDF}-\mathrm{TrFE})$

Figure 10. Structure of PVDF copolymers (left) poly(vinylidene fluoride-co-hexafluoropropylene) and (right) poly(vinylidene fluoride-co-trifluoroethylene).

Of these copolymers, poly(vinylidene fluoride-co-trifluoroethylene), abbreviated P(VDFTrFE) (Figure 11) is of key interest in this study. P(VDF-TrFE) has been established as the standard 
piezoelectric polymer in research and industry due to its excellent ferroelectric properties and ability to form electroactive domains from the melt. At $25 \mathrm{~mol} \%$ TrFE, the copolymer has a $\mathrm{d}_{33}=-24 \mathrm{pC} / \mathrm{N}$ (at $110 \mathrm{~Hz}$ ), a $\mathrm{T}_{\text {curie }}$ of $116^{\circ} \mathrm{C}$, and a melting point of $145^{\circ} \mathrm{C}$. The higher proportion of bulky fluorine groups in $\mathrm{P}(\mathrm{VDF}-\mathrm{TrFE})$ copolymers with 60 to 80 mole \% VDF prevents the formation of the $\mathrm{tg}^{+} \mathrm{tg}^{-}$ $\alpha$ phase through steric repulsion, leaving the all-trans $\beta$-phase conformation as the preferred lowest energy crystal structure. As a result, the stretch processing step is forgone as this copolymer produces high levels of $\beta$-phase crystal structure directly through crystallization from quiescent conditions. This copolymer also exhibits a Curie temperature that can be observed experimentally because it is below its melting temperature, unlike the $\mathrm{T}_{\text {curie }}$ of PVDF and P(VDF-HFP). ${ }^{2}$

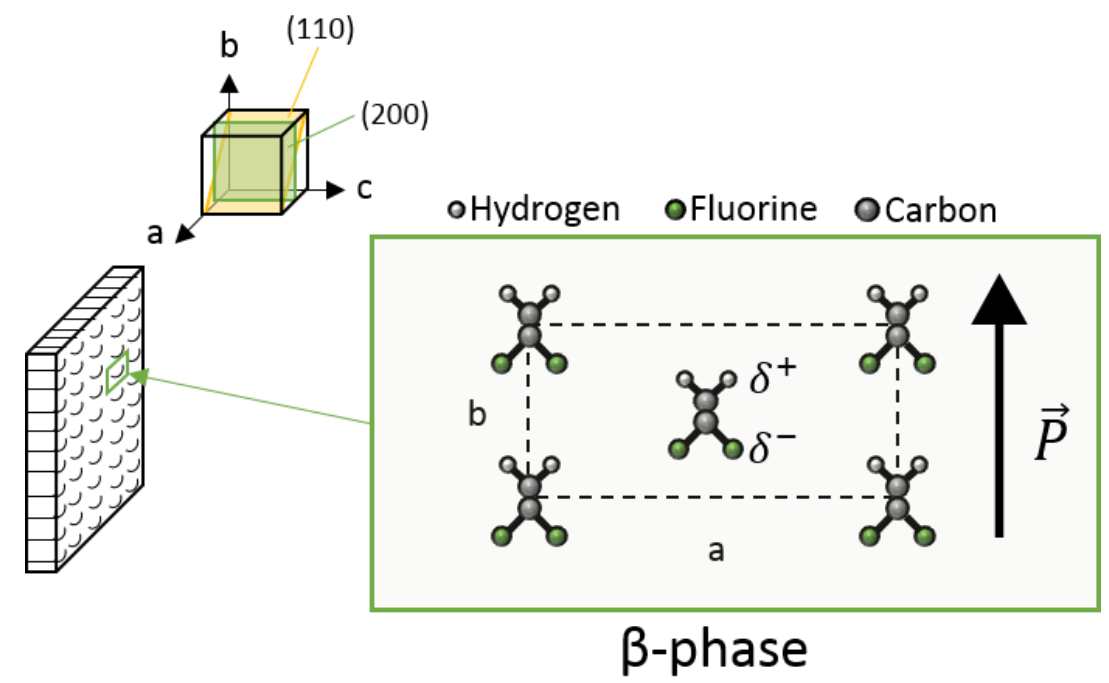

Figure 11. Orientation of $\beta$-phase within the PVDF lamellar crystal throughout the $a, b$, and c axis. The (110) and (200) crystallographic planes shown in the upper left corner are commonly associated with the $\beta$-phase of (PVDF-TrFE) and are visible around $19.9^{\circ}$ using X-ray diffractometry. ${ }^{4}$

The copolymer poly(vinylidene fluoride-co-hexafluoropropylene), abbreviated P(VDF-HFP) is a polymer often studied for polymer electrolytes, but it has been shown to have strong piezoelectric properties and good charge-trapping properties leading to additional oriented dipole stabilization. ${ }^{32}$ PNGs made from this cost-effective copolymer were prepared and their energy harvesting ability was compared to PNGs prepared with $\mathrm{P}(\mathrm{VDF}-\mathrm{TrFE})$. 


\subsection{Nanomaterials}

Recent interest in nanomaterials has risen within the last few decades due to the unique properties that they possess. They have been involved in nearly every facet of the research world, including use for reinforcing materials, additives to introduce new functionalities, and nanostructures for electronics. These materials, also called nanoparticles (NPs) are classified as particles that are between 1 and $100 \mathrm{~nm}$ in at least one dimensional axis. As a result of their small size, they have a very large surface area-to-volume ratio, resulting in large amounts of interfacial contact with the surrounding environment within a small volume of particles. For example, a solid cube with $1 \mathrm{~cm}$ sides will have an overall volume $1 \mathrm{~cm}^{3}$ and $6 \mathrm{~cm}^{2}$ surface area, while a powder of $1 \mathrm{~nm}$ cubes with a $1 \mathrm{~cm}^{3}$ volume has a total surface area of $6 \times 10^{7} \mathrm{~cm}^{2}$ (Figure 12). The surface-tovolume ratio of the powder is seven orders of magnitude, or ten million times larger than the solid cube.

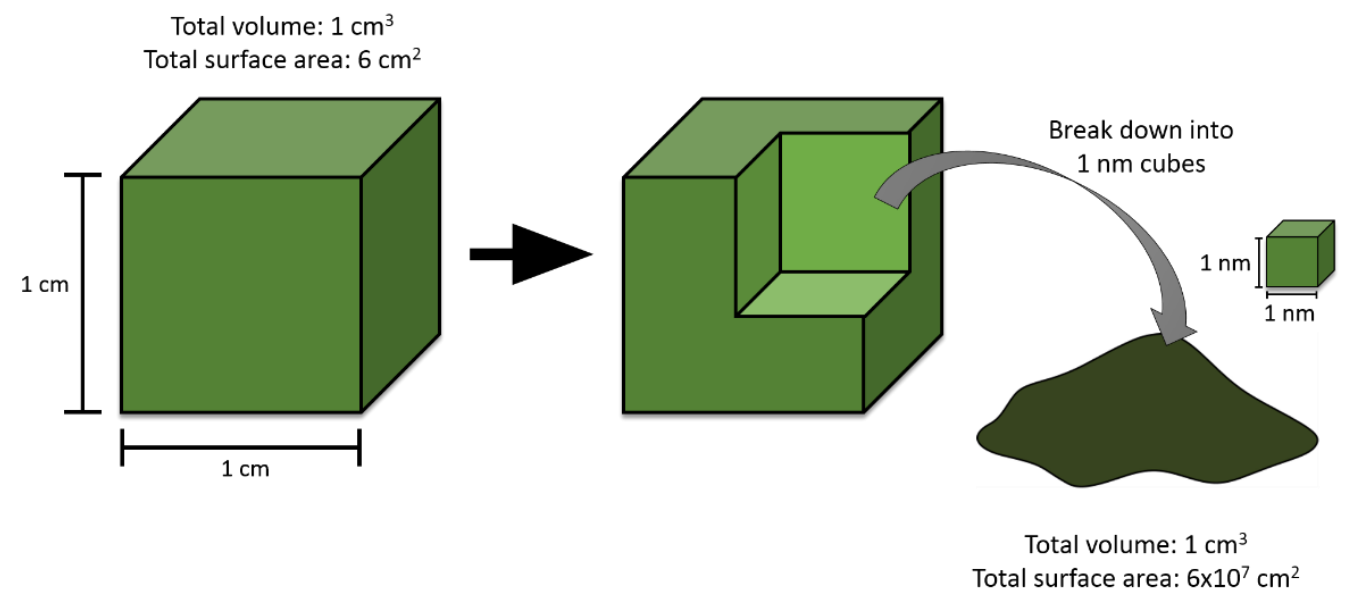

Figure 12. As the particle size within a constant volume decreases, the surface area to volume ratio increases significantly.

Another advantageous property of nanoparticles is that they are made from a multitude of different materials, including metals and metal oxides, polymers, and carbon nanomaterials. Intrinsic properties of nanoparticles are based on their composition, size, shape, crystallinity, and surface chemistry, all of which can be fine-tuned by synthesis and post-processing to obtain desired functionality. 
Typically, nanoparticle synthesis results in growth of surfaces bounded by the lowest energy facets, often leading to isotropic geometries. Methods of selectively controlling the growth of nanoparticles have been developed and generally consist of kinetic control, surface passivation onto a particular surface facet, and physical templating from a seeded substrate surface. Of those methods, surface passivation by use of a capping agent resulting in the controlled growth of exposed facets is utilized in this study due to the ease and versatility of the technique. ${ }^{33,34}$

Proper design of a nanoparticle shape is key to exploit their physical, optical, electrical, and catalytic properties. Nanomaterials are typically categorized as either 0-dimensional, 1dimensional, or 2-dimensional structures. These categories of classification are based on the length scale of the particle in each spatial dimension. For example, 0-dimensional nanoparticles are between 1 and $100 \mathrm{~nm}$ in all three dimensions, and thus usually have symmetrical spherical or cubic structures. The small size of these nanoparticles results in interesting quantum effects as they approach the quantum scale. As such, 0-dimensional semi-conducting nanoparticles begin to approach the size of an electron's De Broglie wavelength, which results in the quantum confinement of internal electrons, allow for the bandgap tunability of the nanoparticles. ${ }^{35}$

On the other hand, 1-dimensional nanoparticles have one dimension outside of the nanoscale that lead to high aspect ratio needle-like nanomaterials such as nanowires (NW), nanotubes (NT), and nanorods (NR). The morphology of these nanoparticles leads to interesting anisotropic properties either within a single particle or throughout a mixture of unidirectionally oriented particles.

Nanoparticles with two dimensions outside of the nanoscale, predictably labeled 2dimensional nanoparticles, make planar nanomaterials such as sheets and platelets. When nanosheets are layered parallel to the plane of a substrate, they can impose a tortuous path that lead to strong barrier properties for chemicals. As well, coatings with added sheet-like fillers show a sharp contrast in reflected light depending on the viewing angle as a result of anisotropic light attenuation at varying angles of incident light. This phenomenon, known as "flop", results in an observed darkening at larger viewing angles as incident light at these angles gets attenuated between the sheets. 
Mixtures of polymer matrix and nanoparticle fillers, known as polymer nanocomposites (PNCs), possess the properties of both the polymer and added nanofiller. These formulations can be customized for desired properties by proper selection of polymer and nanoparticle material and morphology. The first study of PNCs, which took place in the 1940s, sought to enhance the structural properties of rubber tires, and afterwards interest in the technology died down until the 1990's, when a study from Toyota Central Research showed that exfoliated montmorillonite clay could increase the modulus of nylon- 6 by a factor of around 3 as well as increase the heat deflection temperature by $80 \mathrm{~K} .{ }^{36,37}$ The key to enhanced properties of PNCs is the high surface volume of nanofiller in contact with the polymer phase. For example, a system with a particle volume fraction of 0.3 , when the particle diameter is $300 \mathrm{~nm}$, the interfacial volume fraction is 0.03 , whereas when particle diameter decreases to $50 \mathrm{~nm}$, the interfacial volume fraction rises to 0.22 . As such, NPs are used as effective fillers to optimize interfacial interactions. ${ }^{38}$

One such interfacial interaction is interfacial crystallization which is induced by secondary nucleation on a particle or molecule not made up of the crystallizing polymer. Interest has developed for the use of NPs in nanocomposites as nucleation surfaces to induce polymer crystallization in the hopes of maximizing the crystallinity of polymer matrix. This phenomenon is described by the Lauritzen-Hoffman theory of secondary nucleation, which is considered the standard model to understand the formation of polymer lamellae through interaction with a substrate surface.

Based on the Lauritzen-Hoffman theory, the chain nucleation step involves the initial stem deposition through the adsorption and flattening of a section of the chain onto a substrate surface, forming the activated state. As entropy decreases from this step, the primary stem deposition is the highest energy, and thus slowest stage in secondary nucleation. Therefore, favorable interactions between the nanoparticle surface and the initial section of polymer is crucial to overcome this energy barrier. Crystal formation continues with chain folding of the remaining attached polymer and the elongation and formation of a secondary activated state parallel to the primary stem. These subsequent steps continue until the lamellar structure forms along the lateral surface formed by the first stem (Figure 13). 5 
(a)

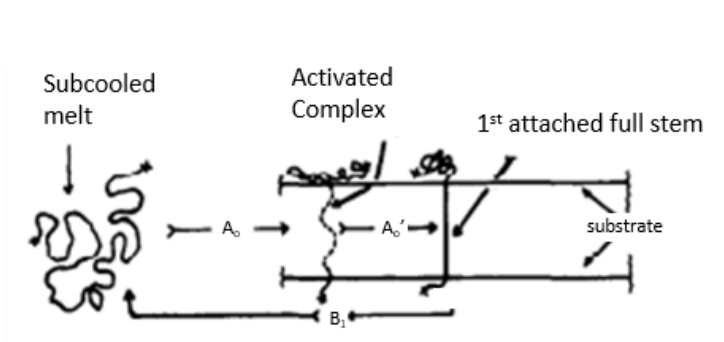

(b)

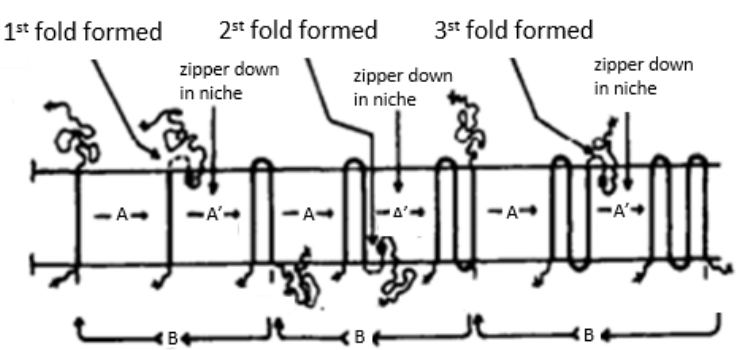

Figure 13. Illustration of (a) Initial step deposition on substrate surface and (b) subsequent stem and fold formation resulting in development of crystal lamella. ${ }^{5}$

Various studies have demonstrated that the high surface area-to-volume ratio of NPs make them excellent nucleation agents for inducing polymer crystallization of PVDF and its copolymers through interfacial interactions. These studies have shown that the fraction of $\beta$-phase of PVDF drastically increases with addition of various nanofillers such as ferrite NPs (Figure 14), 6 piezoelectric $\mathrm{BaTiO}_{3},{ }^{39}$ and acid functionalized MWCNT ${ }^{40}$. Nanocomposites with the P(VDF-HFP) copolymer showed similar trends on the increase in $\beta$-phase from the addition of NPs such as $\mathrm{SiO}_{2}$ nanorods, ${ }^{41} \mathrm{Ni}$-doped $\mathrm{ZnO}$ NPs, ${ }^{42}$ and Pt NPs ${ }^{12}$. On the other hand, literature does not show a clear trend on NP influence on $\beta$-phase formation within $\mathrm{P}(\mathrm{VDF}-\mathrm{TrFE})$ nanocomposites. For example, it was reported that the amount of $\beta$-phase is not effected by the addition of organically modified layered silicates ${ }^{43}$ or gold nanowires ${ }^{44}$, even though they report enhanced piezoelectric properties from the nanocomposites due to other interfacial interactions. 

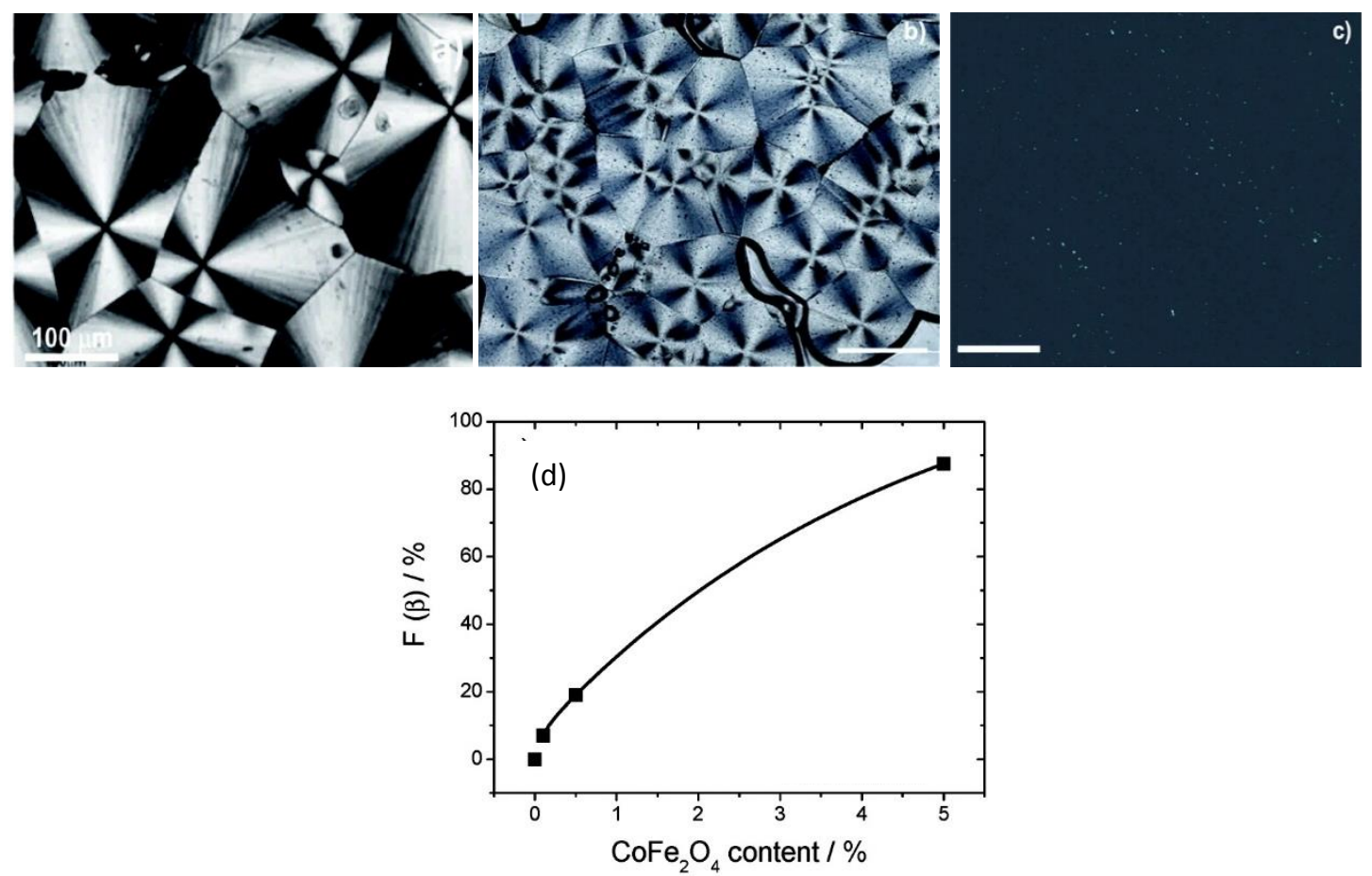

Figure 14. Polarized optical microscopy images of (a) PVDF, (b) $0.1 \% \mathrm{CoFe} 2 \mathrm{O} 4$, and (c) $5 \%$ CoFe2O4 PNCs (200 $\mu$ m scalebars in b and c). (d) Increase in $\beta$-phase in nanocomposites with increasing CoFe2O4 nanocomposite sample measured through FTIR. ${ }^{6}$

As interest in PNG technology increases, various studies are focusing on determining methods of increasing the piezoelectric performance of polymer PNGs through the addition of nanofillers. The scope of these studies covers a broad range of processing and characterization techniques, but they generally seek to determine methods of increasing the piezoelectric characteristics of the films through the addition of nanofiller to act as nucleating surfaces for polymer crystallization or as intrinsic piezoelectric materials that directly contribute to power harvesting. In this study, 1-dimensional nanomaterials are used as nanofillers in order to investigate the influence of their high aspect ratios (length/diameter, L/D) on the PNG performance. These special morphologies, formed through surface passivation to promote directional growth, ${ }^{38}$ result in extremely high surface area-to-volume ratios, and resulting particles have a directional dependency in their mechanical, electrical, and optical properties which can be taken advantage of for applications that require directional orientation. 
Zinc oxide nanowires (ZnO NW) are a highly utilized nanofiller in PNG studies due to their intrinsic piezoelectric properties and capability for unidirectional alignment. These materials have both piezoelectric and semiconducting properties (with a band gap of $3.37 \mathrm{eV}$ ) ${ }^{45}$ which make them versatile materials with demonstrated success for use in photocatalysis, sensing, UV detection, field effect transistors (FETs), and PNGs. ${ }^{46}$ The use of these materials in piezoelectric energy harvesters is of special interest due to their facile synthesis, highly customizable nanostructures, strong piezoelectric properties, and high biocompatibility and biosafety. ${ }^{8}$

Of the morphologies obtainable with $\mathrm{ZnO}$, high aspect ratio nanowires have been the focus of many studies due to the directionality of piezoelectric power generation in highly oriented arrays. $\mathrm{ZnO}$ can have a hexagonal wurtzite structure composed of tetrahedrally coordinated $\mathrm{O}^{2-}$ and $\mathrm{Zn}^{2+}$ ions stacked along the [0001] c-axis (Figure 15). Similar to tetragonal PZT, uneven charge distribution throughout the unit cell of wurtzite $\mathrm{ZnO}$ results in a polarization that can be stressed to generate energy.

As mentioned, the first group to introduce the concept of piezoelectric energy harvesting was the Wang group at Georgia Tech, who used highly aligned arrays of wurtzite $\mathrm{ZnO}$ NW grown along the [0001] direction. The group demonstrated that the deflection of a nanowire with a $\mathrm{Pt}$ coated conductive AFM tip generated a piezoelectric potential within the nanowire structure. The metal-semiconductor contact with the positively charged stretched side of wire formed a reversebiased Schottky diode. Contact with the compressed negatively charged side of the wire formed a positively biased Schottky diode, and an electric current would flow to neutralize the displaced ionic charges in the nanowire. ${ }^{8}$ This work is the initial study responsible for sparking the boom in research focused on energy harvesting technologies that have been developed to this day. 
(a)

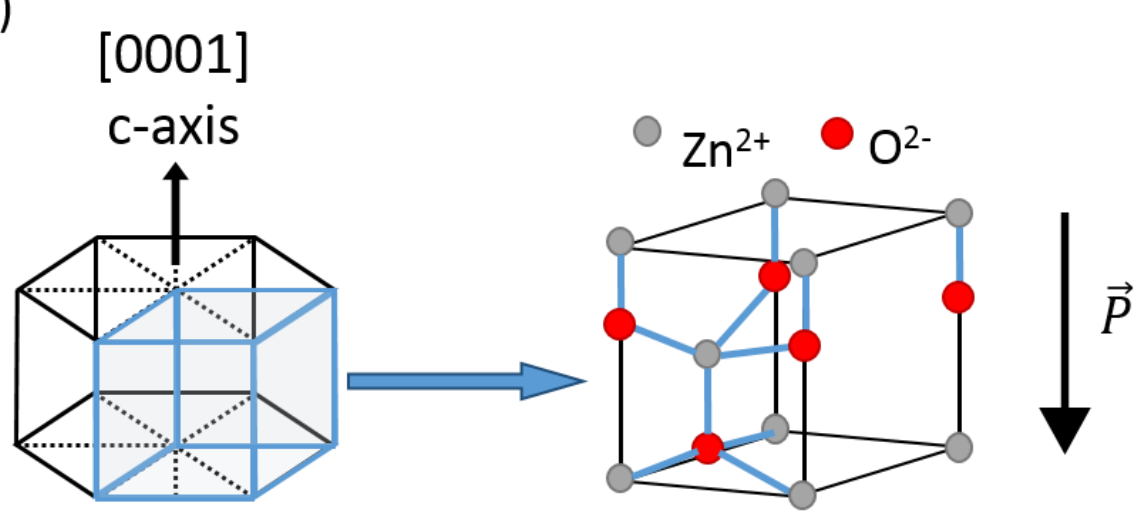

(b)

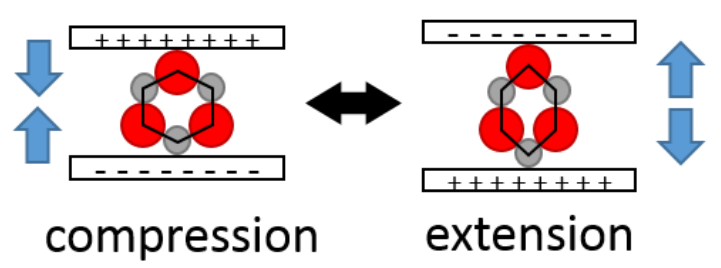

Figure 15. (a) Crystal structure of hexagonal wurtzite $\mathrm{ZnO}$ with a non-centrosymmetric unit cell (b) compression and extension of ions lead to changes in polarization which are stabilized by charge generation at electrode surfaces.

Another class of nanoparticles known as carbon nanomaterials have attracted attention due to their unique mechanical, thermal, electronic, optical, and chemical properties. This class of materials includes fullerenes (OD), carbon nanotubes (CNTs) (1D), and graphene (2D), all of which have different morphologies but share an identical carbon-lattice based structure, which consists of $\mathrm{sp}^{2}$ hybridized two-dimensional lattice of carbon in a honeycomb-like cyclohexane matrix. The m-bonding found between carbons throughout the lattice leads to widespread electron delocalization allowing electron transport over long lengths. A flat, two-dimensional plane of this lattice as described above is known as a graphene sheet, and a CNT is made when this sheet is rolled over on itself to create a cylindrical shape.

Carbon nanotubes are characterized as either single-walled (SWCNT) or multi-walled (MWCNT) based on the amount of graphene layers surrounding the core nanotube, where a SWCNT only has one core cylinder and a MWCNT has multiple layers stacked upon each other 
(Figure 16). Of the three carbon-based nanomaterials, carbon CNTs have been highly anticipated due to their attractive mechanical, electrical, thermal, and optical properties. Their high aspect ratio leads to superior charge transport along its pseudo-1 dimensional lattice without interruption making them more conductive than copper. ${ }^{47}$ These materials have been utilized in all branches of science and technology for uses such as drug delivery, energy storage, and even nano-sized circuitry. ${ }^{48-50}$

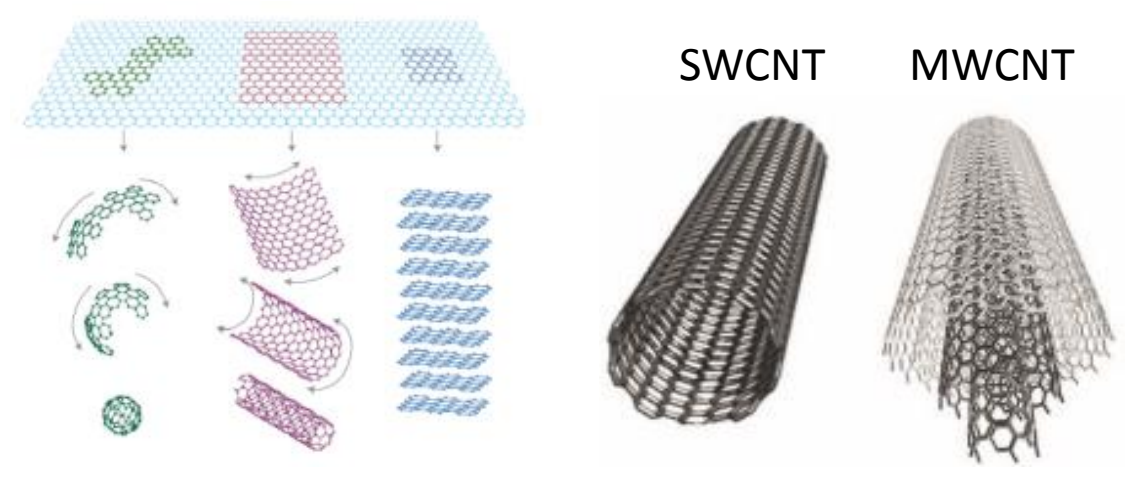

Figure 16. (a) Lattice structure of carbon nanomaterials and (b) Structure of single-walled (left) and multi-walled (right) carbon nanotube. ${ }^{51,52}$

Due to their distinct character, CNTs have been extensively used as nanofillers in piezoelectric PNCs to modify their energy harvesting capabilities. One study developed a highly flexible nanogenerator consisting of $12 \mathrm{wt} \%$ piezoelectric barium titanate $\left(\mathrm{BaTiO}_{3}\right)$ nanoparticles and either 1 wt \% SWCNT or MWCNT dispersed in a PDMS matrix. It was found that PDMS films with both $\mathrm{BaTiO}_{3}$ and either SW/MWCNT produced an output voltage $\sim 3 \mathrm{~V}$, compared to an output voltage of $\sim 0.15 \mathrm{~V}$ from PDMS films with just $\mathrm{BaTiO}_{3}$. The group theorized that the CNT network increases the dispersion of $\mathrm{BaTiO}_{3}$ nanoparticles within the polymer matrix, form a network that may act as a "scaffolding" to increase the film's modulus, and form conduction paths throughout the film that can reduce the internal resistance of the film leading to a demonstrated shorter voltage lifetime and higher power output. ${ }^{53}$

There are few studies that investigate piezoelectric nanocomposites made with $\mathrm{P}(\mathrm{VDF}$ TrFE) and CNT. One such study demonstrated that the addition of both SWCNT and MWCNT 
resulted in an increase in output power from $0.84 \mathrm{mV}$ (plain $\mathrm{P}(\mathrm{VDF}-\mathrm{TrFE})$ to $6.45 \mathrm{mV}(0.5 \mathrm{wt} \%$ MWCNT) and $7.80 \mathrm{mV}(0.5 \mathrm{wt} \%$ SWCNT) from an increased Young's modulus in the composite films, which results in better mechanical to electrical energy transfer. ${ }^{54}$ Another concurrent study reported that the increase to $1 \mathrm{wt} \%$ SWCNT loading in a P(VDF-TrFE) film resulted in an increase in piezoelectric as well as an increased output power due to interfacial polarization between the highly conductive CNT phase and the dielectric polymer matrix. ${ }^{55}$

One common problem in the development of CNT nanocomposites is the formation of nanofiller aggregates due to their highly homogenous non-polar structure. The most common method for increasing CNT dispersion is through particle surface stabilization either using covalent functionalization with heterogeneous functional groups or non-covalent functionalization with a surfactant or polymer. ${ }^{52}$ Covalent functionalization of CNTs is achieved by introducing heteroatomic polar functional groups such as carboxylic acid or hydroxyl groups through the reaction of strong oxidizing acids with defect points on the sides and ends of the CNT structure.

Non-covalent surface functionalization techniques involve either the adsorption of small molecule amphiphilic surfactants or polymer grafting onto the surface of pristine CNTs to decrease interfacial tension between the filler surface and its surrounding without altering the particle's composition. If the goal is to use CNT to promote polymer crystal nucleation, covalent CNT surface functionalization is likely the preferred technique in order to avoid the introduction of complicated polymer-surfactant interactions. Also, polar functional groups on the surface of CNTs may help induce interfacial crystallization through intermolecular attractions.

Recent studies have reported the ability to harvest energy from nanocomposite PNGs without the need for a costly poling process. Although the phenomenon is not yet well defined, these "self-poling" PNGs have demonstrated significant energy harvesting capability, and few mechanisms have been attributed to their function.

One such mechanism, pressure-induced polarization, suggests that piezoelectric films with zero net remnent polarization may be poled by mechanical stress. ${ }^{56}$ This effect was suggested to be responsible for the observed $20 \mathrm{~V}$ output voltage and $1 \mu \mathrm{A} \mathrm{cm}{ }^{-2}$ output current density from a PNG developed through the incorporation of $\mathrm{ZnSnO}_{3}$ nanocubes into a polydimethylsiloxane matrix 
without external poling treatment. It was also suggested that an increase in $\mathrm{ZnSnO}_{3}$ filler loading increased the observed output power of the film due to the formation of an interfacial MaxwellWagner-Sillars polarization at the interface between the PDMS matrix and the nanofiller, leading to the separation of charges and thus spatial electretic polarization. ${ }^{57}$

Another reported mechanism contributing to self-poling is the formation of heterogeneous electrets. Polarized domains form from the accumulation of trapped charges in certain dielectric materials either within micropores, interfaces between crystalline/amorphous polymer, or interfaces between polymer/nanofiller. Charges that are injected into macroscopic pores of dielectric films or boundaries between crystalline/amorphous regions of dielectric polymer films can gather and form molecular dipoles. ${ }^{58}$ According to the Maxwell-Wagner Sillars principle, within polymer nanocomposites, a large disparity of conductivity between the highly dielectric polymer matrix and a conductive filler results in a charge accumulation within their interfaces. ${ }^{59}$ The high surface areato-volume ratio of nanofillers facilitates a large amount of interfacial interaction between particles and dielectric polymer, resulting in a significant volume of charge storage between these interfaces. As well for carbon nanotube PNC samples, micron sized domains of conductive nanofiller contain delocalized electrons which eliminate the need for charge injection.

For example, Mandal et al. developed a PNG that utilized heterogeneous trapped charges with in-situ synthesized platinum nanoparticles (PtNP) suspended in a porous $\mathrm{P}($ VDF-HFP) matrix to facilitate electretic polarization. The PNG demonstrated the generation of $18 \mathrm{~V}$ of open-circuit output voltage with $17.7 \mu \mathrm{A}$ short-circuit current with $4 \mathrm{MPA}$ of stress, attributed to electretic space charging within the pores of the film, resulting in a remnant polarization. Also, crystalline domains of electroactive $\beta$ and $y$ phases of $P(V D F-H F P)$ are suggested to be formed through the interfacial nucleation between the fluorine groups on the copolymer and the PtNP surface, stabilizing trapped charges. The energy harvesting capabilities of the film were demonstrated by powering multiple LEDs and charging different capacitors. ${ }^{12}$ 


\subsection{Motivation and Research Plan}

The overall goal of this project was to develop a piezoelectric polymer nanocomposite that is prepared with simple processing conditions while avoiding the "stretch and pole" processing steps. As well, the project aimed to gain a better understanding regarding how the addition of certain nanoparticles might influence the piezoelectric properties of various copolymers of PVDF. Systems were designed and tested with $\mathrm{P}(\mathrm{VDF}-\mathrm{HFP})$ the $\mathrm{P}(\mathrm{VDF}-\mathrm{TrFE})$ to compare energy harvesting capabilities of the prepared formulations. Three high aspect-ratio nanomaterials, $\mathrm{ZnO}$ NW, MWCNT-OH, and SWCNT-COOH were utilized in this project, and the effect of their addition in nanocomposites were characterized. 


\section{EXPERIMENTS AND METHODS}

\subsection{Materials}

High purity single walled carbon nanotubes (length: $5-30 \mu \mathrm{m}$, diameter: $1-2 \mathrm{~nm},>95 \%$ purity) were purchased from Sun Innovations, Inc and were covalently functionalized with carboxylic acid groups in order to better disperse them throughout the film. Short hydroxyl functionalized multi walled carbon nanotubes (Length: $0.5-2 \mu \mathrm{m}$, diameter: 8-15 nm) were purchased from Cheaptubes and were used as received. P(VDF-HFP) with an average molecular weight $(\mathrm{Mw})$ of $455,000 \mathrm{~g} \mathrm{~mol}^{-1}$ was purchased in pellet form from Sigma-Aldrich. P(VDF-TrFE), 30 mol \% TrFE (Solvene 300/P300), average molecular weight $\left(\mathrm{Mw}_{\mathrm{w}}\right)$ of $300,000 \mathrm{~g} \mathrm{~mol}^{-1}$, was purchased as a powder from Sigma-Aldrich. Both polymers were used as received.

\subsection{Preparation of Nanoparticle Filler}

\subsubsection{Synthesis of Zinc Oxide Nanowires}

Zinc oxide nanowires were synthesized following previously reported solvothermal technique. ${ }^{45}$ To prepare the solution, $0.297 \mathrm{~g}$ zinc nitrate hexahydrate powder is added to $200 \mathrm{~mL}$ ethanol at room temperature. Powdered sodium hydroxide $(1.2 \mathrm{~g})$ was then added and mixed by vigorous stirring for 1 hour. The mixture was sonicated for an additional 30 minutes in a sonication bath to ensure that all base is dissolved. Ethylenediamine $(10 \mathrm{~mL})$ was added as a capping agent and the mixture was transferred to a Teflon lined vessel which was sealed in a Parr 4748 stainless steel autoclave to react at $130^{\circ} \mathrm{C}$ for 24 hours. Synthesized nanowires were collected from the dispersion by centrifugation, washed with water and ethanol several times, and dried in an oven.

\subsubsection{Functionalization of Single Walled Carbon Nanotubes}

Single walled carbon nanotubes were added to a 3:1 ratio of concentrated sulfuric acid and nitric acid, respectively in a sealed vial and sonicated in an ultrasonic bath for 4 hours to introduce carboxylic acid functional groups to the surface of the nanotubes (Figure 17). Functionalized nanotubes were isolated using vacuum filtration with a Teflon filter. The isolated NPs were rinsed with water and ethanol and then left to dry in a desiccator overnight. 

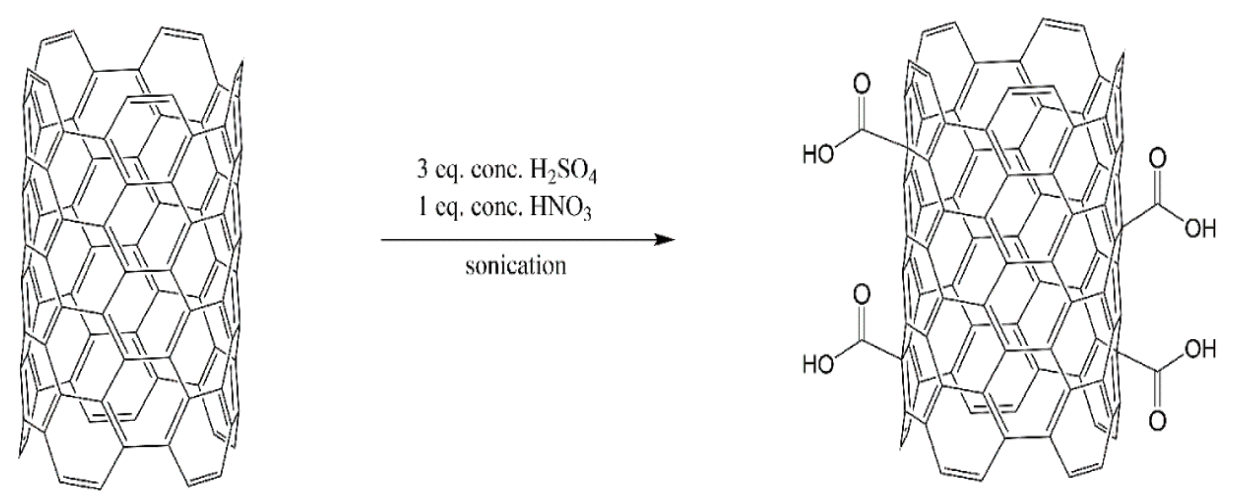

Figure 17. Acid functionalization of carbon nanotubes which introduces polar groups to the surface of CNTs.

\subsection{Piezoelectric Nanogenerator Preparation}

\subsubsection{P(VDF-HFP) Sample Preparation}

A 15 wt \% polymer stock was prepared by dissolving P(VDF-HFP) in dimethylformamide (DMF) under reflux for 3 hours. The mixed solution was then filtered using a $10 \mu \mathrm{m}$ pore size syringe filter to remove any impurities. To prepare PNC formulations, the mass of MWCNT-OH and ZnO NW filler were measured out corresponding to the sample's formulation (Table 1) and were added to a predetermined weight of copolymer solution. Resulting formulations were vortexed for 30 seconds and subsequently sonicated for 3 hours in a sonication bath.

Table 1. Filler Loading in 10 wt \% P(VDF-HFP) in DMF

\begin{tabular}{|c|c|c|}
\hline Sample & Weight Percent MWCNT-OH & Weight Percent ZnO NW \\
\hline C & 0 & 0 \\
\hline MZO & 1 & 0 \\
\hline MZ4 & 1 & 4 \\
\hline MZ8 & 1 & 8 \\
\hline
\end{tabular}


Films were prepared by casting using an adjustable doctor blade set either $100 \mu \mathrm{m}$ or 200 $\mu \mathrm{m}$ above a cleaned glass slide. Casted films were transferred to an oven at $80{ }^{\circ} \mathrm{C}$ to evaporate solvent.

\subsubsection{P(VDF-TrFE) Sample Preparation}

A 15 wt \% polymer stock solution of $\mathrm{P}(\mathrm{VDF}-\mathrm{TrFE})$ in DMF was prepared by dissolving $\mathrm{P}(\mathrm{VDF}-\mathrm{TrFE})$ in DMF under stirring at room temperature overnight. The mixed solution was then filtered using a $10 \mu \mathrm{m}$ pore size syringe filter to remove any impurities. Stock filler dispersions in DMF were prepared by suspending 0.5 wt $\%$ filler (either ZnO NW, MWCNT-OH, or SWCNT$\mathrm{COOH}$ ) in DMF. The dispersion is vortexed for 10 seconds and then sonicated in a bath sonicator for $\approx 3$ hours. Formulations of $0.1,0.5$ and $1 \mathrm{wt} \%$ filler were prepared by adding the desired amount of filler dispersion into $2 \mathrm{~g} 15 \mathrm{wt} \%$ polymer solution and then diluting with DMF to obtain the desired concentration of filler in 10 wt \% $\mathrm{P}(\mathrm{VDF}-\mathrm{TrFE})$. The mixture was vortexed for 30 seconds, bath sonicated for 6 hours, and then pulsed with probe sonication at $30 \%$ amplitude for 1 minute.

Films were prepared by casting formulations using an adjustable doctor blade set either $100 \mu \mathrm{m}$ or $200 \mu \mathrm{m}$ onto a cleaned glass slide. Casted films were transferred to an oven set at 60 ${ }^{\circ} \mathrm{C}$ to evaporate solvent and were subsequently annealed at $135^{\circ} \mathrm{C}$ for 3 hours. This annealing step has been shown to help induce growth of larger ordered crystal lamellae and has been established as an important step in enhancing the piezoelectric performance of PVDF-TrFE films. ${ }^{60}$ 
Table 2. Dimensions of Nanoparticles Used in the Study

\begin{tabular}{lccc}
\hline \multicolumn{1}{c}{ Nanoparticle } & Length $(\mathrm{nm})$ & Diameter $(\mathrm{nm})$ & Average Aspect Ratio (L/D) \\
\hline ZnO NW & $1245 \pm 725$ & $26.5 \pm 7.0$ & 47 \\
MWCNT-OH & $1250 \pm 750$ & $11.5 \pm 3.5$ & 109 \\
SWCNT-COOH & $1750 \pm 125$ & $1.5 \pm 0.5$ & 1,167 \\
\hline * Dimensions reported are from supplier of pristine SWNCT - acid functionalization \\
\multicolumn{2}{l}{ likely leads to shortened CNT and increased particle polydispersity }
\end{tabular}

\subsubsection{Film Thickness Determination}

Films were cast at $100 \mu \mathrm{m}$ and $200 \mu \mathrm{m}$ in triplicate for each formulation for thickness measurements. The thickness of each film was measured around 10 times throughout the area of the film using a Mituyoyo 0-1" range digital micrometer. Multiple measurements were taken and averaged to provide a general average thickness of formulations.

\subsection{Instrumental Methods}

\subsubsection{Scanning Electron Microscopy}

The morphology of ZnO NW was characterized using the JEOL JSM-7610F scanning electron microscope (SEM) operated at an acceleration voltage of $3.0 \mathrm{kV}$, WD $7.9 \mathrm{~mm}$. The sizes of individual nanowires were measured from SEM images using ImageJ image processing software. ${ }^{61}$ To prepare for SEM, the nanowires were added to a vial with $\mathrm{EtOH}$ and dispersed through bath sonication. The resulting suspension was transferred onto a clean silicon substrate and solvent was evaporated at room temperature. The sample was then applied to a carbon tapecovered SEM sample holder.

\subsubsection{X-Ray Diffraction}

$\mathrm{X}$-ray diffraction $(\mathrm{XRD})$ is a powerful tool used to gain information on the crystal structure of various materials. The technique takes advantage of the fact that the regularity of spacing planes within crystal structures behave like that of a three-dimensional diffraction grating. When filtered monochromatic X-ray radiation interacts with a regularly spaced crystal lattice, the $\mathrm{X}$-rays are 
scattered to form regular diffraction patterns. Information is reconstructed from these patterns to obtain information about the sample's crystal structure.

XRD measurements were performed on a Bruker AXS D8 ADVANCE X-ray diffractometer equipped with a LynxEye 1-dimensional linear Si strip detector. The samples were scanned from 10 to 80 degrees $2 \theta$. The step scan parameters were 0.02 degree steps and 2 seconds counting time per step with a 0.499 degree divergence slit and a 0.499 degree antiscatter slit. The X-ray source was Ni-filtered Cu radiation from a sealed tube operated at $40 \mathrm{kV}$ and $40 \mathrm{~mA}$. Phases in the samples were identified by comparison of observed peaks to those in the International Centre for Diffraction Data (ICDD PDF2018) powder diffraction database.

\subsubsection{Fourier-Transform Infrared Spectroscopy}

Fourier-transform infrared spectroscopy (FTIR) was employed to characterize the presence of crystal polymorphs within the copolymer films. An OPUS Platinum Alpha Fouriertransform infrared spectrometer was used in ATR mode from 4,000 to $400 \mathrm{~cm}^{-1}$. FTIR spectra were collected with 24 scans and a resolution of $4 \mathrm{~cm}^{-1}$. Distinct vibrational modes within the spectra is attributed to variation in crystal packing depending on a polymorph's chain orientation. Assuming the FTIR absorption follows the Beer-Lambert law, the fraction of beta phase $F(\beta)$ is determined with FTIR using the following equation:

$$
F(\beta)=\frac{A_{\beta}}{(1.26) A_{\alpha}+A_{\beta}}
$$

Where $\mathrm{A}_{\alpha}$ corresponds to the $\mathrm{CF}_{2}$ bending and skeletal bending vibrational mode of the alpha phase at $763 \mathrm{~cm}^{-1}$, absorbance $A_{\beta}$ corresponds to the $\mathrm{CH}_{2}$ rocking vibrational mode of the beta phase at $840 \mathrm{~cm}^{-1}$, and 1.26 is the ratio of absorption coefficient corresponding to the $\beta$-phase $\left(\mathrm{K}_{\beta}=7.7 \times 10^{4} \mathrm{~cm}^{2} / \mathrm{mol}\right)$ relative to $\alpha$-phase $\left(\mathrm{K}_{\alpha}=6.1 \times 10^{4} \mathrm{~cm}^{2} / \mathrm{mol}\right) .62,63$

\subsubsection{Differential Scanning Calorimetry}

Differential Scanning Calorimetry (DSC) was utilized as a useful instrument to glean information on the thermodynamic properties of the polymer films. In a typical run, a pan with sample in it and reference pan left empty are loaded into heaters that maintain identical temperatures. Phase transitions within the sample either absorb or release heat, requiring a 
difference in power supplied to the two heaters to maintain matching temperatures. The difference in power supplied to the heaters as a function of temperature is maintained in the final graph. A first order transition such as melting will display an endothermic peak in the DSC signal, and the temperature corresponding to the maximum of that peak represents the melting temperature $\left(T_{m}\right)$. The enthalpy of fusion $\left(\Delta H_{f}\right)$ is calculated with following equation:

$$
\Delta H_{f}=\int_{T_{1}}^{T_{2}} C_{p} d T
$$

Equation 8 is equivalent to the integral of the endothermic melting peak within the DSC signal. The degree of crystallinity $\left(\chi_{c}\right)$ of a semi-crystalline polymer is determined with the measured enthalpy data and the melting enthalpy of completely crystalline polymer:

$$
\chi_{c}=\frac{\Delta H_{f}}{\Delta H_{f}^{0}}
$$

Where the enthalpy of completely crystalline $\mathrm{P}(\mathrm{VDF}-\mathrm{TrFE})$ is $\Delta H_{f}^{0}=91.45 \mathrm{~J} / \mathrm{g} .{ }^{64}$

DSC measurements were performed on a TA Instruments Q1000 differential scanning calorimeter equipped with a 50-position auto sampler. Sealed aluminum hermetic pans were used to contain samples and for reference pans. A sample cut from each film, around $5 \mathrm{mg}$, was used for each run. The samples were initially melted at $180^{\circ} \mathrm{C}$ and maintained at this temperature for 5 minutes to erase any thermal history. Samples were then cooled from $180^{\circ} \mathrm{C}$ to $25^{\circ} \mathrm{C}$ at a rate of $5^{\circ} \mathrm{C} \min ^{-1}$ and subsequently heated back to $180^{\circ} \mathrm{C}$ at a rate of $5^{\circ} \mathrm{C} \mathrm{min}^{-1}$.

\subsubsection{Polarized Optical Microscopy}

Highly aligned domains of crystalline polymer have special optical properties such as birefringence that is assessed using techniques such as polarized optical microscopy (POM). Birefringence is a result of anisotropic structure of the lamellar crystals, which result in a difference between refractive indices in two planes. During transition mode POM, light from a lamp source passes through a polarizer, the sample, a second polarizer positioned 90 degrees rotated in relation to the first, and then a microscope camera. With a non-birefringent sample, the incident polarized light cannot pass through the second perpendicular polarizer, and thus no light is observed in the image. When a birefringent polymer crystal is placed between the polarizer, the plane polarized light interacts with the anisotropic crystal, and two perpendicular light components known as the 
ordinary and extraordinary light waves result. When the polarized light waves enter through the second polarizer, only those that are parallel to the polarizer pass through, and are observed in the image. Thus, highly aligned polymer crystalline structures are observed through this technique. Images of $\mathrm{P}(\mathrm{VDF}-\mathrm{TrFE})$ nanocomposite films were taken with the Nikon Eclipse polarized optical microscope under $60 x$ magnification with $90^{\circ}$ crossed polarizers. A DS-Fi1 camera was used to capture images of crystalline nanocomposite films.

\subsubsection{Atomic Force Microscopy}

Atomic force microscopy (AFM) is an advanced imaging technique that can be used to characterize the surface morphology of PNC films which helps to provide information about the effect of nanofiller addition on the polymer crystal microstructure. The working mechanism of AFM involves sweeping of a cantilever with a sharp tip that is raster-scanned over a sample surface. Atomic interactions between the cantilever tip and the surface results in a deflective force that is measured by a Z-servo feedback loop used to adjust the height of the tip to control the tip-sample distance. Cantilever deflection is measured by the detection of a laser beam reflected off of the cantilever into a position-sensitive diode. The change in measured deflection is translated into a topography map which depicts the surface microstructure. Tapping AFM is a mode of AFM that oscillates the tip in the z-axis as it runs along the substrate surface near the cantilever's resonant frequency. This method overcomes problems that come from dragging the tip across the surface such as friction or adhesion that could lead to image artifacts.

AFM images were obtained using tapping mode on the Asylum Research Molecular Force Probe 3D (MFP-3D) microscope under ambient conditions. Commercial silicon cantilevers with force constants of $12-70 \mathrm{~N} \mathrm{~m}^{-1}$ and fundamental resonance frequencies of $160 \mathrm{kHz}$ were used for imaging. Topographic images of each sample were analyzed using the Gwyddion Scanning Probe Microscopy (SPM) open-source software. ${ }^{65}$ 


\subsubsection{Device Fabrication}

Piezoelectric nanogenerators were dried films by the application of copper electrodes to either side of the film, with protruding off-set strips for direct oscilloscope lead attachment. The films were then encapsulated in SYLGARD 184 Silicone (Dow) using a 10:1 ratio of elastomer to curing agent to prevent structural damage to the devices and to facilitate uniform force distribution throughout the devices. Initial P(VDF-HFP) and P(VDF-TrFE) devices were prepared with $25.5 \mathrm{x}$ $17 \mathrm{~mm}$ electrodes, whereas later versions of $\mathrm{P}(\mathrm{VDF}-\mathrm{TrFE})$ devices were prepared with $10 \times 35 \mathrm{~mm}$ electrodes.

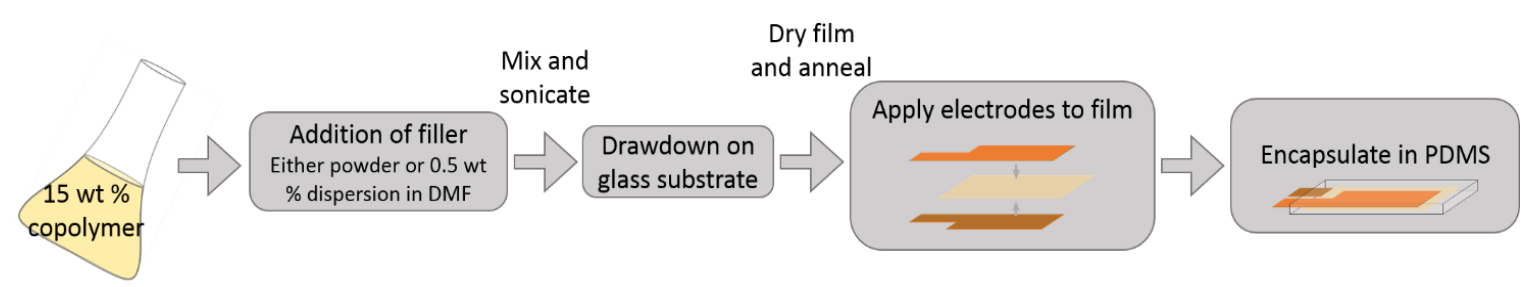

Figure 18. Method for piezoelectric nanogenerator device fabrication.

Alternative electrode designs were explored to optimize device flexibility and processing conditions. Devices were prepared with electrodes made from DuPont silver epoxy paste diluted to a 1:1 ratio with butyl acetate through airbrush spray application using a steel stencil with a $10 \times 35$ $\mathrm{mm}$ patterned opening. The resulting electrodes had a low resistance $(104 \mathrm{~m} \Omega / \mathrm{sq})$ and excellent flexibility. Another electrode design was also explored through screen printing with the conductive polymer mixture poly(3,4-ethylenedioxythiophene) polystyrene sulfonate (PEDOT:PSS) (SigmaAldrich). To apply this electrode, the polymer film was fixed to the bench surface and a mesh screen was used to print the PEDOT:PSS onto the film. The electrode was dried at $80^{\circ} \mathrm{C}$, and another coating was applied to the other side of the film and dried. Copper wire leads were applied to the electrodes using silver paste and carbon tape. Sheet resistance was measured using the Keithley Instrument 2400 SourceMeter with a 4-point probe configuration to compare quality of electrode materials. 


\subsubsection{Power Testing}

Film energy harvesting performance was determined through a series of strain tests using a digital oscilloscope. Initial tests with P(VDF-HFP) PNGs were performed with hand tapping tests with compression normal to the film. Tests to compare the output power of $P(V D F-T r F E)$ were carried out using Instron 5943 equipped with a $1 \mathrm{kN}$ load cell and cylindrical compression platens. PNG devices were adhered to a stabilized platform on the bottom platen using double sided tape and the top platen was offset from the sample surface by $1.6 \mathrm{~mm}$ to simulate a "tapping" force onto the device. Cyclic compression was run with an extension of $1.6 \mathrm{~mm}$ at a rate of $18 \mathrm{~mm} / \mathrm{s}$ for 10 cycles.

Output voltage was measured using a Tektronix TDS 2014C digital oscilloscope directly connected to piezoelectric devices. Output current was measured by recording the voltage drop from a shunt resistor setup using $1 \mathrm{M} \Omega$ resistor in parallel with the device. The current was

extrapolated by using Ohm's law $\left(I=\frac{V}{R}\right)$ with the known value of the resistor and the output voltage recorded.

The circuit for a standard PNG energy harvesting device is seen in Figure 19. A full wave bridge rectifier consists of four diodes arranged as a bridge so that both positive and negative electrical pulses from the PNG are directed towards the same positive terminal and is used to convert AC power from the PNG to DC. The rectified power is stored within a capacitor, and a single pole double throw (SPDT) switch is used to transfer power stored within the capacitor to some load, in this case a display. 

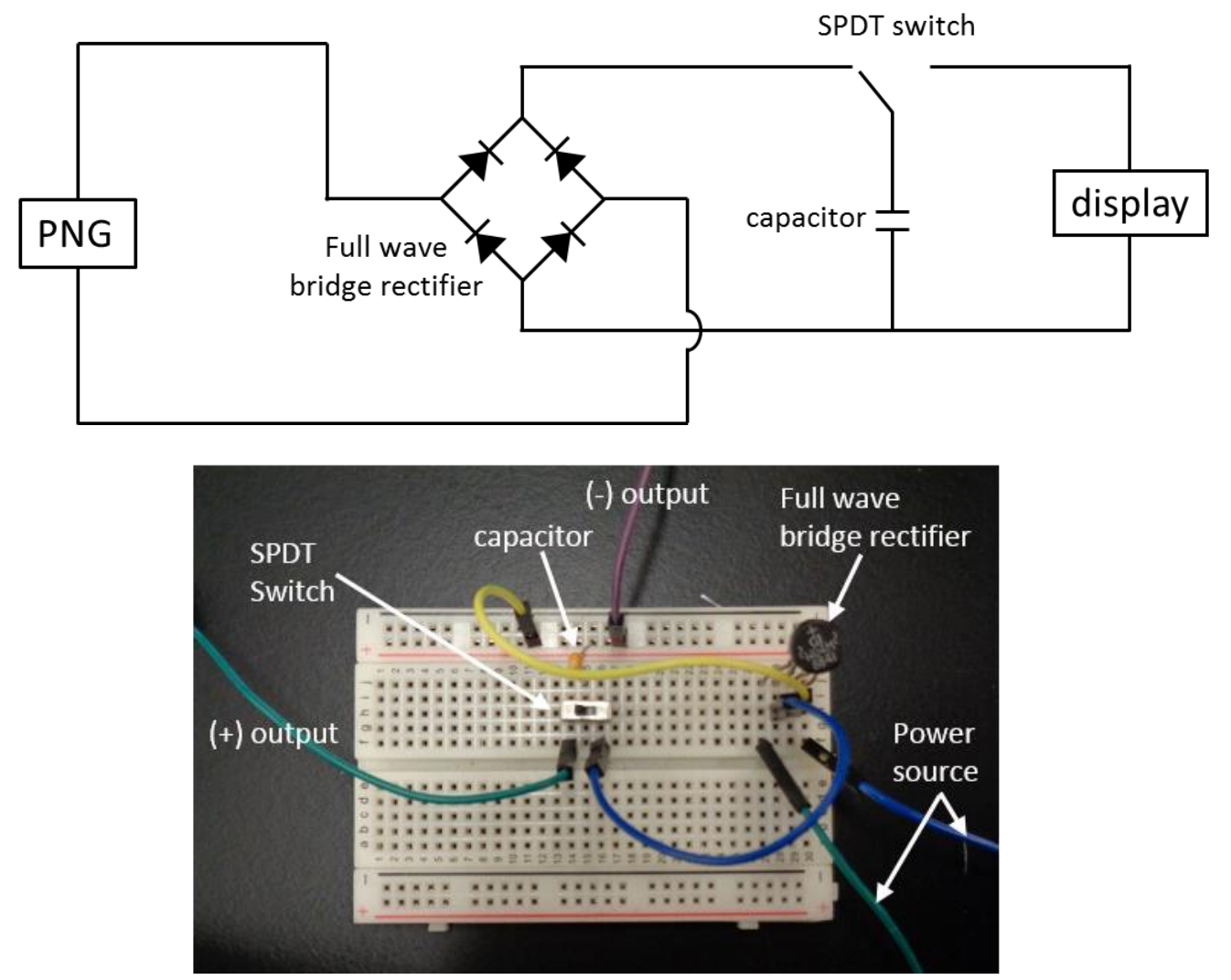

Figure 19. (Top) circuit used for power harvesting of piezoelectric nanogenerator (PNG) (Bottom) Actual power harvesting circuit on breadboard.

The initial PNG energy harvesting device was set up using a breadboard, and later models were designed as integrated circuits for portable energy harvesting devices. A $10 \mathrm{nF}$ capacitor was chosen as the energy storage capacitor. 


\section{RESULTS AND DISCUSSION}

\subsection{Preparation of Nanoparticle Filler}

\subsubsection{Zinc Oxide Nanowires}

Synthesized zinc oxide nanowires ( $\mathrm{nO} \mathrm{NW}$ ) were characterized to confirm their morphology and crystal structure. Using SEM, the synthesized nanomaterials showed the expected high aspect ratio wire-like morphology, with a measured average diameter of $26.5 \pm 7.0 \mathrm{~nm}$, average length of $1245 \pm 725 \mathrm{~nm}$, and an average aspect ratio (L/D) of 47 .
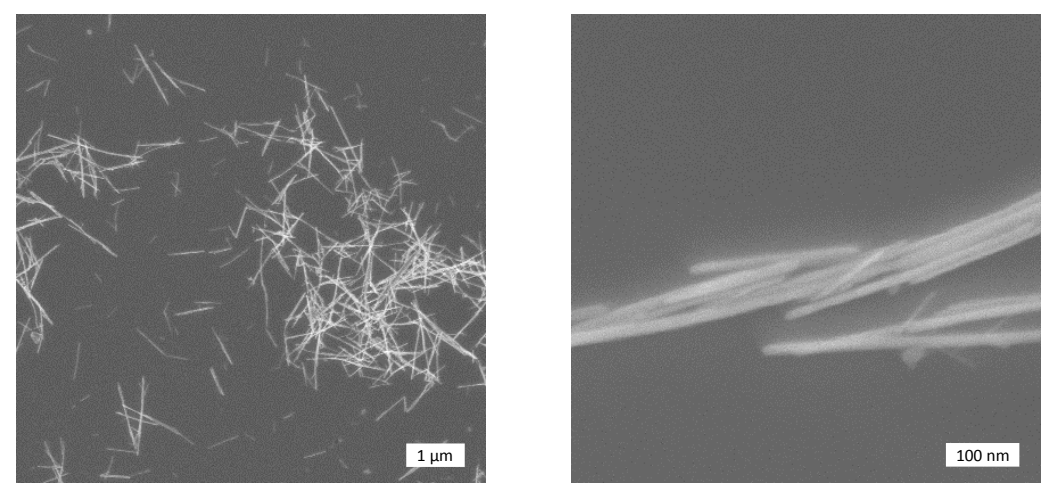

Figure 20. SEM images of synthesized ZnO NW, where images show (left) dispersion of ZnO NW $(x 5,500)$ and (right) zoomed in depiction of $\mathrm{ZnO}$ NW diameters $(x 90,000)$.

X-ray diffraction (XRD) spectra of dispersed ZnO NW powder confirms that synthesized $\mathrm{ZnO}$ NW are a hexagonal wurtzite structure which has non-centrosymmetric polarization along its c-axis with lattice parameters of $\mathrm{a}=3.25 \AA$ and $\mathrm{c}=5.21 \AA$ confirmed by comparison with PDF 01 070-8070 card (Appendix Figure A.1). UV-Vis spectroscopy of ZnO NW prepared in water showed peak absorption at $367 \mathrm{~nm}$ agreeing with expected band gap of $3.37 \mathrm{eV}$ (Appendix Figure A.2). ${ }^{45}$ 


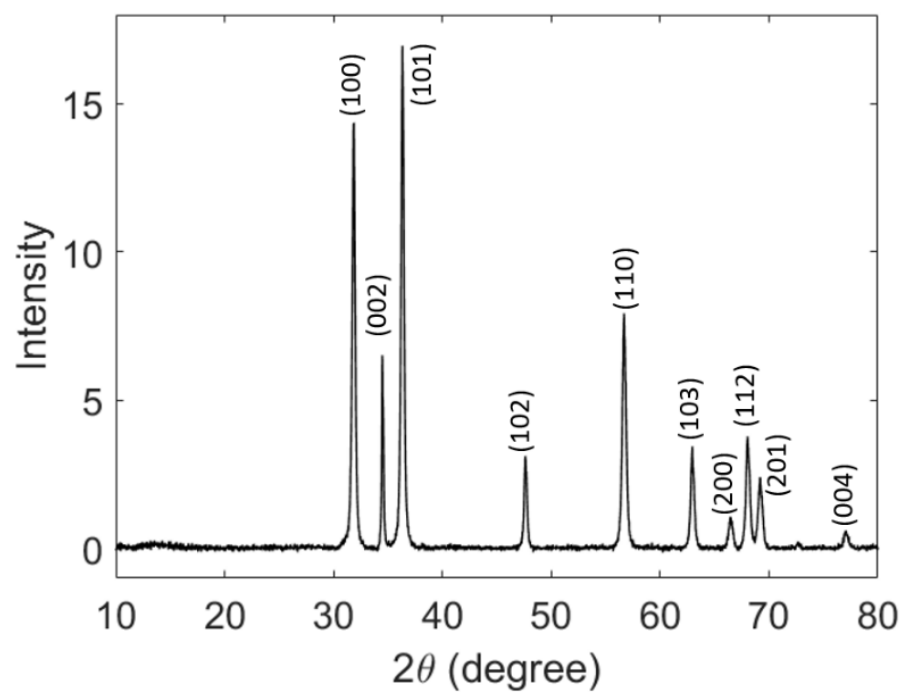

Figure 21. XRD diffractogram of solvothermally grown $\mathrm{ZnO} N W$ confirming non-centrosymmetric hexagonal wurtzite structure with lattice parameters of $a=3.25 \AA$ and $c=5.21 \AA$.

\subsection{P(VDF-HFP) Nanocomposites}

\subsubsection{Film Crystalline Properties}

The crystalline morphology of the $\mathrm{P}(\mathrm{VDF}-\mathrm{HFP})$ nanocomposites were characterized through FTIR spectroscopy. Using Equation 7, the percentage of $\beta$-phase $(F(\beta) \times 100 \%)$ of the control $\mathrm{P}(\mathrm{VDF}-\mathrm{HFP})$ without added nanoparticle filler was determined to be $56 \%$. In formulation MZO (1 wt \% MWCNT-OH), the amount of $\beta$-phase in composite films increased up to $82 \% \pm 1 \%$ for $100 \mu \mathrm{m}$ wet films and $78 \% \pm 3 \%$ in $200 \mu \mathrm{m}$ wet films. In formulation MZ4 (1 wt \% MWCNT-OH and $4 \mathrm{wt} \% \mathrm{ZnO} \mathrm{NW}$ ), the amount of $\beta$-phase was $80 \% \pm 1 \%$ for $100 \mu \mathrm{m}$ wet films and $81 \% \pm 1 \%$ in $200 \mu \mathrm{m}$ wet films. As well, in formulation MZ8 (1 wt \% MWCNT-OH and 8 wt \% ZnO NW), the amount $\beta$-phase was $79 \% \pm 1 \%$ for $100 \mu \mathrm{m}$ wet films and $81 \% \pm 3 \%$ in $200 \mu \mathrm{m}$ wet films. These results show that the addition of just 1 wt $\%$ MWCNT-OH leads to an increase in the relative amount of $\beta$-phase as high as $26 \%$, whereas further addition of $\mathrm{ZnO} \mathrm{NW}$ does not lead to any significant change in the amount of $\beta$-phase. 
(a)

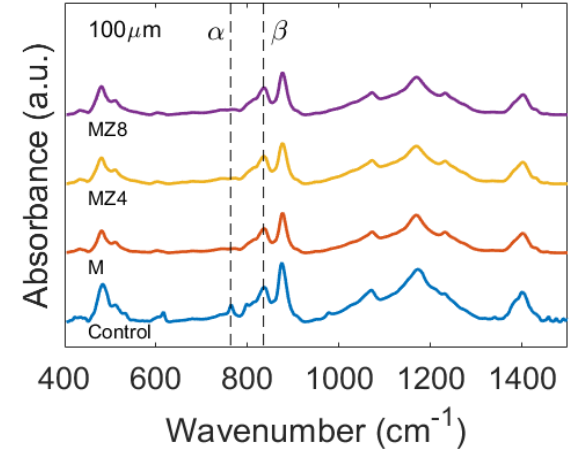

(b)
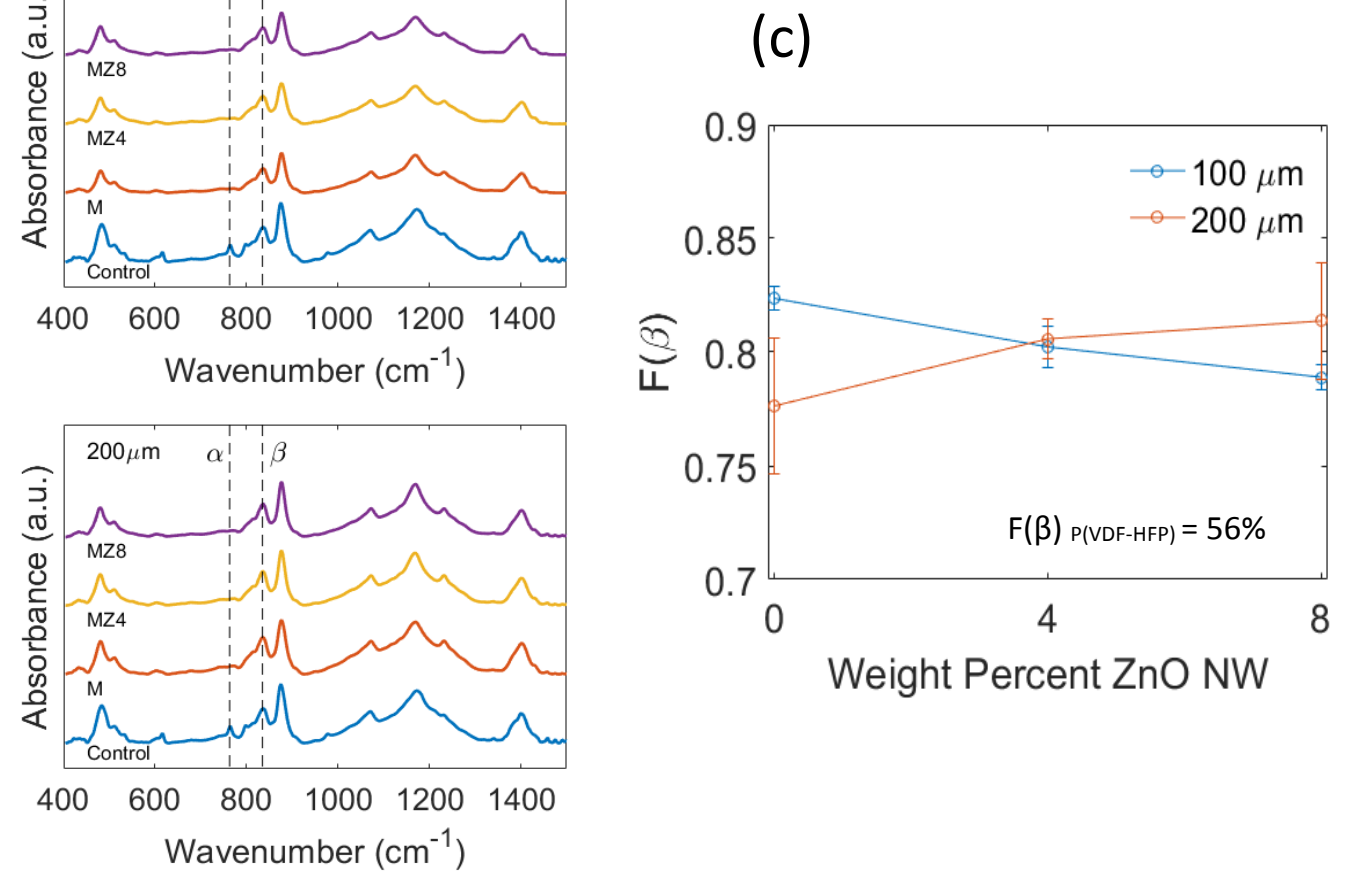

Figure 22. Results from FTIR study (a) FTIR spectra of $100 \mu \mathrm{m}$ wet cast (b) FTIR spectra of 200 $\mu \mathrm{m}$ wet cast (c) Fraction of beta phase $\mathrm{F}(\beta)$ of $\mathrm{P}(\mathrm{VDF}-\mathrm{HFP})$ copolymer composites with 1 wt \% MWCNT-OH and a range of 0,4 , and 8 wt $\% \mathrm{ZnO} \mathrm{NW}$ casted at $100 \mu \mathrm{m}$ and $200 \mu \mathrm{m}$ wet films.

\subsubsection{Power Harvesting}

The power harvesting properties of the P(VDF-HFP) PNGs were tested with a hand tapping test imparted on devices made from $100 \mu \mathrm{m}$ wet cast films. Results from tap testing (Figure 23) show that sample $C$ (pure $P\left(\right.$ VDF-HFP) film) exhibits an average voltage $V_{\text {ave }}=140 \mathrm{mV}$. In comparison, sample $M Z 0$ had a $V_{a v e}=0.74$ and $M Z 8$ had a $V_{\text {ave }}=0.98 \mathrm{~V}$. These results suggest that the addition of just 1 wt $\%$ MWCNT-OH increase the output voltage by $\sim 5 x$. Subsequent addition of $8 \mathrm{wt} \% \mathrm{ZnO} \mathrm{NW}$ shows a minimal increase in average output voltage not nearly as significantly as the addition of the $1 \mathrm{wt} \% \mathrm{MWCNT}-\mathrm{OH}$, following the trend similar to that of the increase in $\beta$-phase observed in FTIR results. 


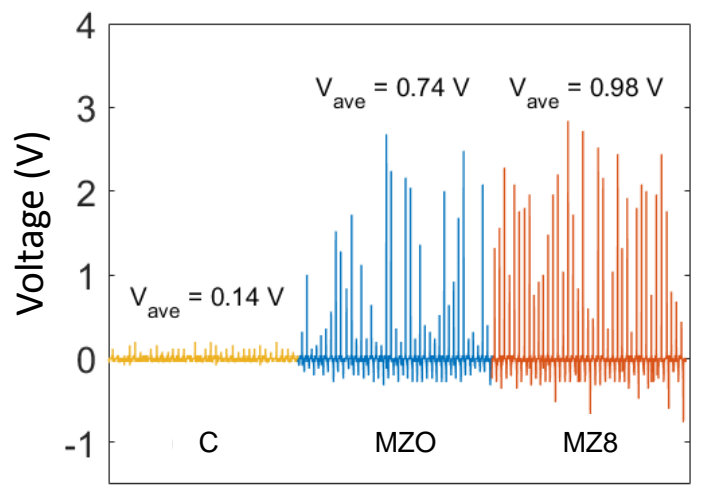

Figure 23. Output voltage of $\mathrm{P}(\mathrm{VDF}-\mathrm{HFP})$ formulations from tapping demonstrating an increase in increase in voltage with the addition of nanoparticle filler.

Further tests must be run in order to confirm the magnitude of the increase in output power demonstrated from this test. Also, it is important to test the repeatability of these results with a method that has a standardized application force and frequency.

To explain the increase in output power from the addition of nanofiller into P(VDF-HFP) films, a few mechanisms are considered. First, a significant increase in electroactive $\beta$-phase of around $26 \%$ was observed, increasing the volume of polar crystal domains within the films. This increase in $\beta$-phase content would be a clear explanation for an increase in output device power if the films had been poled to align polar domains, but because these samples were not poled, the explanation for a power increase becomes more complicated. For this system, a deeper understanding of the mechanisms behind the power generation is needed.

\subsection{P(VDF-TrFE) Nanocomposites}

Following the results from the P(VDF-HFP) nanocomposite formulations, PNCs with $\mathrm{P}($ VDF-TrFE) copolymer and various nanoparticle fillers were prepared to attempt to achieve an increase in output power. Single-filler formulations were prepared with varying weight percent of ZnO NW, MWCNT-OH, and SWCNT-COOH, and a more comprehensive combination of tests were run to investigate possible mechanisms responsible for the observed increase in the PNG's piezoelectric properties. 


\subsubsection{Film Structure}

In PNGs, film thickness is optimized to produce a desired output signal while accounting for limitations for the film's final application and maintaining mechanical durability. Polymer piezoelectric applications often take advantage of the low thickness and high flexibility in the devices, which result in a small cross-sectional area that experience large amounts of stress induced deformation when a relatively small force is applied. That said, there are a few limitations on decreasing film thickness. For example, thicker films typically generate higher voltages, and thinner films can experience issues such as pitting, which can result in a short between electrodes. ${ }^{66}$ Thus, care must be taken when designing a polymer PNG so that the film thickness is optimized to account for its application and proper function.

Films were characterized to determine the relationship between wet cast film thickness and that of the resulting dried film. It was found that the average thickness of films cast at $100 \mu \mathrm{m}$ wet were around $10 \mu \mathrm{m}$ when dried, which indicated that the dried films cast at this thickness end up about one-tenth the thickness of the wet cast. On the other hand, the average thicknesses of films cast at $200 \mu \mathrm{m}$ wet were around $15 \mu \mathrm{m}$ when dried, not following the one tenth thickness ratio previously observed. This lower than expected thickness is possibly due to wetting effects from the low viscosity formulation.

The addition of nanofiller generally shows an increase in dry film thickness in all cases except for an observed decrease in thickness upon the increase from 0.5 to $1 \mathrm{wt} \%$ filler. The $1 \mathrm{wt}$ $\% \mathrm{ZnO}$ NW dried films were only measured seven times, so more measurements are needed to account for uncertainty. The increase in film thicknesses might be caused by the increased stiffness of the PNC with the addition of rigid nanofiller, resulting in less deformation when stress from the micrometer is applied. Also, large nanofiller aggregates form within the thin film which may add to the film's overall volume. As seen in images from POM (Appendix Figure A.4), smaller CNT aggregates appear to be multiple micrometers in size, whereas large aggregates range up to hundreds of micrometers, visible by eye (Appendix Figure A.4). 

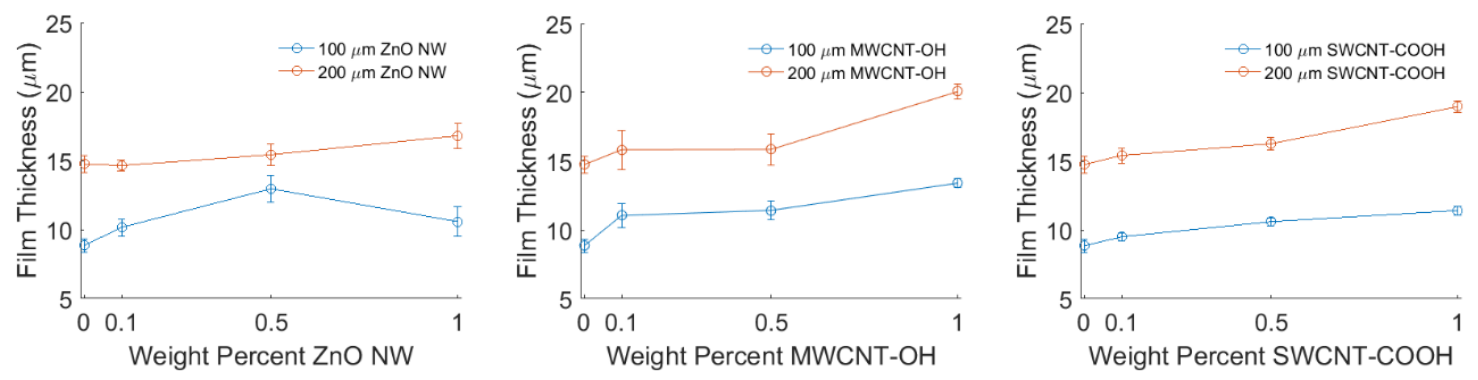

Figure 24. Film thickness of 100 and $200 \mu$ m wet cast films with $0,0.1,0.5$, or 1 wt \% nanofiller.

Suspending individual CNT particles within most solvents is difficult due to the homogeneity and strong nonpolar interactions throughout their structure. As previously mentioned, chemical surface functionalization and bath sonication can both significantly aid in forming a stable particle suspension, but CNT bundles on the microscale formed from strong interactions between the particles often still persist after these treatments. This problem is addressed through the application of high frequency vibrational forces in direct contact with the nanocomposite using a probe sonicator. This technique was employed on both $\mathrm{ZnO}$ NW and CNT formulations to ensure that samples had well suspended nanoparticle dispersions. A clear decrease in aggregate size can be observed between films that did not undergo probe sonication treatment and films that had been treated for one minute (Figure 25).
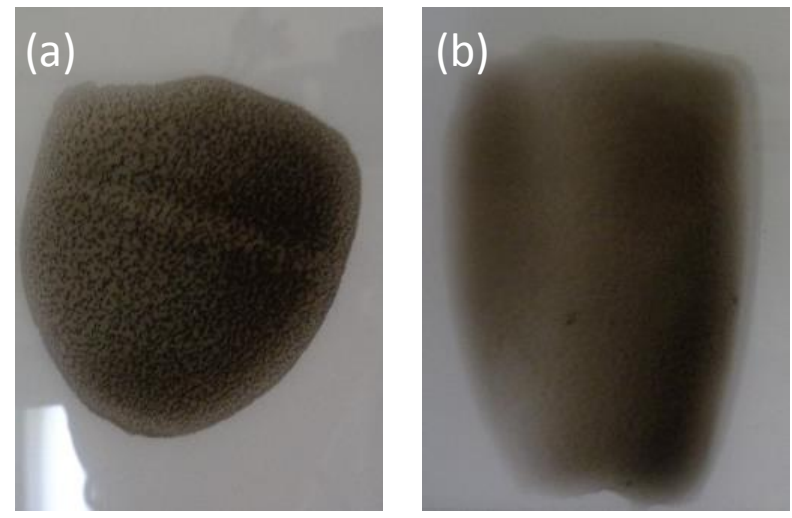

Figure 25. $200 \mu \mathrm{m}$ P(VDF-TrFE) films loaded with 1 wt \% SWCNT-COOH casted (a) before and (b) after 1 minute of pulsed probe sonication. 


\subsubsection{Film Crystalline Properties}

FTIR spectroscopy was used as a quick and informative method to analyze the effect of NP loading on the crystal structure of the P(VDF-TrFE) polymer. Bands at $508,840,1285$, and $1431 \mathrm{~cm}^{-1}$ correspond to the $\beta$-phase polymorph of the copolymer, whereas bands at 532,612 , $763,796,854,870$, and $970 \mathrm{~cm}^{-1}$ correspond to its $\alpha$-phase. ${ }^{67}$ The presence of $\beta$-phase bands and absence of $\alpha$-phase bands in FTIR spectra (Figure 26) indicates that crystalline domains within the films are mostly made up of the $\beta$-phase polymorph. Spectra also show that there is an insignificant change in the fraction of beta phase in both the $100 \mu \mathrm{m}$ and $200 \mu \mathrm{m}$ wet casted samples throughout all of the samples, indicating that the addition of nanofiller did not significantly affect the preferred crystal polymorph formation P(VDF-TrFE) copolymer.

FTIR spectroscopy can also be used to gather information on the orientation of crystal domains. Of these, peaks around $1200 \mathrm{~cm}^{-1}, 1290 \mathrm{~cm}^{-1}$, and $1400 \mathrm{~cm}^{-1}$ correspond to vibrational modes parallel to the a-axis, b-axis, and c-axis, respectively. ${ }^{68}$ The spectra show nearly identical bands at these peaks for all samples, indicating that the addition of nanofiller did not alter the orientation of the copolymer's crystal domains. 

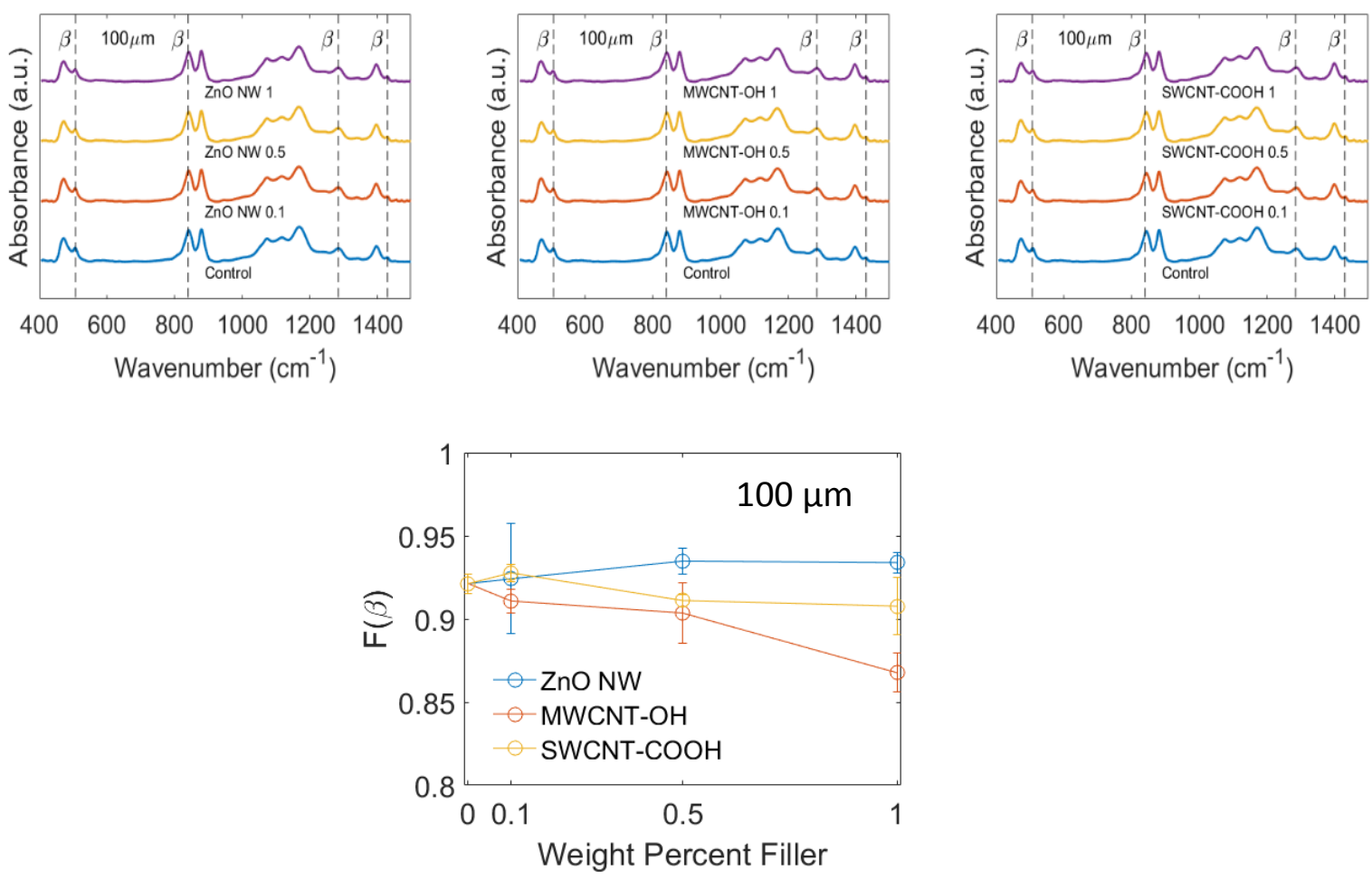

Figure 26. FTIR spectra of $100 \mu \mathrm{m}$ wet cast samples with the addition of $0.1,0.5$, or $1 \mathrm{wt} \%$ (left to right) $\mathrm{ZnO}$ NW, MWCNT-OH, or SWCNT-COOH.

Films cast at $200 \mu \mathrm{m}$ wet were also characterized with FTIR to determine if an increase in bulk phase has any effect in the crystalline properties of the copolymer. Again, no change in peak height corresponding to the $\alpha$ or $\beta$ phase was observed, indicating that the nanofiller addition had no effect on the fraction of $\beta$ phase present in the films. Additionally, peaks corresponding to aaxis, b-axis, and c-axis saw no peak change with the addition of nanofiller. 

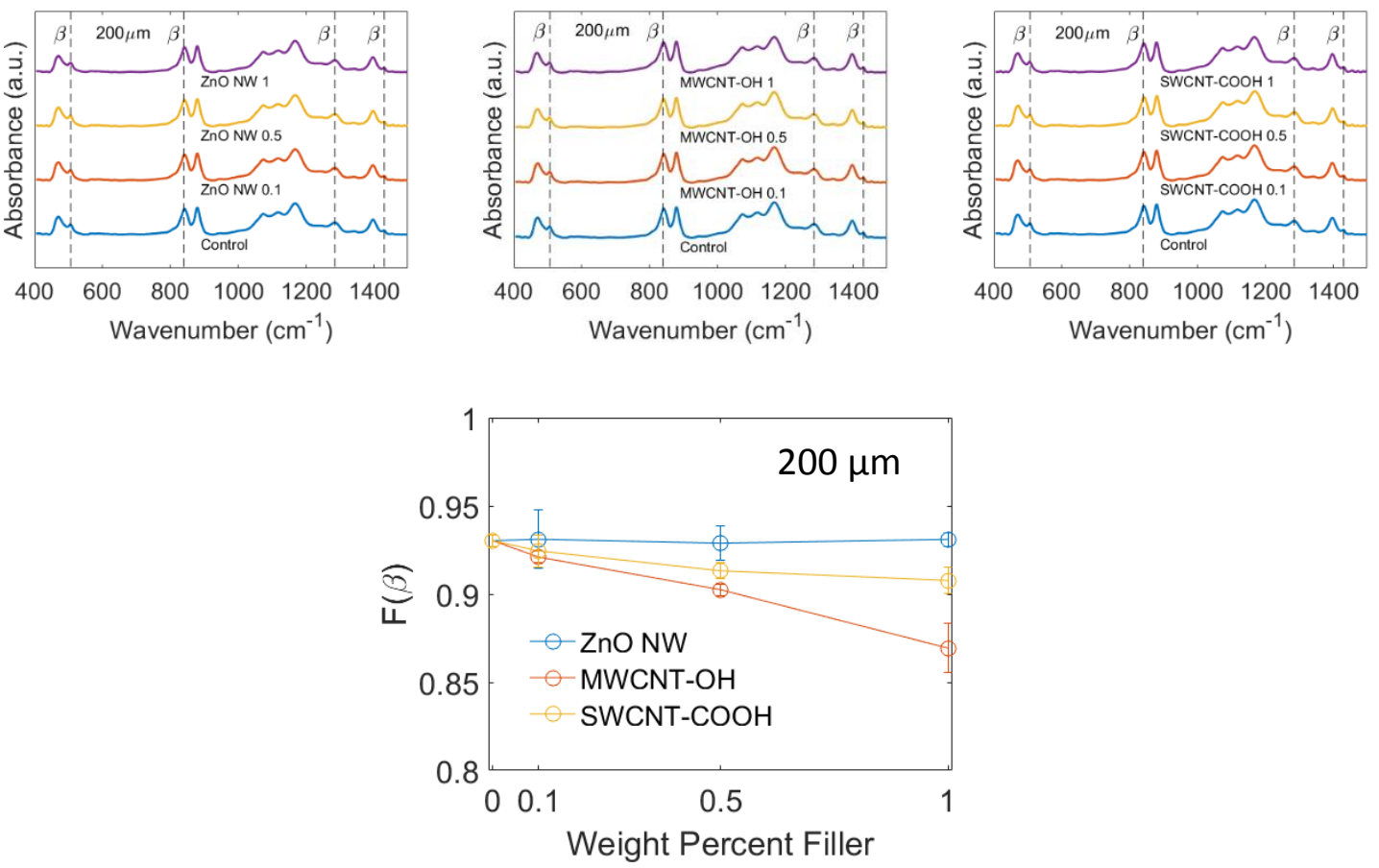

Figure 27. FTIR spectra of $200 \mu \mathrm{m}$ wet cast samples with the addition of $0.1,0.5$, or $1 \mathrm{wt} \%$ (left to right) $\mathrm{ZnO}$ NW, MWCNT-OH, or SWCNT-COOH.

XRD was employed to further assess the effect of NP addition on the preferred crystal polymorph formation of the $100 \mu \mathrm{m}$ wet cast PNGs. In P(VDF-TrFE) samples, peaks at $19.9^{\circ}, 35.2^{\circ}$, and $40.7^{\circ}$ which correspond to the $(200) /(110)$ planes, $(001) /(310) /(020)$ planes, and $(111) /(201) / 400) /(220)$ planes of the $\mathrm{P}\left(\right.$ VDF-TrFE) $\beta$-phase, respectively. ${ }^{4,69}$ The peak at $31.8^{\circ}$ in the ZnO NW PNGs is attributed to the $\mathrm{ZnO}(100)$ crystallographic plane which appears at the addition of $0.5 \mathrm{wt} \% \mathrm{ZnO} \mathrm{NW}$ and increases in a linear fashion as more nanofiller is added. Existence of these peaks and lack of $\alpha$-phase peak at $18.5^{\circ}$ suggests that all samples preferentially form the $\beta$-phase crystal polymorph. Observations agree with FT-IR data suggesting the addition of nanoparticle filler does not substantially effect the crystalline structure of the films. 

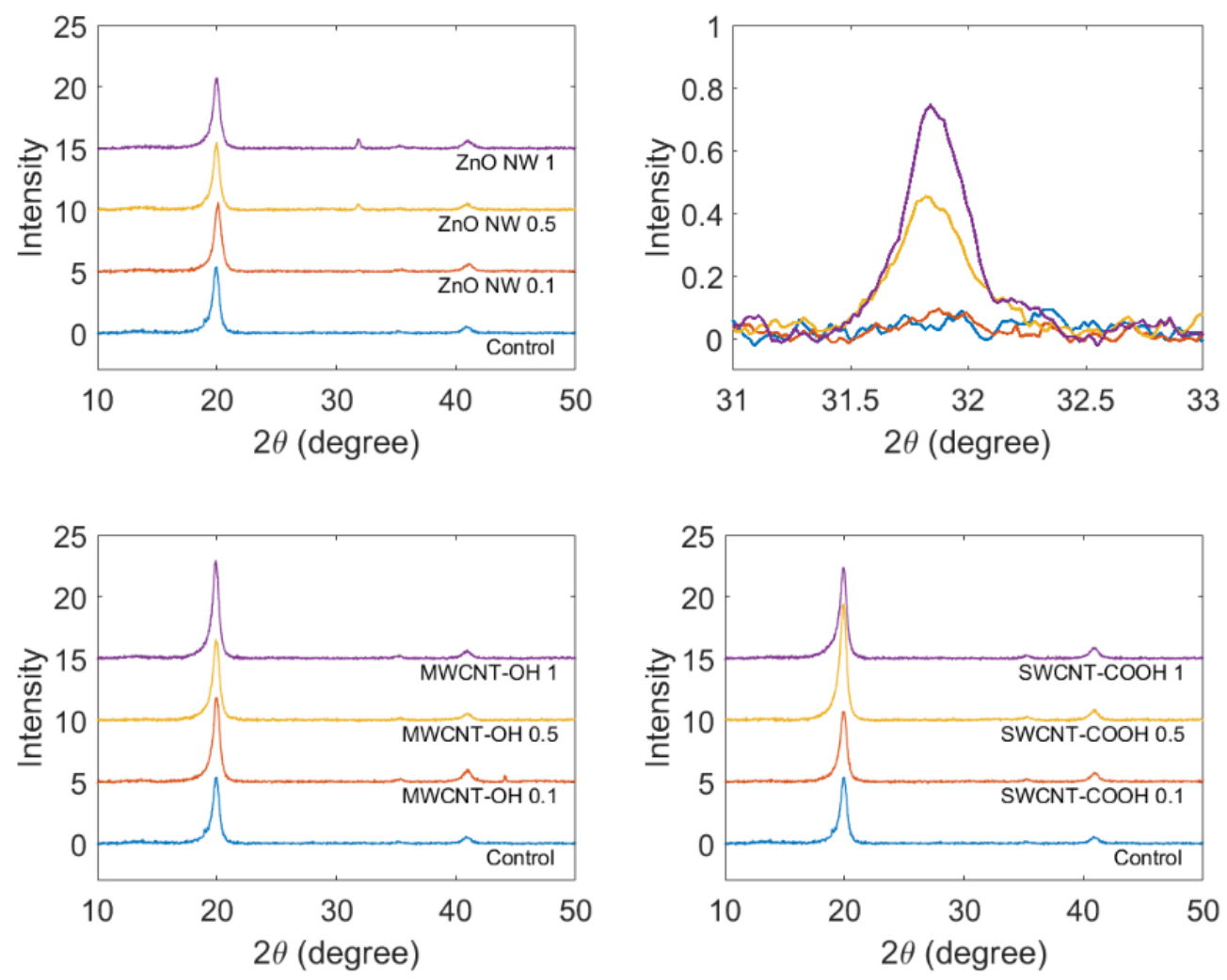

Figure 28. XRD diffractogram of $\mathrm{P}(\mathrm{VDF}-\mathrm{TrFE})$ films with variable loading of nanoparticle filler.

DSC was employed to gain further understanding about the thermodynamic and crystalline properties of the films. This technique is employed to determine the Curie temperature ( $T_{\text {curie) }}$, melting temperature $\left(T_{m}\right)$, and degree of crystallinity $\left(X_{c}\right)$ within the polymer films. Results from $X_{c}$ measurements are shown in Figure 29. For both ZnO NW and MWCNT-OH nanocomposites, the addition of nanofiller leads to a gradual decrease in the degree of crystallinity. On the other hand, the addition of 0.1 and $0.5 \mathrm{wt} \%$ SWCNT-COOH resulted in around a $5 \%$ increase in crystallinity from $55 \%$ in the $\mathrm{P}(\mathrm{VDF}-\mathrm{TrFE})$ control to $60 \%$. The addition of up to 1 wt $\%$ SWCNT-COOH decreased the crystallinity within the film by a difference of $8 \%$. The melting temperatures of the PNCs remained relatively unchanged from nanoparticle addition (Appendix Table A.5) 


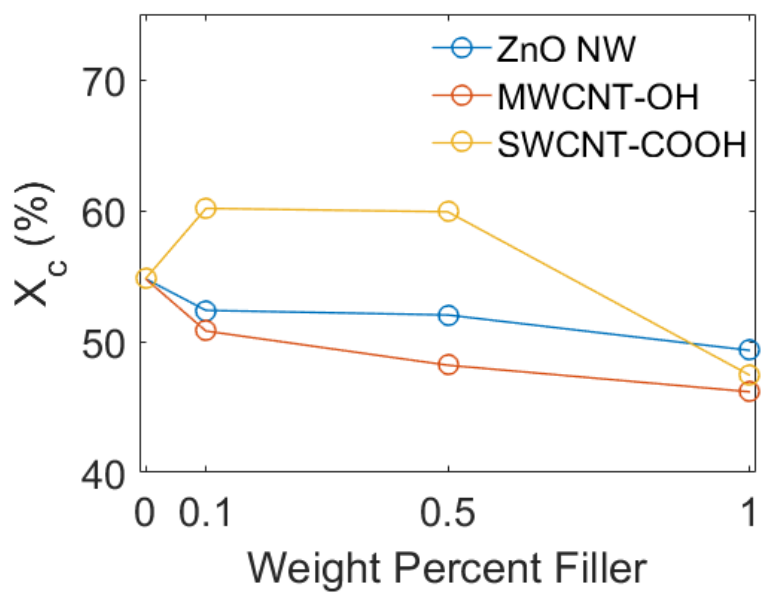

Figure 29. Percent crystallinity $\left(X_{c}\right)$ of polymer nanocomposites with varying loading of $\mathrm{ZnO} N W$, MWCNT-OH, and SWCNT-COOH.

FTIR and XRD measurements showed that addition of nanofiller did not significantly change the large amount of $\beta$-phase in $\mathrm{P}(\mathrm{VDF}-\mathrm{TrFE})$ films. Thus, the relative percentage of crystalline phase within the semi-crystalline matrix is proportional to the overall volume of $\beta$-phase within the film. Results from XRD measurements showed a slight decrease in the degree of crystallinity, and thus a decrease in the volume of present $\beta$-phase relative to amorphous polymer, for all nanocomposites besides $0.1 \mathrm{wt} \%$ and $0.5 \mathrm{wt} \%$ SWCNT-COOH.

In order to further investigate the effect of nanoparticle addition on the microstructure of the PNCs, the surface of dried $100 \mu \mathrm{m}$ cast films were probed using AFM. Previous studies have shown that $\mathrm{P}(\mathrm{VDF}-\mathrm{TrFE})$ films form needle-like microstructures when annealed between the curie temperature and melting temperature as lamellar stacks grow along the long axis direction of the needles. ${ }^{68,70}$ Piezoelectric performance has been suggested to be impacted by the morphology of the crystal structures in previous studies characterizing P(VDF-TrFE) thin films. ${ }^{71}$ Strong piezoelectric performance from these microstructures has been attributed to edge-on crystallization of microdomains which results in polar b-axis orientation normal to the film. ${ }^{72}$

The surface morphology of pure $\mathrm{P}(\mathrm{VDF}-\mathrm{TrFE})$ shows the presence spherical crystalline grains with the diameter of several micrometers. 


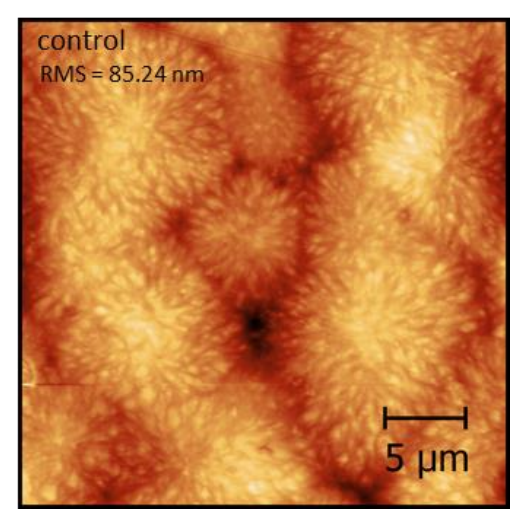

Figure 30. Atomic force microscopy topograph of control P(VDF-TrFE) film with no added filler.

The addition of $\mathrm{ZnO} N W$ as low as $0.1 \mathrm{wt} \%$ seemed to significantly alter the microstructure at the surface of the films. AFM images in Figure 21 show the resulting surface structure of the PNCs after the addition of $0.1,0.5$ and 1 wt $\% \mathrm{ZnO}$ NW. Long, flexible lamellar fibers multiple microns in length are observed randomly oriented in the films. It is possible that the addition of $\mathrm{ZnO}$ NW is disrupting the formation of polymer crystalline macrostructures which is leading to the random orientation of crystalline polymer fibers observed.
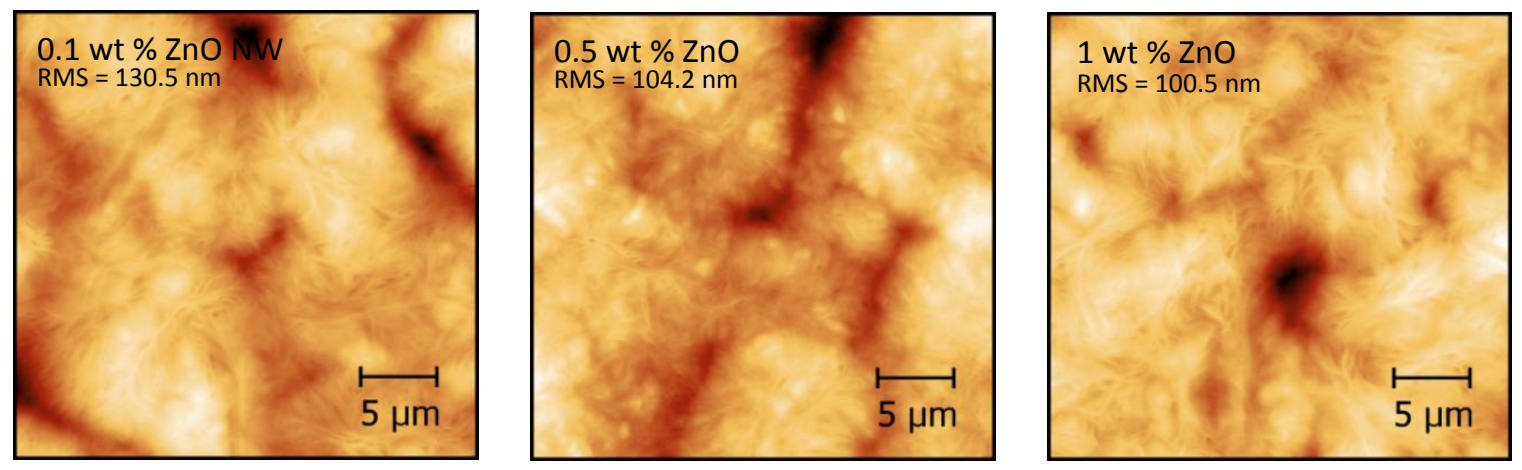

Figure 31. Atomic force microscopy topographs of P(VDF-TrFE) PNCs with (left to right) 0.1 wt \%, 0.5 wt $\%$, and 1 wt $\% \mathrm{ZnO}$ NW.

Unlike that of the pure P(VDF-TrFE) and ZnO nanocomposite films, CNT films show needle-like crystalline structures that are similar to microstructures observed with $\mathrm{P}(\mathrm{VDF}-\mathrm{TrFE})$ thin films $(<100 \mathrm{~nm})$ previously reported. The decrease in microstructure size could be attributed to increased surface nucleation on the nanofiller surfaces, an effect previously reported with PVDF 
homopolymer with the addition of nanofillers. ${ }^{6}$ The decrease in crystallite size in CNT composite samples was also clearly observed through POM imaging, confirming that the decrease in microstructure size exists throughout the sample and not just a localized region imaged with AFM (Appendix Figure A.4). In MWCNT-OH nanocomposites, needle-like microstructures a few microns in length seem to retain the same morphology and size throughout the addition of $0.1,0.5$, and 1 wt \% nanofiller.
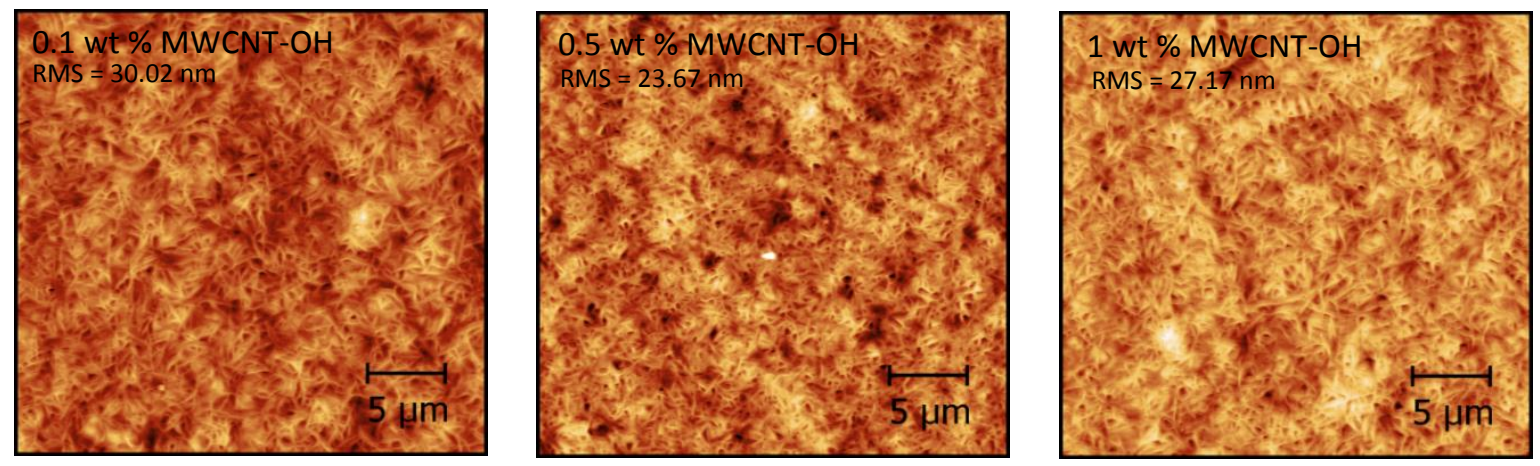

Figure 32. Atomic force microscopy topographs of P(VDF-TrFE) PNCs with (left to right) $0.1 \mathrm{wt} \%$, 0.5 wt $\%$, and 1 wt $\%$ MWCNT-OH.

Films with $0.1 \mathrm{wt} \%$ SWCNT-COOH showed needle-like polymer microstructures similar to those observed in MWCNT-OH samples. As the loading of SWCNT-COOH is increased to 0.5 wt \%, the crystal microstructures significantly decrease in length. At 1 wt \% SWNCT-COOH loading, the needle-like morphology of the polymer is lost, and crystallites becomes too small to distinguish.
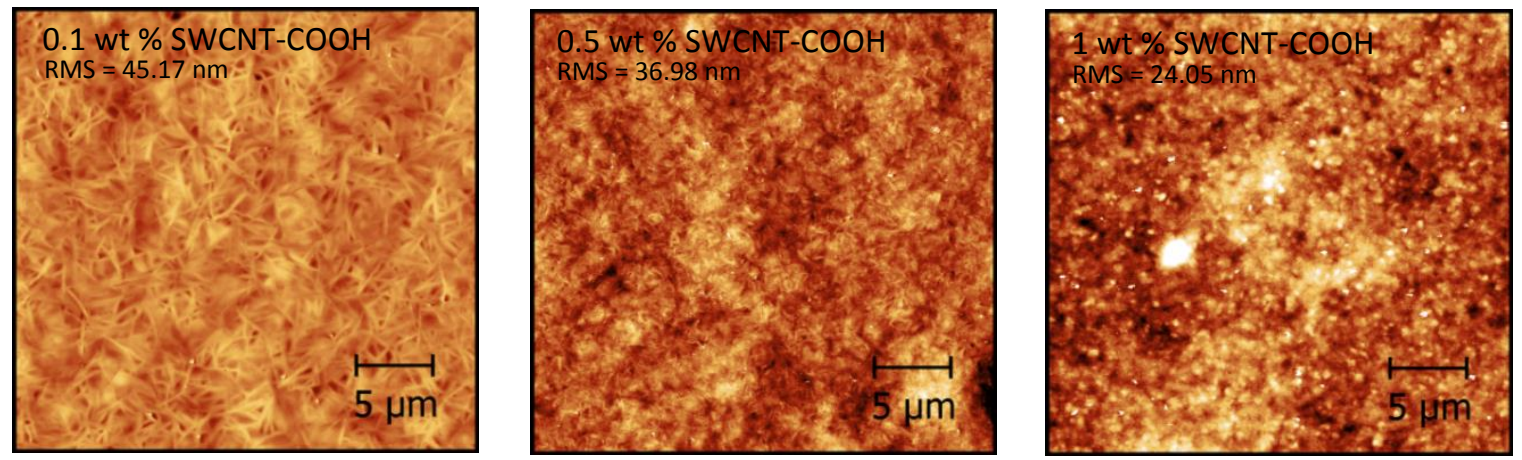

Figure 33. Atomic force microscopy topographs of P(VDF-TrFE) PNCs with (left to right) 0.1 wt \%, 0.5 wt $\%$, and 1 wt $\%$ SWCNT-COOH. 
Properties such as RMS surface roughness are obtained from AFM topographs through image processing software to glean information relating to the surface structure of the nanocomposites. In relation to the control film, an increase in roughness is observed with the addition of $\mathrm{ZnO} \mathrm{NW}$, whereas a significant decrease in roughness is observed when adding either CNT filler. In samples between 0.1 and 1 wt $\%$ MWCNT-OH, there is no significant observed change in RMS. Samples with SWCNT-COOH loading shows a decrease in RMS as nanofiller is added.

The increase in RMS of samples with ZnO NW appears to be due to the formation of peaks and troughs from higher order $(>10 \mu \mathrm{m})$ structures formed from stacked lamellar fibers. Comparatively, samples loaded with CNT do not appear to have these higher order structures, but rather consist of randomly distributed grains. When comparing topographs, it appears that the RMS directly corresponds to grain size in CNT samples.

Table 3. RMS Surface Roughness of Polymer Nanocomposite AFM Topographs

\begin{tabular}{lr}
\hline \multicolumn{1}{c}{ Sample } & RMS Roughness $(\mathrm{nm})$ \\
\hline Control & 85.24 \\
ZnO NW 0.1 & 130.50 \\
ZnO NW 0.5 & 104.20 \\
ZnO NW 1 & 100.50 \\
MWCNT-OH 0.1 & 30.02 \\
MWCNT-OH 0.5 & 23.67 \\
MWCNT-OH 1 & 27.17 \\
SWCNT-COOH 0.1 & 45.17 \\
SWCNT-COOH 0.5 & 36.98 \\
SWCNT-COOH 1 & 24.05 \\
\hline
\end{tabular}




\subsubsection{Power Harvesting}

The output power of the $\mathrm{P}(\mathrm{VDF}-\mathrm{TrFE}) \mathrm{PNG}$ was characterized using the Instron compression tester setup. Results from the test showed that there was a nonlinear increase in output voltage with nanoparticle addition. The addition of ZnO NW demonstrated a slight increase in voltage which maximized at $0.5 \mathrm{wt} \%$ nanofiller addition, but then decreased when the weight loading of the filler was increased to $1 \mathrm{wt} \%$. The addition of MWCNT-OH increased the observed voltage with a loading as low as $0.1 \mathrm{wt} \%$, although little increase was observed from subsequent loading up to $1 \mathrm{wt} \%$ filler. On the other hand, the addition of $0.5 \mathrm{wt} \%$ SWCNT-COOH showed a large jump in output voltage, which seemed to not change much increasing loading to $1 \mathrm{wt} \%$

\section{SWCNT-COOH.}
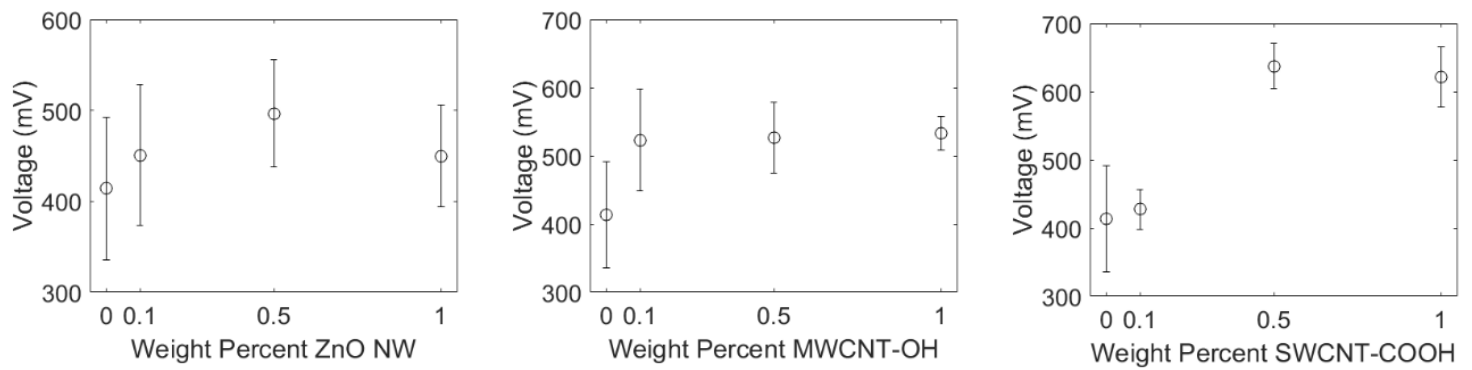

Figure 34. Output voltage of devices from Instron compression testing.

As well, a general increase in device output current from Instron testing was observed with the addition of nanofiller. Parabolic trends are observed in output current with the addition of $\mathrm{ZnO}$ NW and MWCNT-OH, with a peak in current for both filler types at a loading of $0.5 \mathrm{wt} \%$. On the other hand, in SWCNT-COOH formulations, the output current peaks at $0.1 \mathrm{wt} \%$ filler, and is slightly diminished thereon.
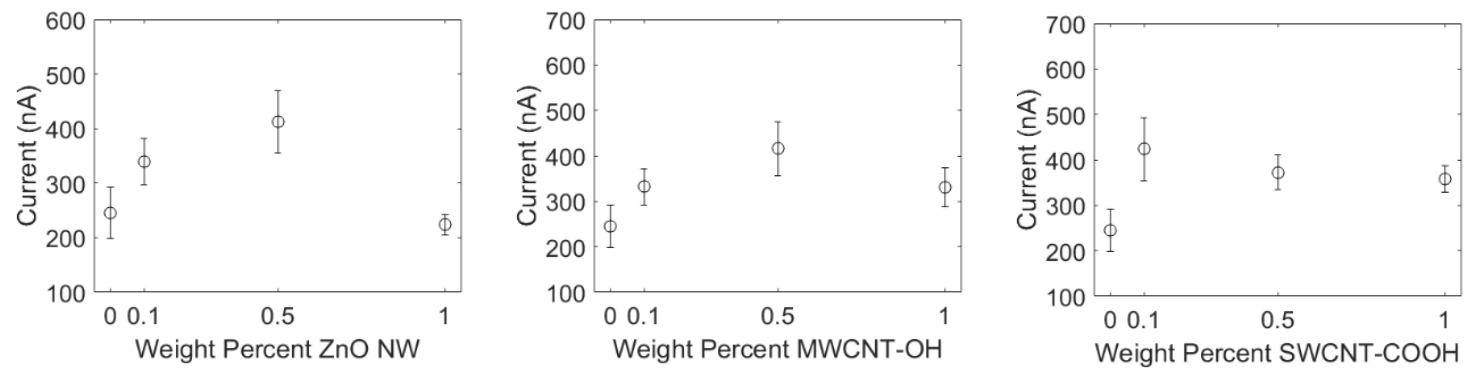

Figure 35. Output current of devices from Instron compression testing. 
The output volume power densities of the devices were calculated using current and voltage data from instron testing and the film volume (assuming an average film thickness of 10 $\mu \mathrm{m})$. A parabolic trend was observed with the addition up to $1 \mathrm{wt} \%$ nanofiller with peak power density observed at $0.5 \mathrm{wt} \%$ filler. Above that, the power density drops off, most notably so with the ZnO NW devices. The addition of SWCNT-COOH showed a considerable increase of power density at all loadings, reaching a maximum of $67.8 \pm 5.2 \mu \mathrm{W} \mathrm{cm}{ }^{-3}$ at $0.5 \mathrm{wt} \%$ loading, more than a twofold increase from the P(VDF-TrFE) film without filler which showed a power density of 29.0 $\pm 5.2 \mu \mathrm{W} \mathrm{cm}-3$. We can calculate the piezoelectric voltage constant using the equation $\left(g_{33}=\frac{E}{\sigma}=\right.$ $\left.\frac{V_{o}}{\sigma t}\right)$ knowing that the average applied force of the Instron was $200 \mathrm{~N}$ and the dimensions of the nanogenerator films. If assume that the recorded voltage from the oscilloscope represents the film's open circuit voltage, the maximum voltage constant from $0.5 \mathrm{wt} \%$ SWCNT-COOH samples is $\mathrm{g}_{33}$ $=0.112 \pm 0.012 \mathrm{Vm} \mathrm{N}^{-1}$. It is important to note that the power density and piezoelectric voltage constant are dependent on film thickness, so a small film thickness significantly increases these values.

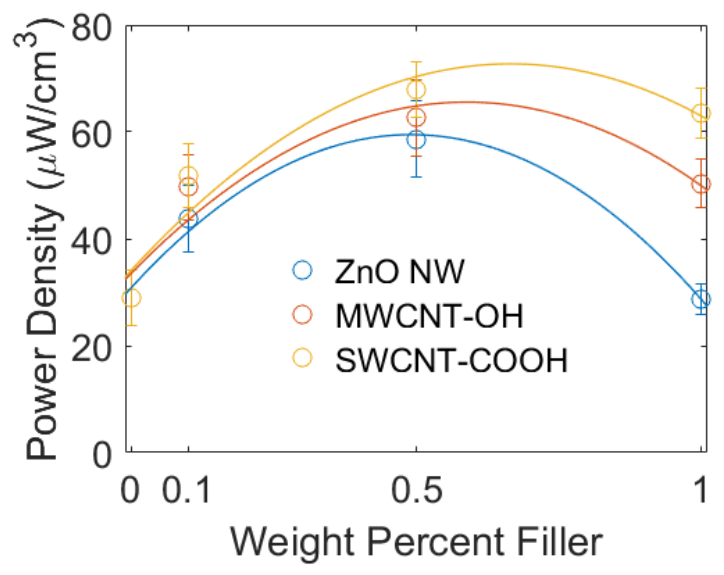

Figure 36. Device volume power density from Instron compression testing. 
The shape of the voltage signal from Instron compression testing was investigated to understand the mechanism behind power generation. A closer look at the signal from Instron compression testing (Figure 37) shows a 74 ms end-to-end signal, with a mirroring decompression peak that shows a similar magnitude of output voltage and signal duration.
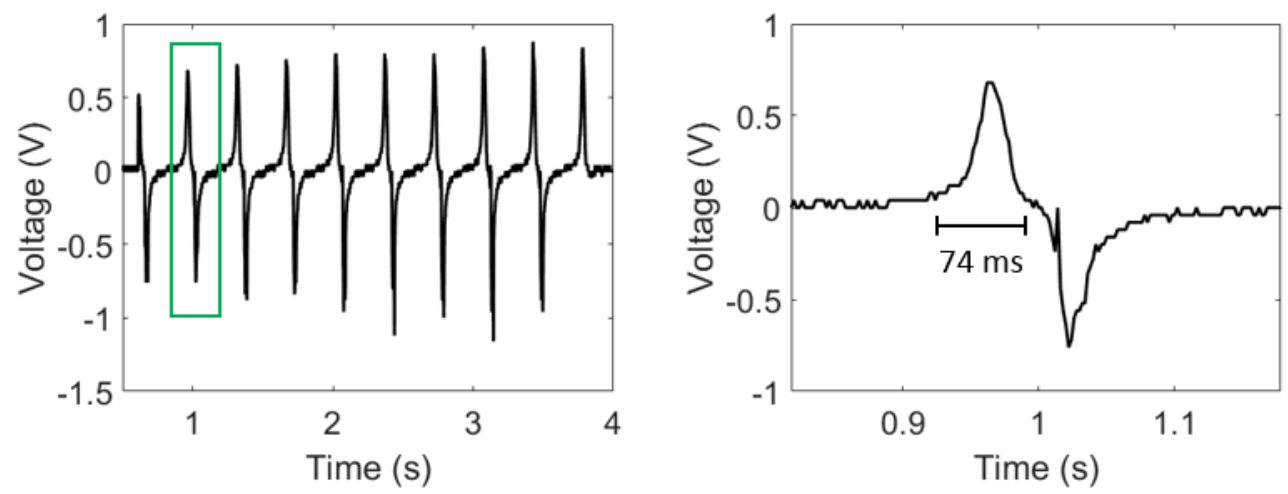

Figure 37. Oscillatory compression test output voltage using 0.5 wt $\%$ SWCNT-COOH film over (left) multiple seconds and (right) a single compression/decompression cycle showing a $74 \mathrm{~ms}$ compressive signal.

In comparison, a simple hand tapping test was employed and output voltages were recorded. Quick tapping pulses imparted onto the film showed considerably higher output voltages (Figure 38), with a peak output voltage $\mathrm{V}_{\max }=13 \mathrm{~V}$. When comparing the signal of this mode of actuation to that of the Instron compression signal, a lower end-to-end signal of $\approx 8 \mathrm{~ms}$ is observed. Also, the decompression signal shows a much lower output voltage than that of the compressive signal, unlike the equal magnitude signals observed in Instron compression testing. It is important to note that throughout all compression tests, it was observed that the speed of PNG film compression had a large effect on their output power. This phenomenon was demonstrated through Instron compression testing with an incremental increase in oscillatory tapping frequency (Appendix A.5). 

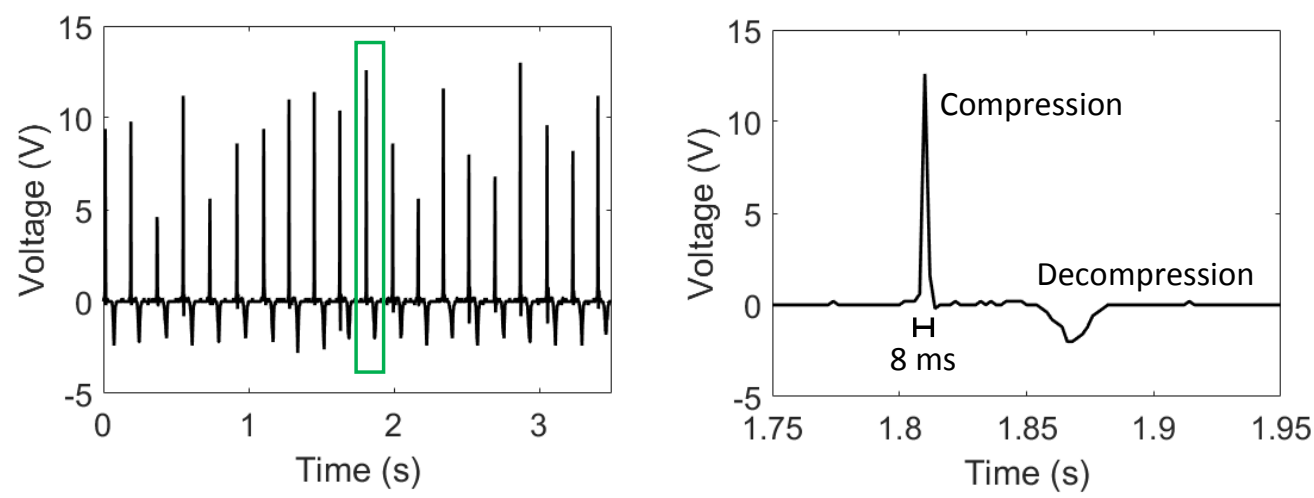

Figure 38. Output voltage from tapping SWCNT-COOH 0.5 (left) tapping over time showing sustained output voltage and (right) peak and trough outlined in left figure demonstrating typical time duration of compressive and decompressive voltage peaks.

The current generated by this tapping mode of deformation was also recorded to gather information about power generation. Observed output current (Figure 39) from tapping showed a peak output current of $I_{\max }=7.8 \mu \mathrm{A}$ and an average output current of $I_{\text {ave }}=4.8 \mu \mathrm{A}$. The shape of these signals is similar to observed voltage signals.

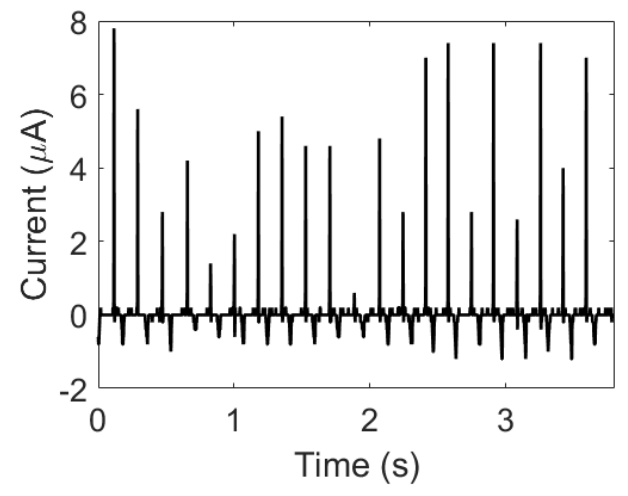

Figure 39. Output current from tapping SWCNT-COOH 0.5 tapping.

From the peak current and voltage of the tapping test, the maximum volume power density was found to be $29 \mathrm{~mW} \mathrm{~cm}^{-3}$, with an average of $12.3 \mathrm{~mW} \mathrm{~cm}^{-3}$. The rectified voltage output from the compressive normal stress of tapping, as seen in Figure 40, leads to the generation of only a positive output voltage. The rectified PNC was used to turn on a red LED directly through compressive tapping (Figure 40b inset). 
(a)

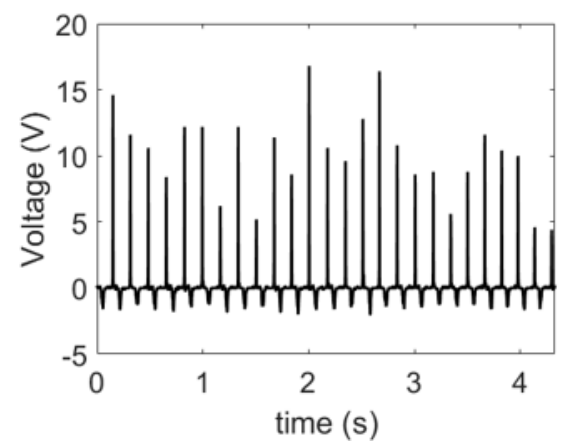

(b)

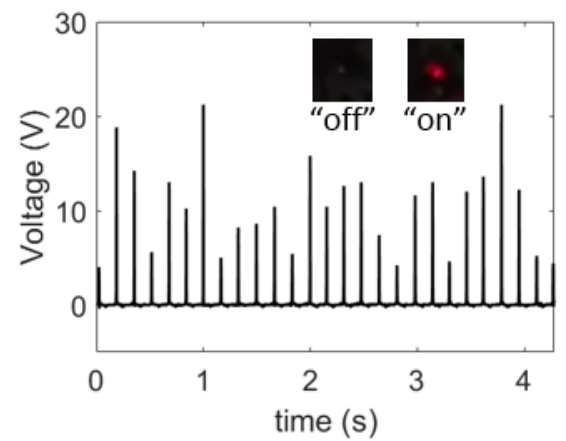

Figure 40. Output voltage from tapping $0.1 \mathrm{wt} \% \mathrm{ZnO}$ NW PNG with (left) no rectification (right) with full wave bridge rectifier.

The energy harvesting capabilities of the film were demonstrated through charging a 10 $\mathrm{nF}$ capacitor using the rectified circuit. The SPDT switch was used to subsequently discharge the capacitor to power a display. With ten seconds of tapping, the capacitor was charged to $29 \mathrm{~V}$ which could be used to power multiple display systems including an array of LED lights (Figure 41).

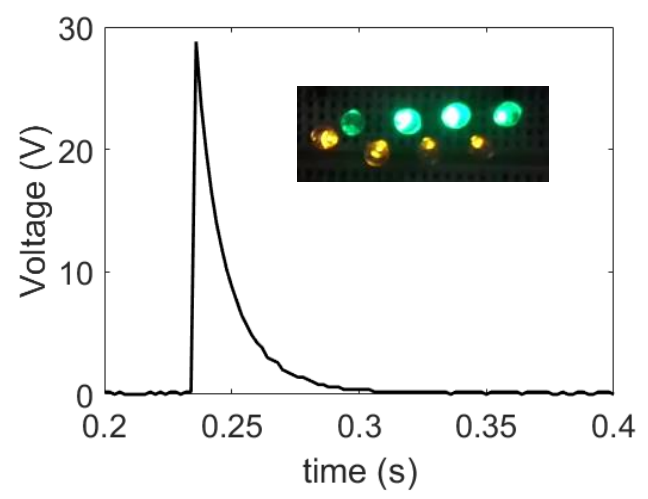

Figure 41. Discharge of $10 \mathrm{nF}$ capacitor charged with 10 seconds moderate hand tapping used to power several green and yellow LED lights (inset).

To demonstrate that the output signals were generated from the piezoelectric effect rather than from the instrumental noise, the switching-polarity test was conducted with piezoelectric films. ${ }^{73}$ This test is passed when the reversal of the sign of compression and decompression voltage peaks is observed after the probes of the measurement device are reversed on the PNG. Results 
from light finger tapping on the $0.5 \mathrm{wt} \% \mathrm{SWCNT}-\mathrm{COOH}$ device (Figure 42) demonstrate the reversal of peak output signal when the leads are reversed. This signal reversal is indicative of the presence of a unidirectional polarization normal to the plane of the PNG film.
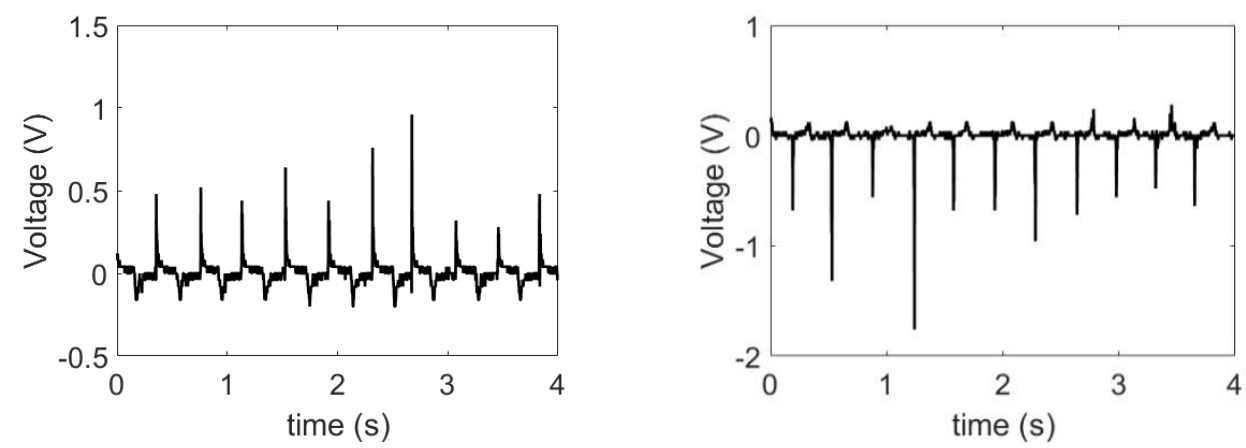

Figure 42. Output voltage in the (left) forward and (right) reverse direction showing polarizationswitching with the $0.5 \mathrm{wt} \%$ SWCNT-COOH film.

Several different modes of film deformation were tested to demonstrate the efficiency of energy harvesting for various possible uses. The first test involved flicking the device by hand while its base was stabilized and immobile. The flicking motion gives two signals: first, from a downwards bending stress imparted by hand, and second, a quick upwards bend due to restoring forces. As a result, two signals are observed from each flicking motion with opposite signs due based on the direction of bending strain. Flicking had a max output voltage of $-7.56 \mathrm{~V}$ from the downward flick with an average voltage of around $-3.5 \mathrm{~V}$. The maximum voltage from the subsequent upward flick was $2.8 \mathrm{~V}$ while the average was around $1 \mathrm{~V}$.

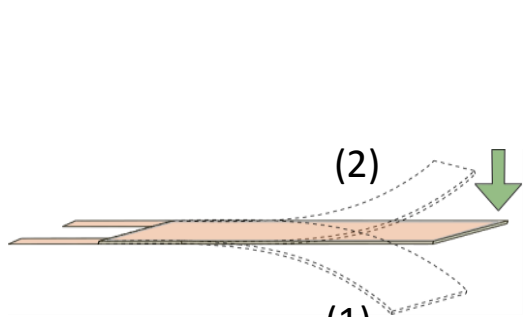

(1)
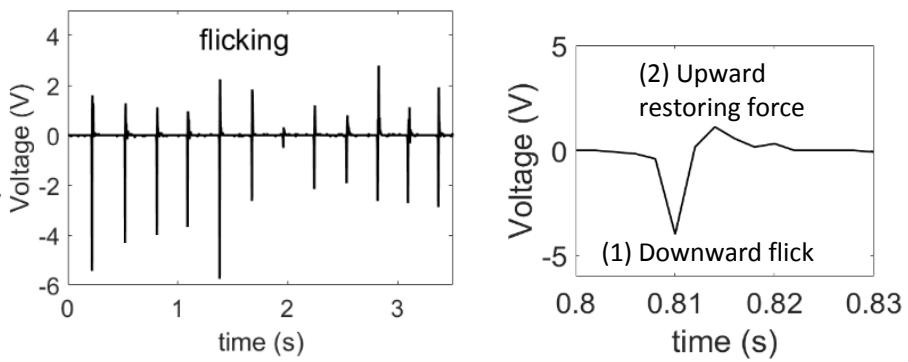

Figure 43. Voltage generation through flicking (left) overview of signals observed (right) single signal. 
The second test involved bending the film at a frequency of $4.8 \mathrm{hz}$. Similar to the flicking test, a signal is produced by the bending stress from film deformation within the polarized semicrystalline film, and thus as signal is produced. The restoring force returns the film to its original unbent shape which causes a movement of polarized crystals within the film to their original form, resulting in a signal with a reversed sign. An average output voltage of about $270 \mathrm{mV}$ and $390 \mathrm{mV}$ was observed from bending and unbending, respectively.

The final mode of film deformation tested involves the application of a ligh compressive "pulse" onto the device without removing hand contact. Similar to tapping, this method imparts pressure normal to the plane of the film which causes a large amount of strain throughout the small distance of the film's thickness. An average output voltage of of $280 \mathrm{mV}$ and $-1.3 \mathrm{mV}$ was observed from this form of compression and decompression, respectively.
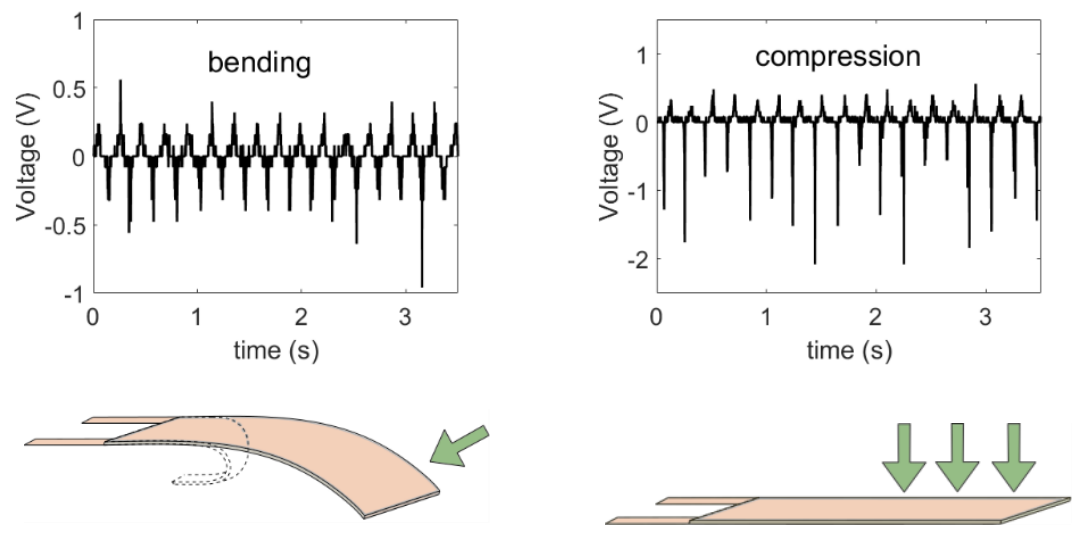

Figure 44. Voltage generation through (left) film bending and (right) compressive force from finger pulsing. 


\section{CONCLUSION}

In summary, two film systems were designed and tested to determine how the piezoelectric properties of electroactive PVDF copolymers are influenced by the addition of various nanomaterials. The introduction of $1 \mathrm{wt} \% \mathrm{MWCNT}-\mathrm{OH}$ into the P(VDF-HFP) resulted in a $26 \%$ increase in the electroactive $\beta$-crystalline phase and a $600 \mathrm{mV}$ increase in output voltage from tapping testing. No significant change in $\beta$ phase or output voltage was observed from the additional loading of ZnO NW. This result sparked further curiosity regarding the influence of filler type on nanoparticle addition. As a result, the continuation of the project focused on comparing how individual nanoparticles influence a piezoelectric film's properties. P(VDF-TrFE) was used as a polymer matrix as this copolymer is known to produce high amounts of electroactive $\beta$-phase directly from solution casting and is known to have strong piezoelectric properties.

To further characterize nanoparticle influence in PNG films, the relative presence electroactive $\beta$-phase crystal along with the overall degree of crystallinity in P(VDF-TrFE) films were characterized by FTIR, XRD, and DSC. Results from FTIR showed that the film's crystalline regiouns mostly formed the electroactive $\beta$-phase. Results from XRD testing solely show diffraction peaks corresponding to the $\beta$-phase, confirming the observations from FTIR testing. Measurements from DSC tests show a slight decrease in the film's overall degree of crystallinity with the addition of most nanoparticles except for an observed increase from the addition of 0.1 and 0.5 wt \% SWCNT-COOH. Only one run was conducted for each PNC formulation, so repeat tests will help better understand the average degree of crystallinity of the PNC films.

Surface mapping with AFM was conducted to understand the effect of nanoparticle loading on the crystal morphology of the films. An observed decrease in crystallite size was observed with the addition of CNT, and a continual decrease in crystallite size was observed as the loading of SWCNT-COOH was increased.

Upon the addition of nanoparticles into the copolymer matrix, a non-linear increase in output power was observed for all types of nanofiller loading. The nanomaterial which displayed the highest output power was from P(VDF-TrFE) samples with SWCNT-COOH, and the highest power was observed at $0.5 \mathrm{wt} \%$ nanoparticle loading. The piezoelectric voltage constant was 
calculated to be $\mathrm{g}_{33}=0.112 \pm 0.012 \mathrm{Vm} \mathrm{N}^{-1}$ from instron compression testing, and peak output volume power density was calculated to be $29 \mathrm{~mW} \mathrm{~cm}^{-3}$ measured from hand tapping. These values are greatly influenced by the thickness of the nanogenerator films, which were only around $10 \mu \mathrm{m}$, which is significantly thinner than PNGs from other studies.

The energy harvesting capabilities of the PNGs were demonstrated through the use of simple circuitry. PNGs rectified using a full bridge rectifier were demonstrated to be able to power an LED directly from tapping, and rectified power that was used to charge a capacitor showed an output voltage of $30 \mathrm{~V}$ after discharging just from just ten seconds of tapping. This stored power was used to power multiple multicolored LEDs. Significant output voltages was also obtained through different modes of film deformation such as flicking, bending, and compression, demonstrating the variety of motions that can be used for energy harvesting.

A few mechanisms are considered to be producing an output power from the PNGs. The first is electretic polarization from high volume heterogeneous interfacial charge trapping at polymer/filler interfaces. The decrease in crystallite size observed from POM and AFM suggested increased interfacial interactions that may be helping to facilitate charge trapping. The formation of smaller crystallites induced in CNT/fluoropolymer nanocomposites has previously been reported to help facilitate higher charge storage within the nanofiller-polymer interface. ${ }^{58}$ Annealing-induced polarization in $\mathrm{P}(\mathrm{VDF}-\mathrm{TrFE})$ between $\mathrm{T}_{\text {curie }}$ and $\mathrm{T}_{\mathrm{m}}$ was previously reported, ${ }^{19}$ and further testing may be run to determine whether this is the case with these prepared PNGs. As well, the triboelectric effect, or the formation of a current from the transfer of charges from one material to another, is considered to be contributor as a small amount of signal was observed when certain materials such as Teflon would make contact with the PNGs. More comprehensive testing is necessary to gain a better understanding of the energy harvesting mechanisms behind the films.

From this study, polymer PNGs with enhanced piezoelectric properties were prepared through the addition of nanoparticle filler without the need for "stretch and poling" processing methods. Results from this study may serve as a framework for further understanding of complex interactions between the nanofiller and piezoelectric polymer matrix. 


\section{FUTURE WORK}

Future tests should focus on obtaining a better understanding on the filler influence in energy harvesting performance based on the results of this study. Mechanical testing using dynamic mechanical analysis (DMA) will determine influence of film stiffness on the mechanical energy transfer for transduction to an electrical signal, a trend that has shown to lead to a significant increase in piezoelectric properties in previous reports. ${ }^{54}$ Also, device testing such as capacitance measurements and output power as a function of load resistance will help further characterize the electrical properties of the film and their influence on energy harvesting.

Along with that, obtaining a better understanding of the energy harvesting mechanisms in these PNGs will be important to further increase their energy harvesting properties. More advanced spectroscopic techniques such as 2-dimensional grazing incidence X-ray diffraction (2D GIXD) can be employed to analyze the three-dimensional crystalline orientation of the lamellae within the film. This test can be used to determine if there is dipole-oriented unidirectional polarization attributed to the preferred edge-on lamellar orientation normal to the plane of the film.

Optimizing the application of screen-printable PEDOT:PSS electrodes will be an important next step to increasing the film's flexibility and reducing any resistive barriers that might be decreasing charge flow. Testing with other electrode designs such as PET/ITO, screen-printable silver ink, or sputtered metal might be of interest to determine the best electrode material. Proper contact between wire leads and electrodes should be made to maintain contact without inducing shorting between electrodes. Developing a proper electrode design will be key to improving the output power of the PNGs. 


\section{Bibliography}

(1) Xu, Y. Ferroelectric materials and their applications.

(2) Lovinger, A. J. Science 1983, 220 (4602), 1115-1121.

(3) Harrison, J. S. 2001.

(4) Sugano, R.; Tashiro, T.; Sekine, T.; Fukuda, K.; Kumaki, D.; Tokito, S. AIP Adv. 2015, 5 (11), 117106.

(5) Hoffman, J. D.; Miller, R. L. Polymer (Guildf). 1997, 38 (13), 3151-3212.

(6) Sencadas, V.; Martins, P.; Pitães, A.; Benelmekki, M.; Gómez Ribelles, J. L.; LancerosMendez, S. Langmuir 2011, 27 (11), 7241-7249.

(7) Wang, Z. L.; Zhu, G.; Yang, Y.; Wang, S.; Pan, C. Mater. Today 2012, 15 (12), 532-543.

(8) Wang, Z. L.; Song, J. Science 2006, 312 (5771), 242-246.

(9) Park, K.-I.; Lee, M.; Liu, Y.; Moon, S.; Hwang, G.-T.; Zhu, G.; Kim, J. E.; Kim, S. O.; Kim, D. K.; Wang, Z. L.; Lee, K. J. Adv. Mater. 2012, 24 (22), 2999-3004.

(10) Hu, Y.; Zhang, Y.; Xu, C.; Zhu, G.; Wang, Z. L. Nano Lett. 2010, 10 (12), 5025-5031.

(11) Jung, J. H.; Lee, M.; Hong, J.-I.; Ding, Y.; Chen, C.-Y.; Chou, L.-J.; Wang, Z. L. ACS Nano 2011, 5 (12), 10041-10046.

(12) Ghosh, S. K.; Sinha, T. K.; Mahanty, B.; Mandal, D. Energy Technol. 2015, 3 (12), 11901197.

(13) Singh, D.; Choudhary, A.; Garg, A. ACS Appl. Mater. Interfaces 2018, 10 (3), 2793-2800.

(14) Karan, S. K.; Bera, R.; Paria, S.; Das, A. K.; Maiti, S.; Maitra, A.; Khatua, B. B. Adv. Energy Mater. 2016, 6 (20), 1601016.

(15) Katzir, S. Archive for History of Exact Sciences. Springer 2003, pp 61-91.

(16) Curie, J.; Curie, P. Comptes Rendus 1881, No. 93, 1137-1140.

(17) Lippmann, G. Ann. Chemie Phys. 1881, 24, 145-178.

(18) APC International. Top Uses of Piezoelectricity in Everyday Applications | APC International Ltd. https://www.americanpiezo.com/blog/top-uses-of-piezoelectricity-ineveryday-applications/ (accessed Oct 13, 2018).

(19) Ducrot, P.-H.; Dufour, I.; Ayela, C. Sci. Rep. 2016, 6 (1), 19426.

(20) Lucibella, M.; Voss, D. March 1880: The Curie Brothers Discover Piezoelectricity https://www.aps.org/publications/apsnews/201403/physicshistory.cfm (accessed Nov 17, 2018).

(21) Hehn, T.; Manoli, Y. CMOS circuits for piezoelectric energy harvesters : efficient power extraction, interface modeling and loss analysis.

(22) Uchino, K. Introduction to Piezoelectric Actuators and Transducers.

(23) Jaffe, B.; Cook, W. R.; Jaffe, H. L. Piezoelectric ceramics; Academic Press, 1971.

(24) Briscoe, J.; Jalali, N.; Woolliams, P.; Stewart, M.; Weaver, P. M.; Cain, M.; Dunn, S. Energy Environ. Sci. 2013, 6 (10), 3035. 
(25) Moheimani, S. O. R.; Fleming, A. J. Piezoelectric Transducers for Vibration Control and Damping; Advances in Industrial Control; Springer-Verlag: London, 2006.

(26) Das, R.; He, X.; Ghaffarzadeh, K. Flexible, Printed and Organic Electronics 2019-2029: Forecasts, Players \&amp; Opportunities; 2018.

(27) Zhang, S. Conductive Polymers.

(28) Fukada, E. ieee Trans. Ultrason. Ferroelectr. Freq. Control 2000, 47 (6).

(29) Fukada, E.; Eguchi, M. Phil. Mag 1925, 49 (289), 178-192.

(30) Brown, L. F.; Harris, G. R. IEEE Trans. Ultrason. Ferroelectr. Freq. Control 2000, 47 (6), 1275-1276.

(31) Zhang, S. 2017.

(32) Künstler, W.; Wegener, M.; Seiß, M.; Gerhard-Multhaupt, R. Appl. Phys. A Mater. Sci. Process. 2001, 73 (5), 641-645.

(33) Personick, M. L.; Mirkin, C. A. J. Am. Chem. Soc. 2013, 135 (49), 18238-18247.

(34) Sun, Y.; Xia, Y. Science 2002, 298 (5601), 2176-2179.

(35) Suresh, S. Nanosci. Nanotechnol. 2013, 3 (3), 62-74.

(36) Kumar, S. K.; Benicewicz, B. C.; Vaia, R. A.; Winey, K. I. Macromolecules 2017, 50 (3), 714-731.

(37) Kojima, Y.; Usuki, A.; Kawasumi, M.; Okada, A.; Fukushima, Y.; Kurauchi, T.; Kamigaito, O. J. Mater. Res. 1993, 8 (05), 1185-1189.

(38) Burrows, N. D.; Vartanian, A. M.; Abadeer, N. S.; Grzincic, E. M.; Jacob, L. M.; Lin, W.; Li, J.; Dennison, J. M.; Hinman, J. G.; Murphy, C. J. J. Phys. Chem. Lett. 2016, 7 (4), 632641.

(39) Ye, H.-J.; Shao, W.-Z.; Zhen, L. J. Appl. Polym. Sci. 2013, 129 (5), 2940-2949.

(40) Kim, G. H.; Hong, S. M.; Seo, Y. Phys. Chem. Chem. Phys. 2009, 11 (44), 10506.

(41) Yuan, D.; Li, Z.; Thitsartarn, W.; Fan, X.; Sun, J.; Li, H.; He, C. J. Mater. Chem. C 2015, 3 (15), 3708-3713.

(42) Parangusan, H.; Ponnamma, D.; Al Ali AlMaadeed, M. RSC Adv. 2017, 7 (79), 5015650165 .

(43) Cebe, P.; Runt, J. Polymer (Guildf). 2004, 45 (6), 1923-1932.

(44) Paik, H.; Choi, Y.-Y.; Hong, S.; No, K. Sci. Rep. 2015, 5 (1), 13209.

(45) Zhang, S.; Majewski, P. W.; Keskar, G.; Pfefferle, L. D.; Osuji, C. O. Langmuir 2011, 27 (18), 11616-11621.

(46) Zhang, Y.; Ram, M. K.; Stefanakos, E. K.; Goswami, D. Y. J. Nanomater. 2012, 2012, 122.

(47) Spitalsky, Z.; Tasis, D.; Papagelis, K.; Galiotis, C. Prog. Polym. Sci. 2010, 35 (3), 357401.

(48) Li, Z.; de Barros, A. L. B.; Soares, D. C. F.; Moss, S. N.; Alisaraie, L. Int. J. Pharm. 2017, $524(1-2), 41-54$.

(49) Peng, Y.; Le, Z.; Wen, M.; Zhang, D.; Chen, Z.; Bin Wu, H.; Lu, Y. Nano Energy 2017. 
(50) Roy, S.; David-Pur, M.; Hanein, Y. Carbon N. Y. 2017, 116, 40-49.

(51) Zhang, S. Solubility and Nanotubes; California Polytechnic State University, San Luis Obispo, 2017.

(52) Choudhary, V.; Gupta, A. In Carbon Nanotubes - Polymer Nanocomposites; InTech, 2011.

(53) Park, K.-I.; Lee, M.; Liu, Y.; Moon, S.; Hwang, G.-T.; Zhu, G.; Kim, J. E.; Kim, S. O.; Kim, D. K.; Wang, Z. L.; Lee, K. J. Adv. Mater. 2012, 24 (22), 2999-3004.

(54) Ramaratnam, A.; Jalili, N. J. Intell. Mater. Syst. Struct. 2006, 17 (3), 199-208.

(55) Kim, J.; Loh, K. J.; Lynch, J. P. Tomizuka, M., Ed.; 2008; p 693232.

(56) Luchaninov, A. G.; Shil'nikov, A. V.; Shuvalov, L. A.; Malyshev, V. A. Ferroelectrics 1993, $145(1), 235-239$.

(57) Lee, K. Y.; Kim, D.; Lee, J.-H.; Kim, T. Y.; Gupta, M. K.; Kim, S.-W. Adv. Funct. Mater. 2014, 24 (1), 37-43.

(58) Baur, C.; DiMaio, J. R.; McAllister, E.; Hossini, R.; Wagener, E.; Ballato, J.; Priya, S.; Ballato, A.; Smith, D. W. J. Appl. Phys. 2012, 112 (12), 124104.

(59) Yousefi, N.; Sun, X.; Lin, X.; Shen, X.; Jia, J.; Zhang, B.; Tang, B.; Chan, M.; Kim, J.-K. Adv. Mater. 2014, 26 (31), 5480-5487.

(60) Kodama, H.; Takahashi, Y.; Furukawa, T. Ferroelectrics 1997, 203 (1), 433-455.

(61) Schneider, C. A.; Rasband, W. S.; Eliceiri, K. W. Nat. Methods 2012, 9 (7), 671-675.

(62) Lanceros-Méndez, S.; Mano, J. F.; Costa, A. M.; Schmidt, V. H. J. Macromol. Sci. Part B 2001, 40 (3-4), 517-527.

(63) Salimi, A.; Yousefi, A. A. Polym. Test. 2003, 22 (6), 699-704.

(64) Kim, J.; Lee, J. H.; Ryu, H.; Lee, J.-H.; Khan, U.; Kim, H.; Kwak, S. S.; Kim, S.-W. Adv. Funct. Mater. 2017, 27 (22), 1700702.

(65) Nečas, D.; Klapetek, P. Open Phys. 2012, 10 (1), 181-188.

(66) Piezo Film Sensors Technical Manual; Norristown.

(67) Persano, L.; Dagdeviren, C.; Su, Y.; Zhang, Y.; Girardo, S.; Pisignano, D.; Huang, Y.; Rogers, J. A. Nat. Commun. 2013, 4 (1), 1633.

(68) Kim, J.-H.; Khang, D.-Y. Eur. Polym. J. 2014, 59, 78-83.

(69) Egusa, S.; Wang, Z.; Chocat, N.; Ruff, Z. M.; Stolyarov, A. M.; Shemuly, D.; Sorin, F.; Rakich, P. T.; Joannopoulos, J. D.; Fink, Y. Nat. Mater. 2010, 9 (8), 643-648.

(70) Hahm, S.-W.; Khang, D.-Y. Soft Matter 2010, 6 (22), 5802.

(71) Hahm, S.-W.; Kim, D.; Khang, D.-Y. Polymer (Guildf). 2014, 55 (1), 175-181.

(72) Park, Y. J.; Kang, S. J.; Park, C.; Kim, K. J.; Lee, H. S.; Lee, M. S.; Chung, U.-I.; Park, I. J. Appl. Phys. Lett. 2006, 88 (24), 242908.

(73) Yang, R.; Qin, Y.; Li, C.; Dai, L.; Wang, Z. L. Appl. Phys. Lett. 2009, 94 (2), 022905. 


\section{APPENDIX}

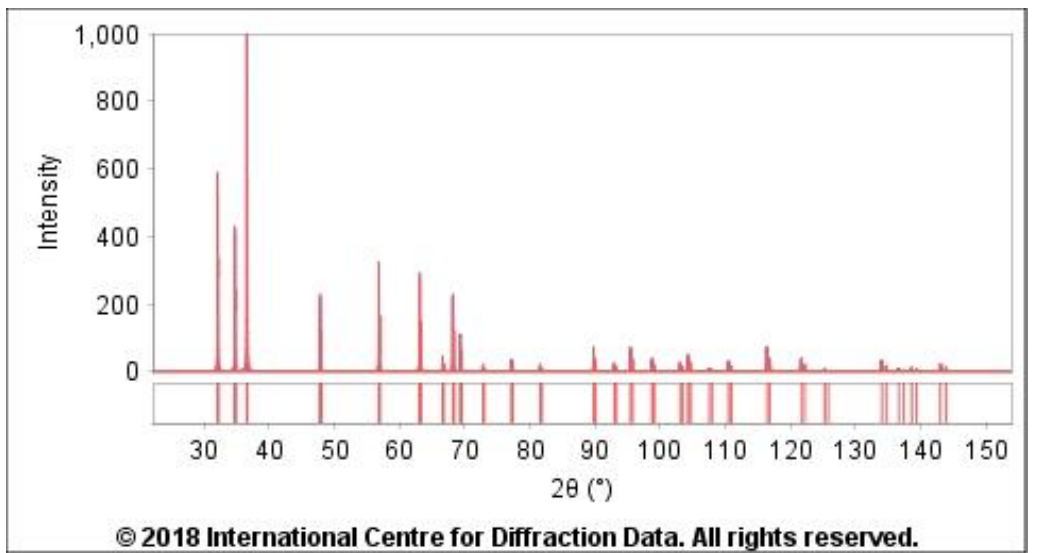

Figure A.1 Reference spectrum PDF Card - 01-070-8070 XRD pattern of hexagonal wurtzite zinc oxide (International Centre for Diffraction Data).

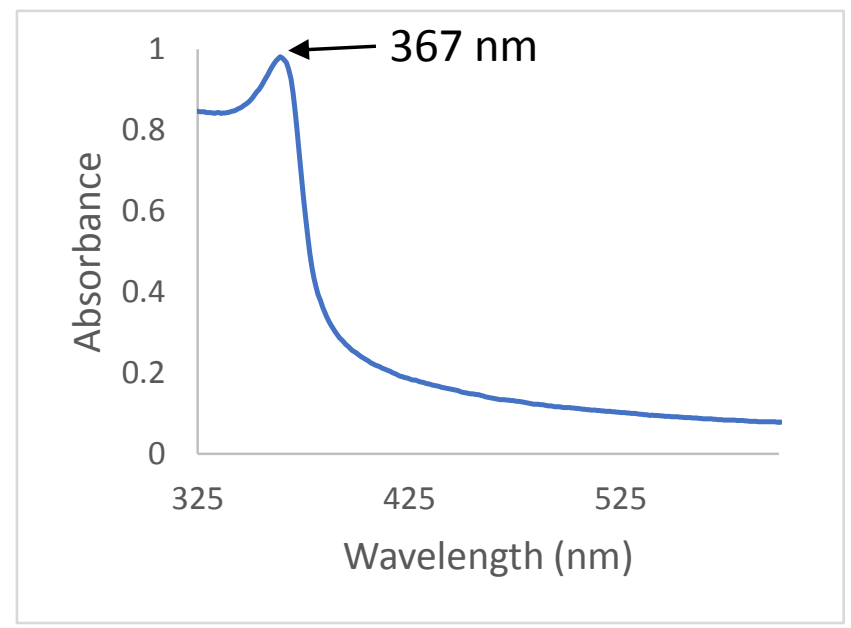

Figure A.2 UV-Vis spectra of ZnO NW with peak at $367 \mathrm{~nm}$ corresponding to $3.37 \mathrm{~V}$ bandgap.

Table A.1 ZnO NW Size Distribution

\section{Average Diameter $(\mathrm{nm}) \quad$ Average Length $(\mathrm{nm}) \quad$ Average Aspect Ratio}

$26.5 \pm 7.0$

$1245 \pm 725$

47 


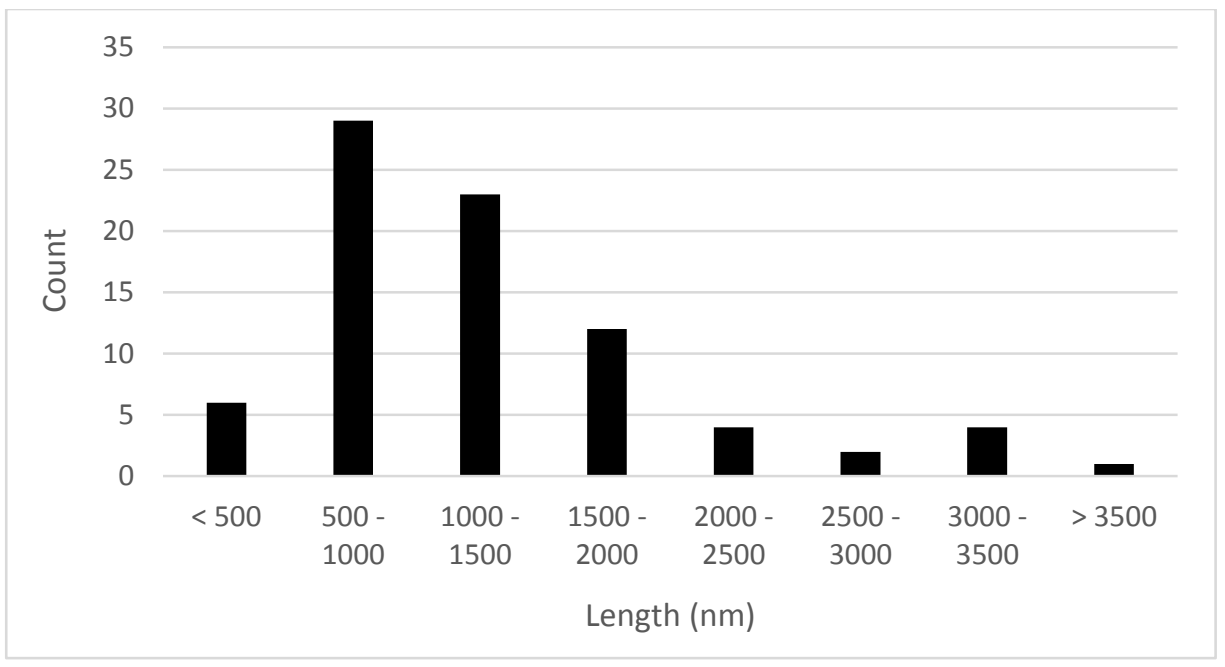

Figure A. 3 Length distribution of synthesized $\mathrm{ZnO}$ NW.

Table A.2 Beta Phase Fraction of P(VDF-HFP) Films Prepared by Blade Casting

\begin{tabular}{|l|r|r|r|}
\hline & 0 wt \% ZnO NW & 4 wt \% ZnO NW & 8 wt \% ZnO NW \\
\hline $100 \mu \mathrm{m}$ wet cast & $0.82 \pm 0.01$ & $0.80 \pm 0.01$ & $0.79 \pm 0.01$ \\
\hline $200 \mu \mathrm{m}$ wet cast & $0.78 \pm 0.03$ & $0.81 \pm 0.01$ & $0.81 \pm 0.03$ \\
\hline
\end{tabular}

Table A.3 Dried Film Thickness Measurements of $100 \mu \mathrm{m}$ Wet Cast Films

\begin{tabular}{|c|c|c|c|}
\hline sample & Average $(\mu \mathrm{m})$ & $\mathrm{SE}(\mu \mathrm{m})$ & count \\
\hline $100 \mu \mathrm{m}$ control & 8.84 & 0.94 & 25 \\
\hline $100 \mu \mathrm{m} \mathrm{ZnO} 0.1$ & 10.2 & 1.2 & 38 \\
\hline $100 \mu \mathrm{m} \mathrm{ZnO} 0.5$ & 13.0 & 1.9 & 23 \\
\hline $100 \mu \mathrm{m} \mathrm{ZnO} 1$ & 10.6 & 2.1 & 7 \\
\hline $100 \mu \mathrm{m}$ MWCNT-OH 0.1 & 11.1 & 1.8 & 21 \\
\hline $100 \mu \mathrm{m}$ MWCNT-OH 0.5 & 11.4 & 1.3 & 26 \\
\hline $100 \mu \mathrm{m}$ MWCNT-OH 1 & 13.41 & 0.61 & 32 \\
\hline $100 \mu \mathrm{m}$ SWCNT-COOH 0.1 & 9.50 & 0.59 & 30 \\
\hline $100 \mu \mathrm{m}$ SWCNT-COOH 0.5 & 10.60 & 0.62 & 30 \\
\hline $100 \mu \mathrm{m}$ SWCNT-COOH 1 & 11.41 & 0.64 & 29 \\
\hline
\end{tabular}


Table A.4 Dried Film Thickness Measurements of $200 \mu \mathrm{m}$ Wet Cast Films

\begin{tabular}{|l|rrr|}
\hline \multicolumn{1}{|c|}{ sample } & Average $(\boldsymbol{\mu m})$ & SE $(\mu \mathrm{m})$ & count \\
\hline $\mathbf{2 0 0}$ um control & 14.7 & 1.3 & 31 \\
$\mathbf{2 0 0}$ um ZnO 0.1 & 14.64 & 0.79 & 31 \\
$\mathbf{2 0 0}$ um ZnO 0.5 & 15.4 & 1.5 & 28 \\
$\mathbf{2 0 0}$ um ZnO 1 & 16.8 & 1.8 & 26 \\
$\mathbf{2 0 0}$ um MWCNT 0.1 & 15.8 & 2.8 & 42 \\
$\mathbf{2 0 0}$ um MWCNT 0.5 & 15.9 & 2.2 & 34 \\
$\mathbf{2 0 0}$ um MWCNT 1 & 20.1 & 1.1 & 30 \\
$\mathbf{2 0 0}$ um SWCNT-COOH 0.1 & 15.4 & 1.1 & 29 \\
$\mathbf{2 0 0}$ um SWCNT-COOH 0.5 & 16.27 & 0.89 & 30 \\
$\mathbf{2 0 0}$ um SWCNT-COOH 1 & 18.97 & 0.83 & 30 \\
\hline
\end{tabular}

Table A.5 Curie Temperature ( $\left.T_{\text {curie }}\right)$, Melting Temperature $\left(T_{m}\right)$, and Degree of Crystallinity $\left(X_{c}\right)$ of Dried $100 \mu \mathrm{m}$ Wet Cast P(VDF-TrFE) Films (2nd ramp)

\begin{tabular}{|l|rrr|}
\hline \multicolumn{1}{|c|}{ Sample } & $\mathrm{T}_{\text {curie }}\left(\mathrm{C}^{\circ}\right)$ & $\mathrm{T}_{\mathrm{m}}\left(\mathrm{C}^{\circ}\right)$ & $\mathrm{X}_{\mathrm{c}}(\%)$ \\
\hline Control & 116.2 & 144.9 & 54.8 \\
0.1 wt \% ZnO NW & 114.0 & 144.0 & 52.4 \\
0.5 wt \% ZnO NW & 114.4 & 144.6 & 52.0 \\
1 wt \% ZnO NW & 114.7 & 145 & 49.3 \\
0.1 wt \% MWCNT-OH & 114.0 & 145.2 & 50.8 \\
0.5 wt \% MWCNT-OH & 113.7 & 144.7 & 48.2 \\
1 wt \% MWCNT-OH & 114.6 & 144.4 & 46.2 \\
0.1 wt \% SWNCT-COOH & 113.4 & 144.6 & 60.2 \\
0.5 wt \% SWCNT-COOH & 113.8 & 144.6 & 59.9 \\
1 wt \% SWCNT-COOH & 113.6 & 144.8 & 47.4 \\
\hline
\end{tabular}



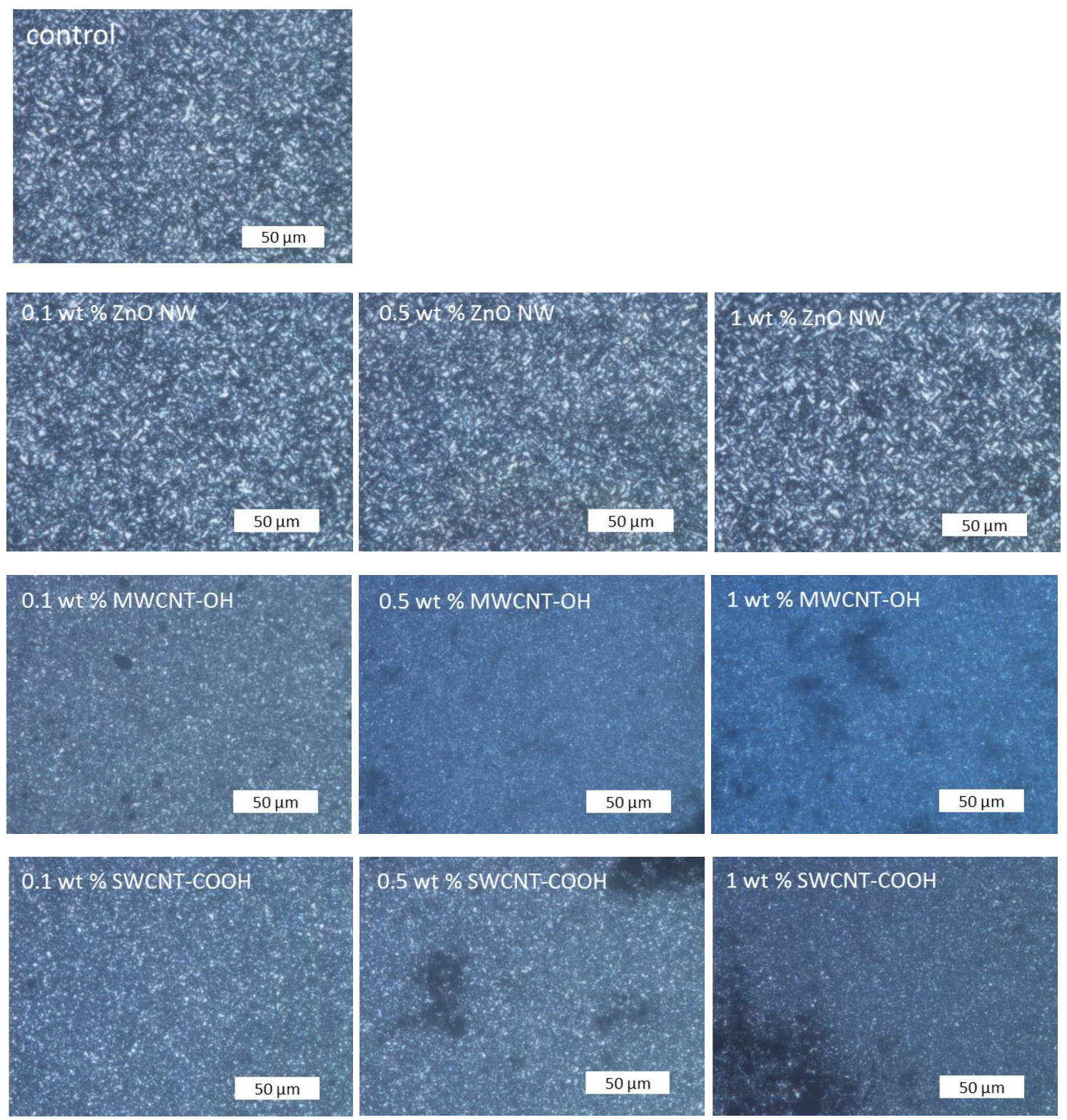

Figure A.4 POM images of dried $100 \mu \mathrm{m}$ wet cast films with varying loading of nanofiller between crossed polarizers. 


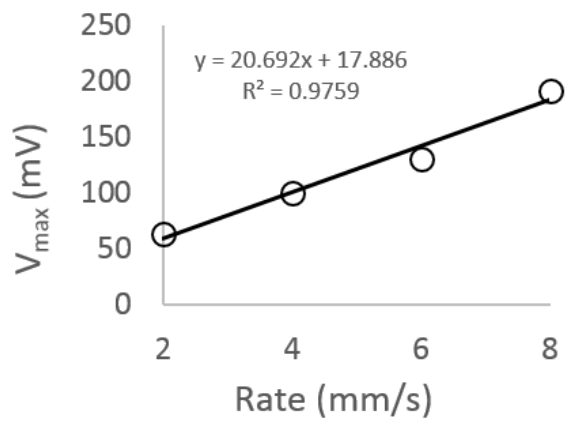

Figure A.5 Peak voltage from Instron compression testing as a function of oscillation rate from direct compression.
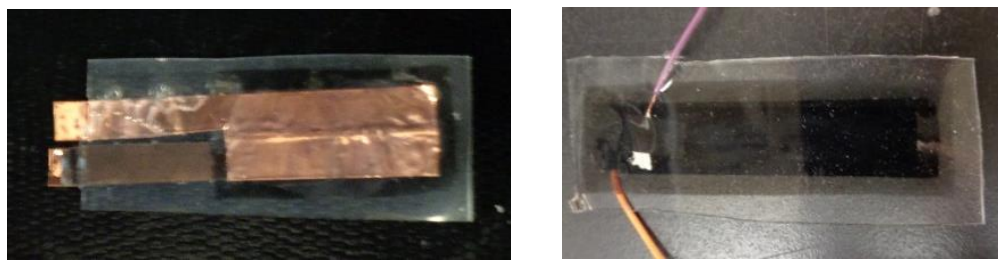

Figure A.7 Device designs with (left) copper tape electrodes and (right) screen printed PEDOT:PSS electrodes. Both devices are encapsulated in PDMS. 